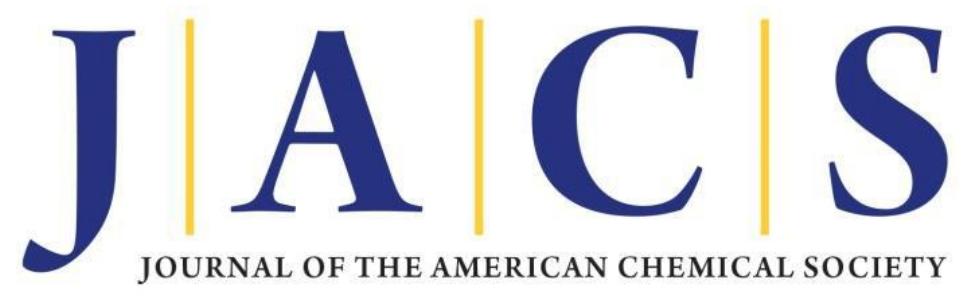

\title{
Sliding-Ring Catenanes
}

Isurika R. Fernando, ${ }^{\dagger}$ Marco Frasconi, ${ }^{\dagger} \S$ Yilei Wu, ${ }^{\dagger, \dagger}$ Wei-Guang Liu, ${ }^{\perp}$ Michael R. Wasielewski, ${ }^{\dagger, \dagger}$ William A. Goddard III, ${ }^{\perp, \#}$ J. Fraser Stoddart* ${ }^{\dagger}$

${ }^{\dagger}$ Department of Chemistry, Northwestern University, 2145 Sheridan Road, Evanston, Illinois 60208, USA

${ }^{\S}$ Department of Chemical Sciences, University of Padova, Via Marzolo 1, Padova 35131, Italy

*Argonne-Northwestern Solar Energy Research (ANSER) Center, Northwestern University, 2145 Sheridan Road, Evanston, Illinois 60208, USA

${ }^{\perp}$ Materials and Process Simulation Center, California Institute of Technology, Pasadena, California 91125, USA

${ }^{\#}$ NanoCentury KAIST Institute and Graduate School of EEWS (WCU), Korea Advanced Institute of Science and Technology (KAIST), 373-1 Guseong Dong, Yuseong Gu, Daejeon 305-701, Republic of Korea

*e-mail: stoddart@northwestern.edu

\section{SUPPORTING INFORMATION}

\section{Table of Contents}

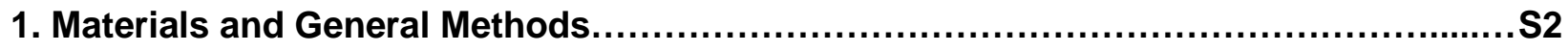

2. Solution-State Characterization of $[2]$ Catenanes............................................... S3

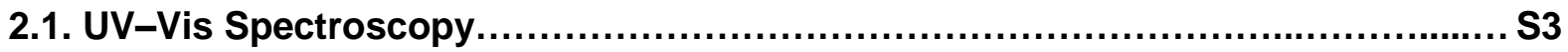

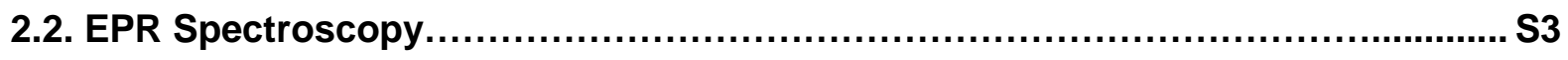

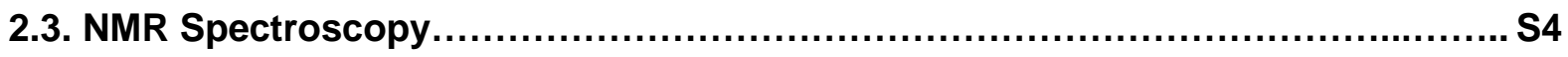

3. Solid-State Characterization of [2]Catenanes...................................................S10

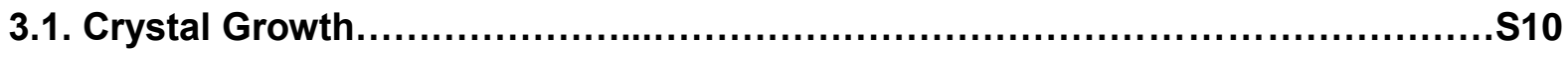

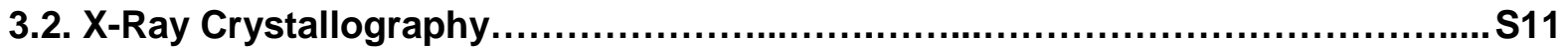

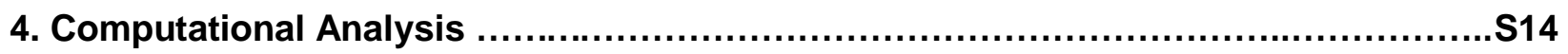

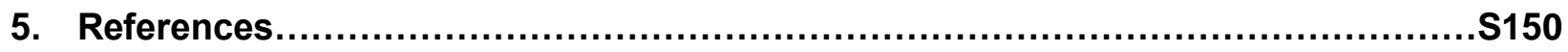




\section{Materials and General Methods}

All reagents were used as received from chemical suppliers (Aldrich and VWR) and reactions were carried out in anhydrous solvents under an inert nitrogen atmosphere, unless otherwise stated. Cyclobis(paraquat- $p$-phenylene) tetrakis(hexafluorophosphate $)^{\mathrm{S} 1} \quad\left(\mathrm{CBPQT} \bullet 4 \mathrm{PF}_{6}\right)$, bisparaphenylene[34]crown-10 ${ }^{\mathrm{S} 2}$ (BPP34C10), 1,5-dinaphtho[38]crown-10 ${ }^{\mathrm{S} 3}$ (DNP38C10), and the catenanes $[\mathrm{DNP} 38 \mathrm{C} 10 / \mathrm{CBPQT}] \cdot 4 \mathrm{PF}_{6}{ }^{\mathrm{S}}\left(\mathbf{1}^{\circ} 4 \mathrm{PF}_{6}\right)$ and $[\mathrm{BPP} 34 \mathrm{C} 10 / \mathrm{CBPQT}] \cdot 4 \mathrm{PF}_{6}{ }^{\mathrm{S}}{ }^{\mathrm{Sb}, \mathrm{S} 5}$ $\left(2 \cdot 4 \mathrm{PF}_{6}\right)$ were prepared according to previously reported literature procedures with minor modifications. Thin layer chromatography (TLC) was performed on Sigma gel 60 F254 TLC plates (Merck) and chromatograms were viewed under UV light (256 nm and / or $354 \mathrm{~nm}$ wavelengths). Column chromatography was carried out on silica gel 60F (Merck 9385, 0.040$0.063 \mathrm{~mm}$ ). UV-Vis Spectra were obtained on a UV-3600 Shimadzu spectrophotometer. ${ }^{1} \mathrm{H}$, ${ }^{13} \mathrm{C}$, ROESY, DOSY and Variable Temperature (VT) Nuclear Magnetic Resonance spectra (NMR) were recorded on a Bruker Avance III spectrometer operating at $600 \mathrm{MHz}$ using deuterated solvents. The temperature $(T)$ was calibrated using neat $\mathrm{MeOH}$ for $T<295 \mathrm{~K}$ and ethylene glycol for $T>295 \mathrm{~K}$ for VT-NMR. Chemical shifts $(\delta)$ were reported in ppm relative to signals corresponding to the residual non-deuterated solvents $\left(\mathrm{C}_{6} \mathrm{D}_{5} \mathrm{CD}_{3}: \delta 2.09 \mathrm{ppm}\right.$ and $\left.\mathrm{CD}_{3} \mathrm{CN}: \delta 1.94 \mathrm{ppm}\right)$. High resolution mass spectra (HRMS) were recorded on an Agilent 6210 Time of Flight (TOF) LC-MS, employing an electrospray ionization (ESI) source, coupled with an Agilent 1100 HPLC stack using direct infusion $\left(0.6 \mathrm{~mL} \mathrm{~min}^{-1}\right)$. Electron Paramagnetic Resonance (EPR) measurements were performed at room temperature using a Bruker Elexsys E580 X-band $(9.5 \mathrm{GHz})$ EPR spectrometer, outfitted with variable Q split ring resonator (ER4118X-MS5-W1). All samples employed in NMR, UV-Vis and EPR experiments were prepared inside an Ar-filled glovebox and the measurements were carried out immediately after sample preparations. Solvents were degassed by purging them with Ar gas during $2 \mathrm{~h}$ prior to transferring them to the glovebox. Bis(cyclopentadienyl)cobalt(II), known as cobaltocene $\left(\mathrm{CoCp}_{2}\right)$, was purchased from Sigma-Aldrich and assayed in dried and degassed $\mathrm{MeCN}$ using a colorimetric method prior to titration experiments. The different oxidation states $-3+, 2+, 1+$ 
and $0-$ of the catenanes $\mathbf{1} \cdot 4 \mathrm{PF}_{6}$ and $\mathbf{2} \cdot 4 \mathrm{PF}_{6}$ were obtained by adding 1.0, 2.0, 3.0 and 4.0 equiv of $\mathrm{CoCp}_{2}$, respectively, in $\mathrm{MeCN}$ solutions: they were characterized simultaneously using UVVis and EPR spectroscopies. In the cases of UV-Vis and EPR experiments, MeCN was used as the solvent, while NMR spectra were recorded in $\mathrm{C}_{6} \mathrm{D}_{5} \mathrm{CD}_{3}$ or $\mathrm{CD}_{3} \mathrm{CN}$. Single crystal $\mathrm{X}$-ray diffraction analysis of the [2]catenanes in their $3+$ to 0 oxidations states $-\mathbf{1}^{0}, \mathbf{2}^{0}, \mathbf{1}^{\bullet / 3+}$ and $\mathbf{2}^{\mathbf{0} 3+}$ — were performed on a Bruker Kappa Apex II CCD diffractometer using $\mathrm{Cu}-\mathrm{K} \alpha(\lambda=0.15418$ $\mathrm{nm}$ ) radiation at $100 \mathrm{~K}$. Structure solutions and refinements were carried out using the Olex 21.2 suite of programs. Data collection and structure refinement details are available in CIF files. $\mathrm{CCDC}$ depositions $\mathrm{X} 1, \mathrm{X} 2, \mathrm{X} 3$ and $\mathrm{X} 4$ contain the supplementary crystallographic data for this manuscript and can be obtained free of charge from the www.ccdc.cam.ac.uk/data_request/cif website.

\section{Solution-State Characterization of [2]Catenanes}

\subsection{UV-Vis Spectroscopy}

A series of solutions with the catenanes in their $3+, 2+, 1+$ and 0 oxidation states were prepared by the chemical reduction of the fully oxidize catenanes using 1.0, 2.0, 3.0 and 4.0 equiv of $\mathrm{CoCp}_{2}$, respectively. The UV-Vis spectra were recorded in solutions with 1:0, 1:1, 1:2, 1:3 and 1:4 molar ratios of the catenane:CoCp $\mathrm{C}_{2}$ in a final volume of $1.00 \mathrm{~mL}$ by using catenane $(100 \mu \mathrm{M})$ and $\mathrm{CoCp}_{2}(1.7 \mathrm{mM})$ solutions in $\mathrm{MeCN}$. UV-Vis Spectra of solutions of $\mathbf{1} \cdot 4 \mathrm{PF}_{6}$ and $\mathbf{2} \cdot 4 \mathrm{PF}_{6}$ at different oxidation states (from $4+$ to 0 ) were recorded in the $300-800 \mathrm{~nm}$ wavelength window using a $2 \mathrm{~mm}$ quartz cuvette.

\subsection{EPR Spectroscopy}

Samples for EPR investigations were prepared as described for the carrying out of the UV-Vis spectroscopy with the exception that the concentration of the stock solution of catenane was maintained at $250 \mu \mathrm{M}$. The EPR spectra of $\mathbf{1} \cdot 4 \mathrm{PF}_{6}$ and $\mathbf{2} \cdot 4 \mathrm{PF}_{6}$ and their reduced states $-3+$ to 0 - were recorded at $298 \mathrm{~K}$ after the chemical reduction with different equiv of $\mathrm{CoCp}_{2}$. 


\subsection{NMR Spectroscopy}

For NMR spectroscopic characterization, samples of the catenanes $\mathbf{1}^{0}$ and $\mathbf{2}^{0}$ were prepared by extraction from a heterogeneous two-phase system. All the experiments were performed in an Ar glovebox as follows. The chloride salts $\mathbf{1 . 4 C l}(12.00 \mathrm{mg}, 7.3 \mu \mathrm{mol})$ and $\mathbf{2} .4 \mathrm{Cl}(10.00 \mathrm{mg}, 8.3$ $\mu \mathrm{mol})$ were dissolved in an aqueous buffer $\left(\mathrm{CO}_{3}{ }^{2-} / \mathrm{HCO}_{3}{ }^{-}\right)$at $\mathrm{pH} 9.0(1.00 \mathrm{~mL})$. The organic phase consisting of $\mathrm{C}_{6} \mathrm{D}_{5} \mathrm{CD}_{3}(1.00 \mathrm{~mL})$ was added to the buffered solution. After adding activated zinc dust as a reducing agent, the bilayer mixture was stirred at room temperature for $30 \mathrm{~min}$. The initial color intensities of the aqueous layers - violet for $\mathbf{1} \cdot 4 \mathrm{Cl}$ and red for $\mathbf{2} \cdot 4 \mathrm{Cl}$, respectively - decreased gradually while the colors of the $\mathrm{C}_{6} \mathrm{D}_{5} \mathrm{CD}_{3}$ layers changed from colorless to light yellow, followed by a dark yellowish-orange color, indicating that the fully reduced catenanes had been extracted into the $\mathrm{C}_{6} \mathrm{D}_{5} \mathrm{CD}_{3}$ layers. After washing with $\mathrm{H}_{2} \mathrm{O}(3 \mathrm{x}$ $1.00 \mathrm{~mL}$ ), the organic layers were transferred in a Wilmad NMR tube and capped. ${ }^{1} \mathrm{H},{ }^{13} \mathrm{C}$, ROESY, DOSY and VT-NMR spectroscopies were performed. See Figure S1 for proton assignments in the ${ }^{1} \mathrm{H}$ NMR spectra.

1 $^{0}:{ }^{1} \mathrm{H}$ NMR (600 MHz, $\left.\mathrm{C}_{6} \mathrm{D}_{5} \mathrm{CD}_{3}, 298 \mathrm{~K}\right) \delta 8.02$ (br, 4H, H $\mathrm{H}_{4 / 8}$ ), 7.20 (br, 4H, $\mathrm{H}_{3 / 7}$ ), 7.10 (s, 8H, $\mathrm{H}_{\mathrm{Xy}}$ ), 6.47 (br, 4H, $\mathrm{H}_{2 / 6}$ ), 5.28 (br, 8H, $\mathrm{H}_{\alpha / \beta}$ ), 3.96 (br, $\mathrm{OCH}_{2 \mathrm{a}}$ ), 3.60-3.55 (m, OCH $\mathrm{OC}_{2 \mathrm{~d}}, \mathrm{~N}-\mathrm{CH}_{2}$ ).

$\mathbf{2}^{0}:{ }^{1} \mathrm{H}$ NMR (600 MHz, $\left.\mathrm{C}_{6} \mathrm{D}_{5} \mathrm{CD}_{3}, 298 \mathrm{~K}\right) \delta 7.22$ (br, 8H, $\mathrm{H}_{\mathrm{Xy}}$ ), 6.57 (s, 8H, H $\mathrm{HQ}_{\mathrm{HQ}}$ ), 5.43(br, 8H, $\left.\mathrm{H}_{\alpha}\right), 5.31\left(\mathrm{br}, 8 \mathrm{H}, \mathrm{H}_{\beta}\right), 3.85\left(\mathrm{t}, J=5.4 \mathrm{~Hz}, 8 \mathrm{H}, \mathrm{OCH}_{2 \mathrm{a}}\right), 3.65\left(\mathrm{br}, 8 \mathrm{H}, \mathrm{N}-\mathrm{CH}_{2}\right), 3.57$ (t, $J=5.4 \mathrm{~Hz}$, $\left.8 \mathrm{H}, \mathrm{OCH}_{2 \mathrm{~b}}\right), 3.53\left(\mathrm{~s}, 16 \mathrm{H}, \mathrm{OCH}_{2 \mathrm{c}, \mathrm{d}}\right)$.

In order to evaluate the molecular dynamics of $\mathbf{2}^{0}$ and compare it with its fully-oxidized counterpart $\mathbf{2}^{4+}$ in solution, VT-NMR spectroscopy (Figure S2) was performed. In the case of both $\mathbf{1}^{0}$ and $\mathbf{1}^{4+}$, peak broadening, coalescence and reappearing with sharpening of the ${ }^{1} \mathrm{H}$ NMR resonances were observed (Figures S2 and S3) with changes in temperature. The VT-NMR spectra of $\mathbf{1}^{0}$ in $\mathrm{C}_{6} \mathrm{D}_{5} \mathrm{CD}_{3}$ show only a one set of peaks for both rings indicating that the DNP38C10 and $\mathrm{CBPQT}^{0}$ rings are undergoing fast rotation on the ${ }^{1} \mathrm{H}$ NMR time-scale. By contrast, the rings in $\mathbf{1}^{4+}$ undergo slow circumrotations on the ${ }^{1} \mathrm{H}$ NMR time-scale. See also ref $\mathrm{S} 2$. 

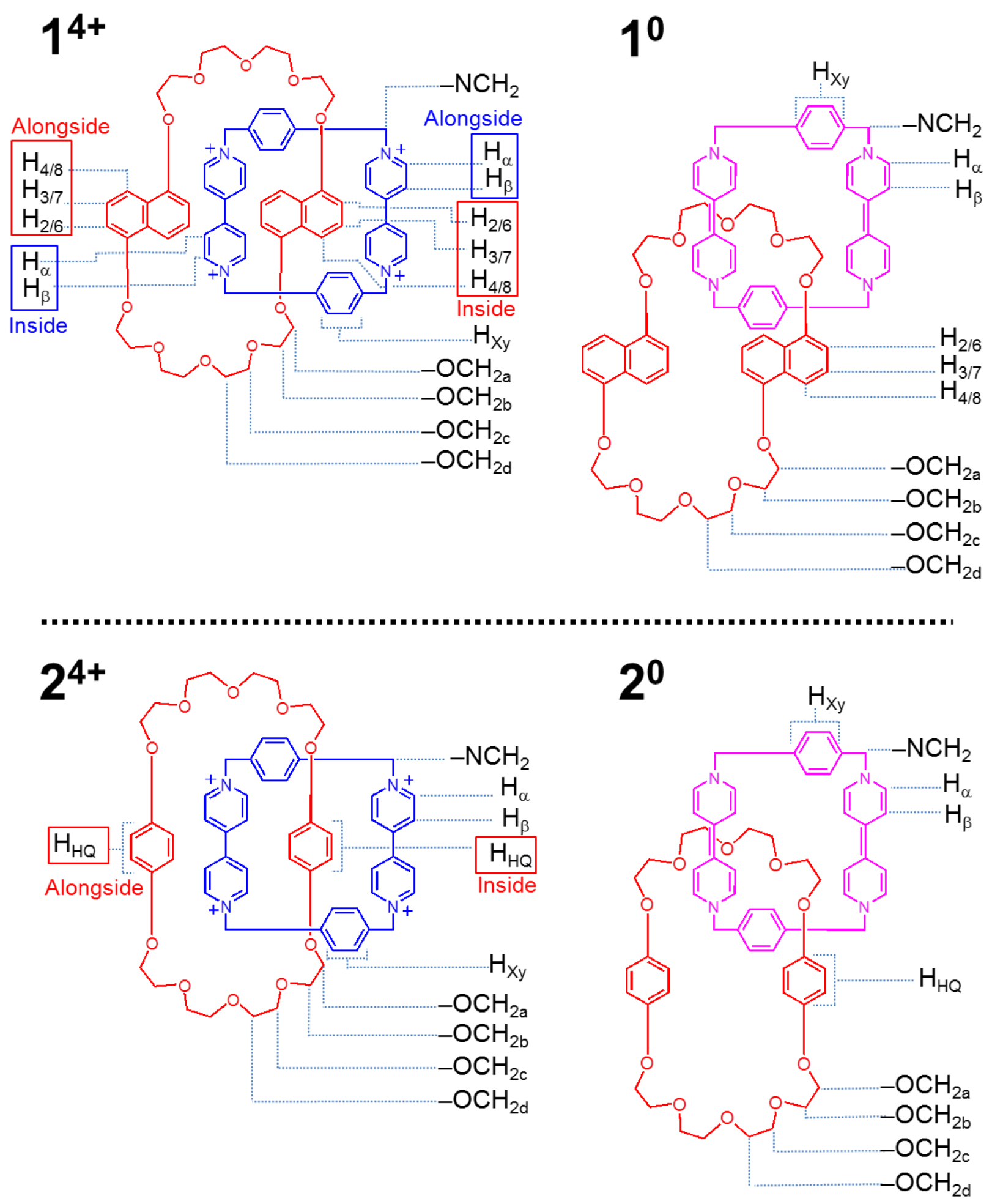

Figure S1. Proton labels on the structural formulas of the [2] catenanes $\mathbf{1}^{4+}, \mathbf{1}^{0}, \mathbf{2}^{4+}$ and $\mathbf{2}^{0}$. 

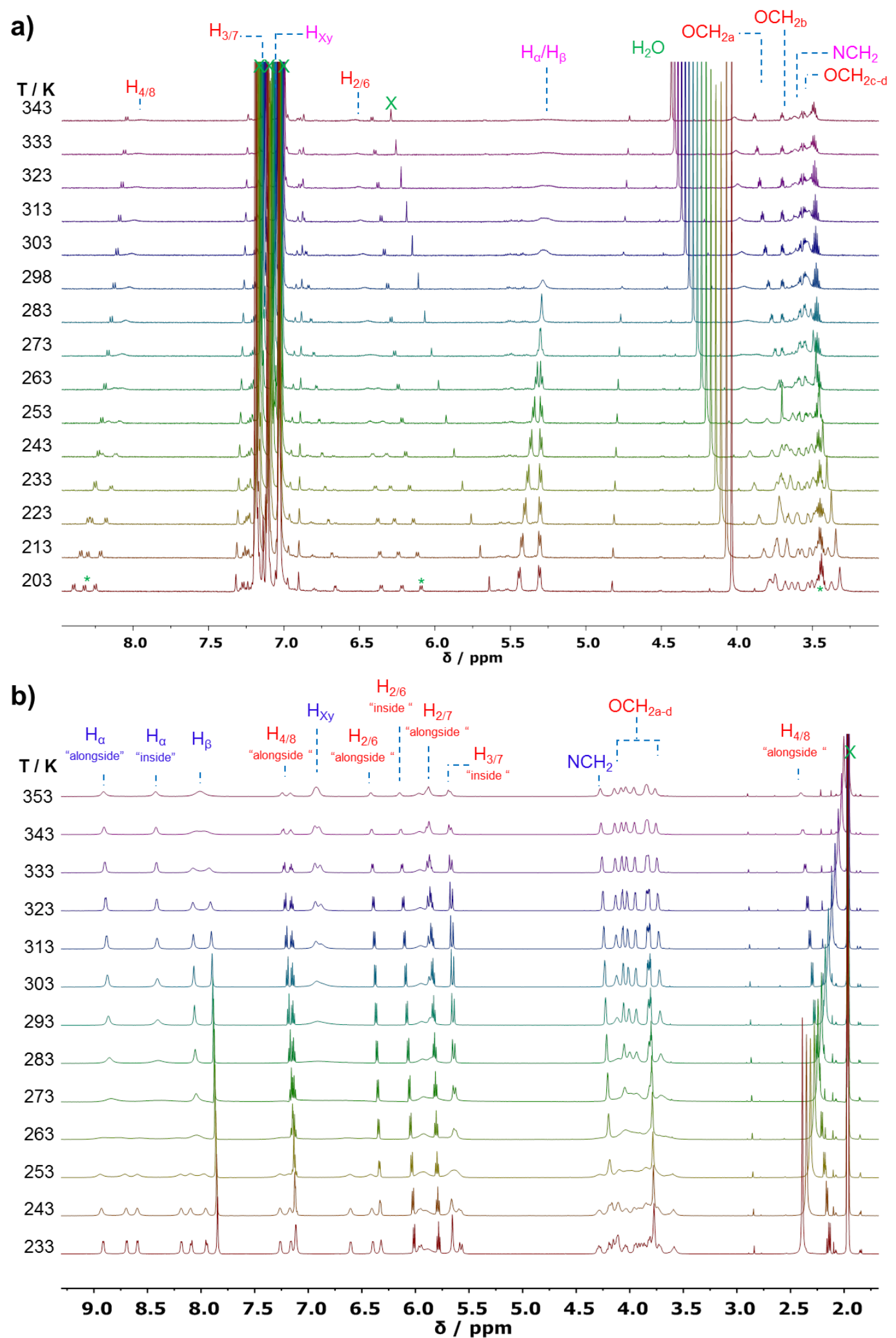

Figure S2. Variable temperature ${ }^{1} \mathrm{H}$ NMR spectra $(600 \mathrm{MHz})$ of (a) $\mathbf{1}^{0}$ recorded in $\mathrm{C}_{6} \mathrm{D}_{5} \mathrm{CD}_{3}$ and (b) $\mathbf{1}^{4+}$ recorded in $\mathrm{CD}_{3} \mathrm{CN}$. 
a)

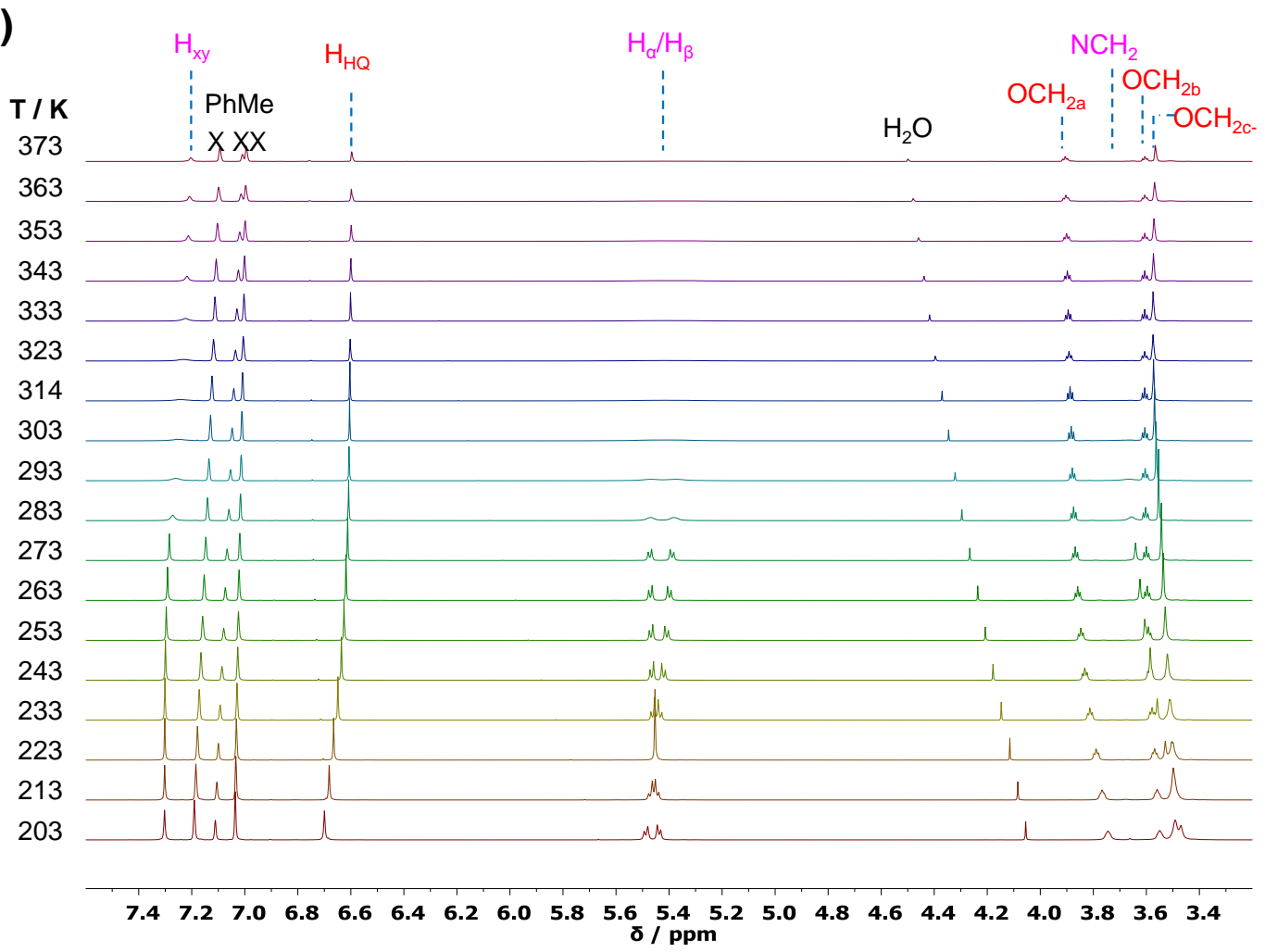

b)

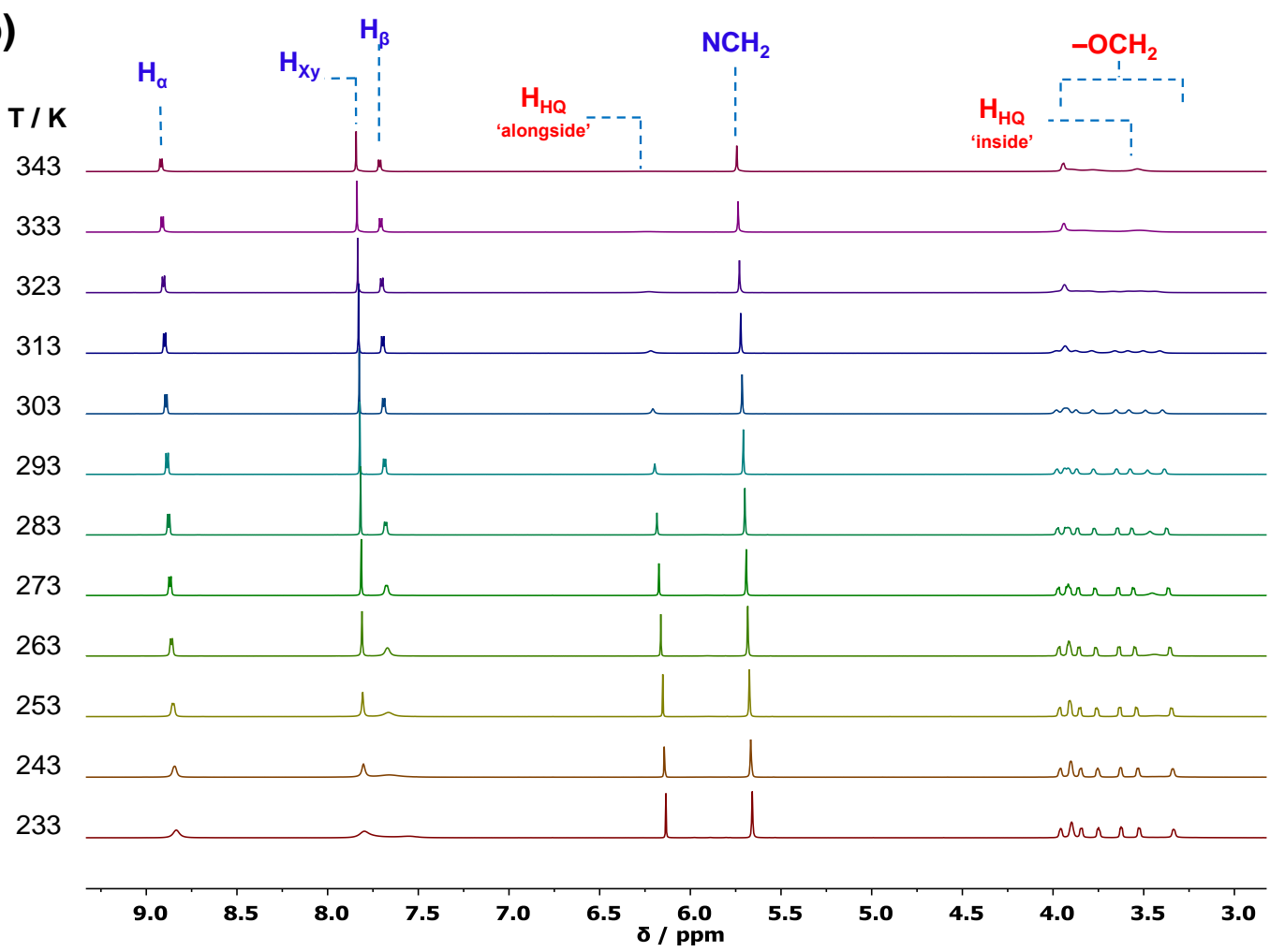

Figure S3. Variable temperature ${ }^{1} \mathrm{H}$ NMR spectra $(600 \mathrm{MHz})$ of (a) $2^{0}$ recorded in $\mathrm{C}_{6} \mathrm{D}_{5} \mathrm{CD}_{3}$ and (b) $\mathbf{2}^{4+}$ recorded in $\mathrm{CD}_{3} \mathrm{CN}$. 
${ }^{1} \mathrm{H}$ DOSY (Figure S4 and S5) and ${ }^{1} \mathrm{H}-{ }^{1} \mathrm{H}$ ROESY (Figure S6 and S7) NMR spectra of $\mathbf{1}^{0}$ and $\mathbf{2}^{0}$ were performed at low temperature $(248 \mathrm{~K})$ to minimize the molecular-level dynamic phenomena.

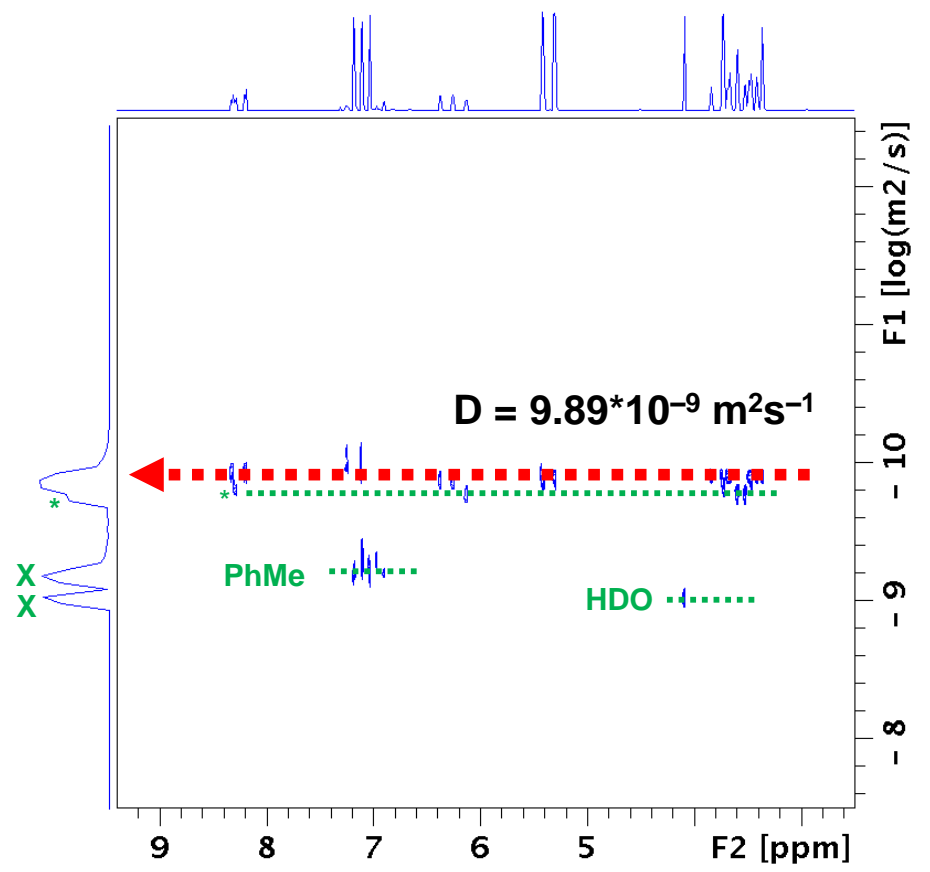

Figure S4. ${ }^{1} \mathrm{H}$ DOSY NMR spectrum of $\left(600 \mathrm{MHz}, \mathrm{C}_{6} \mathrm{D}_{5} \mathrm{CD}_{3}, 248 \mathrm{~K}\right)$ of $\mathbf{1}^{0}$. *The sample contains small amount of DNP38C10 impurities.

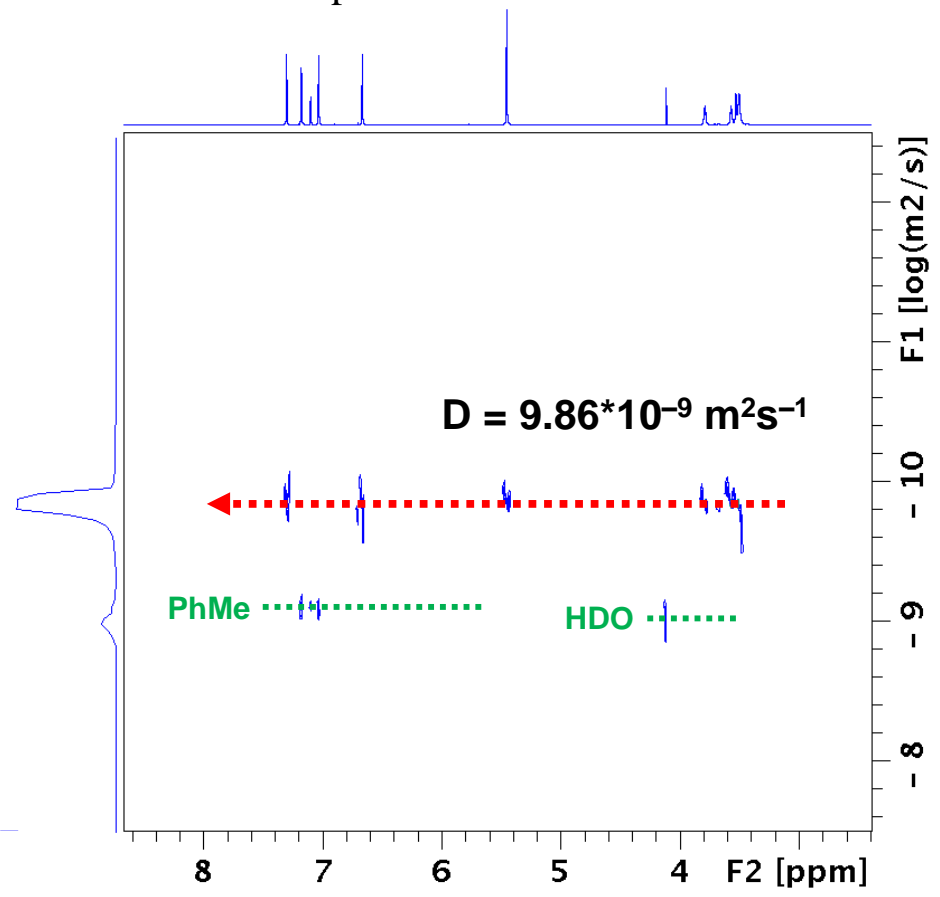

Figure S5. ${ }^{1} \mathrm{H}$ DOSY NMR spectrum of $\left(600 \mathrm{MHz}, \mathrm{C}_{6} \mathrm{D}_{5} \mathrm{CD}_{3}, 248 \mathrm{~K}\right)$ of $\mathbf{2}^{0}$. 


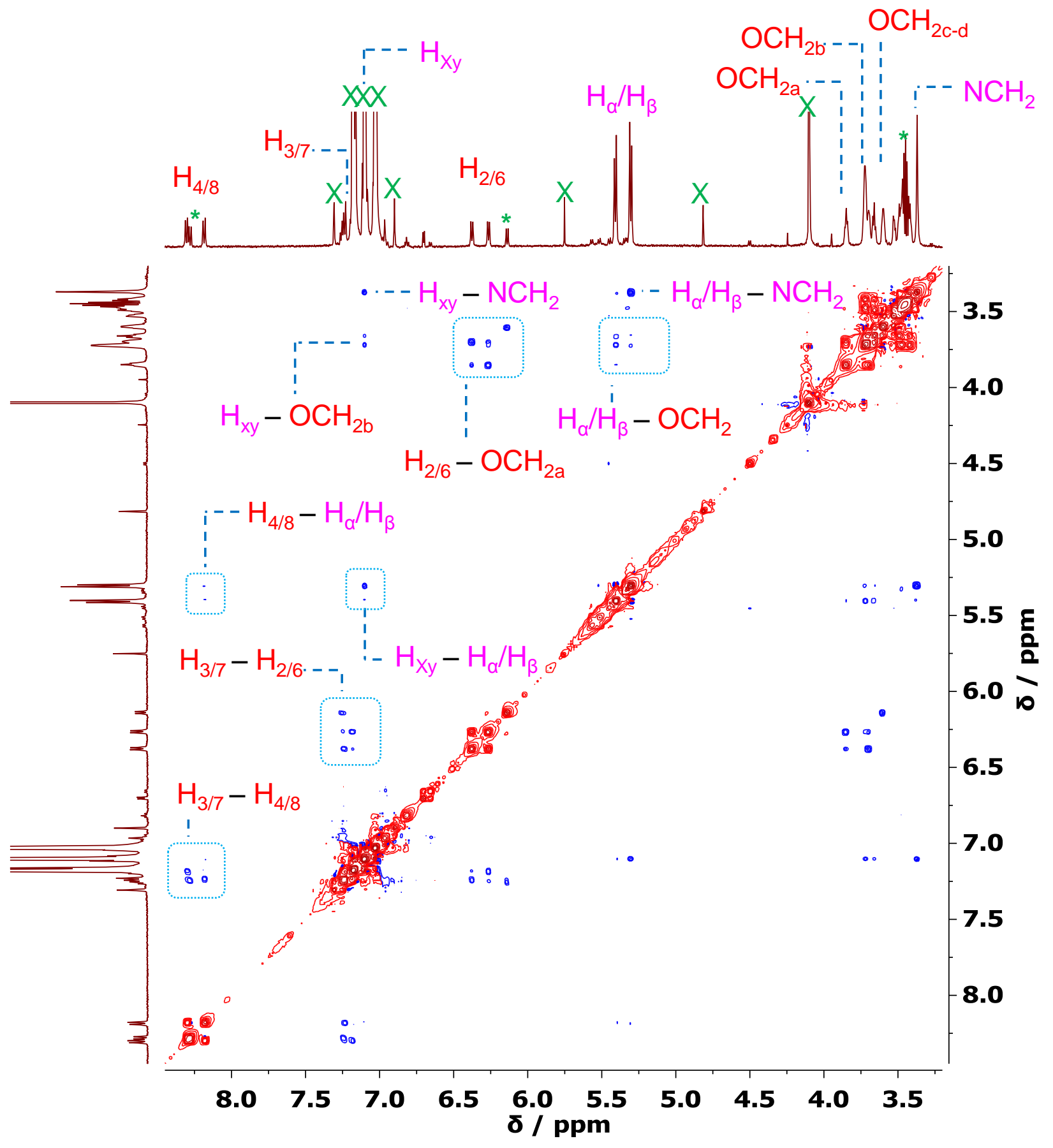

Figure S6. ${ }^{1} \mathrm{H}-{ }^{1} \mathrm{H}$ ROESY spectrum of $\left(600 \mathrm{MHz}, \mathrm{C}_{6} \mathrm{D}_{5} \mathrm{CD}_{3}, 248 \mathrm{~K}\right)$ of $\mathbf{1}^{0}$. 


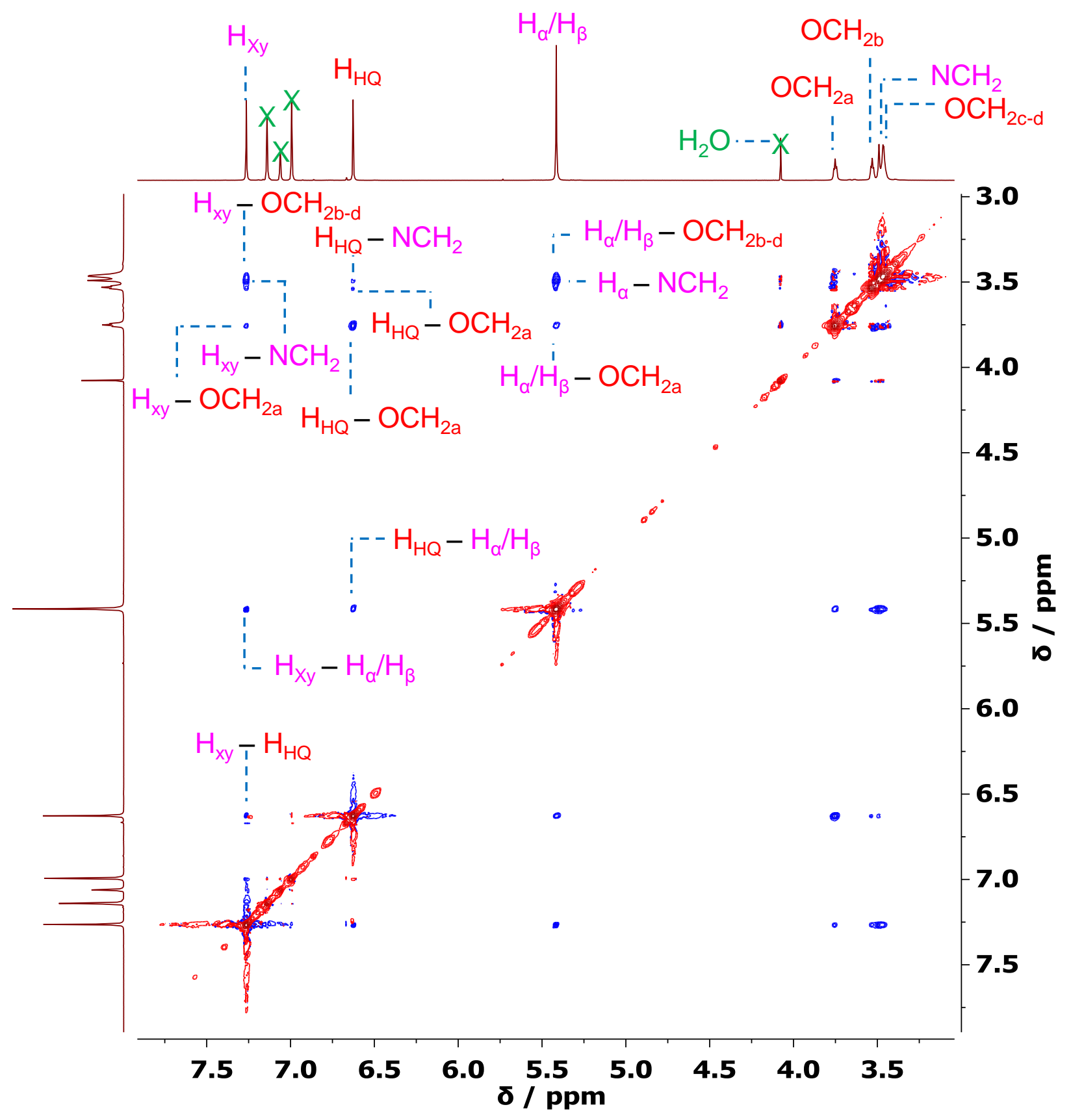

Figure S7. ${ }^{1} \mathrm{H}-{ }^{1} \mathrm{H}$ COSY spectrum of $\left(600 \mathrm{MHz}, \mathrm{C}_{6} \mathrm{D}_{5} \mathrm{CD}_{3}, 248 \mathrm{~K}\right)$ of $\mathbf{2}^{0}$. 


\section{Solid-State Characterization of [2]Catenanes \\ 3.1. Crystal Growth}

Unless otherwise stated, the crystals of [2]catenanes in the different oxidation states were grown in an Ar glovebox at $273 \mathrm{~K}$.

$\mathbf{1}^{0}: \mathbf{1} \cdot 4 \mathrm{PF}_{6}(4.75 \mathrm{mg}, 2.7 \mathrm{mmol})$ were dissolved in $\mathrm{MeCN}(1.00 \mathrm{~mL})$ and 5.0 equiv of saturated $\mathrm{CoCp}_{2}(110 \mu \mathrm{L})$ in $\mathrm{MeCN}$ were added. The solution was filtered. After $1 \mathrm{~h}$ standing at room temperature, dark orange colored elongated cubes of $\mathbf{1}^{0}$ were obtained in quantitative yield. Vapor diffusion of ${ }^{i} \mathrm{Pr}_{2} \mathrm{O}$ into a dilute solution of the fully reduced $\mathbf{1}^{0}$ catenane in $\mathrm{MeCN}$ yielded the same single crystal of $\mathbf{1}^{0}$ as confirmed by the unit cell parameters.

$\mathbf{2}^{0}$ : A solution of $\mathbf{2}^{0}$ in PhMe was obtained using a bilayer extraction method, starting with $\mathbf{2} \cdot 4 \mathrm{Cl}$ (5.00 $\mathrm{mg}, 3.0 \mathrm{mmol}$ ) as explained in Section 2.3 for the sample preparation prior to NMR spectroscopic investigations. Diffusion of $n$-hexane vapor into a solution of $\mathbf{2}^{0}$ in PhMe yielded bright orange-colored elongated cubic-shaped single crystals of $\mathbf{2}^{0}$ in quantitative yield.

1.3PF $: 1 \cdot 4 \mathrm{PF}_{6}(3.30 \mathrm{mg}, 1.9 \mathrm{mmol})$ were dissolved in $\mathrm{MeCN}(660 \mu \mathrm{L})$ and reduced with activated $\mathrm{Zn}$ dust. After $30 \mathrm{~min}$ of stirring at room temperature, the solution was filtered and subjected to vapor diffusion with ${ }^{i} \operatorname{Pr}_{2} \mathrm{O}$. Within 2 weeks, dark violet-purple colored needles of $1 \cdot 3 \mathrm{PF}_{6}$ were obtained in quantitative yield.

2•3PF $: 2 \cdot 4 \mathrm{PF}_{6}(3.50 \mathrm{mg}, 2.1 \mathrm{mmol})$ were dissolved in $\mathrm{MeCN}(660 \mu \mathrm{L})$ and reduced with activated $\mathrm{Zn}$ dust. After $30 \mathrm{~min}$ of stirring at room temperature, the solution was filtered and subjected to vapor diffusion with ${ }^{i} \operatorname{Pr}_{2} \mathrm{O}$. Within 2 weeks, dark violet-purple colored needles of $2 \cdot 3 \mathrm{PF}_{6}$ were obtained in quantitative yield. 


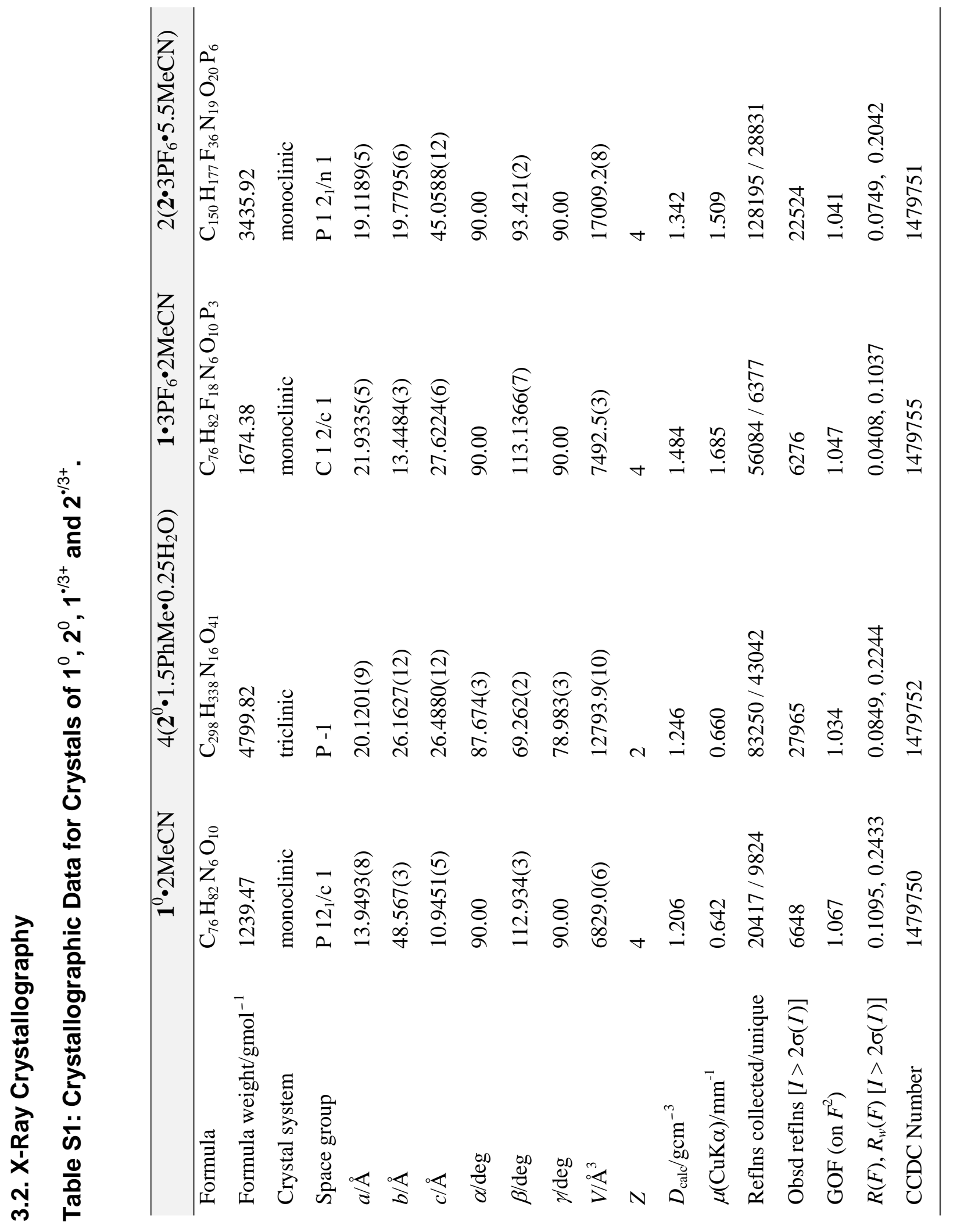


The extended solid-state superstructure consisting of the cell unit of $\mathbf{2}^{\mathbf{0}}$ is shown in Figure S8.

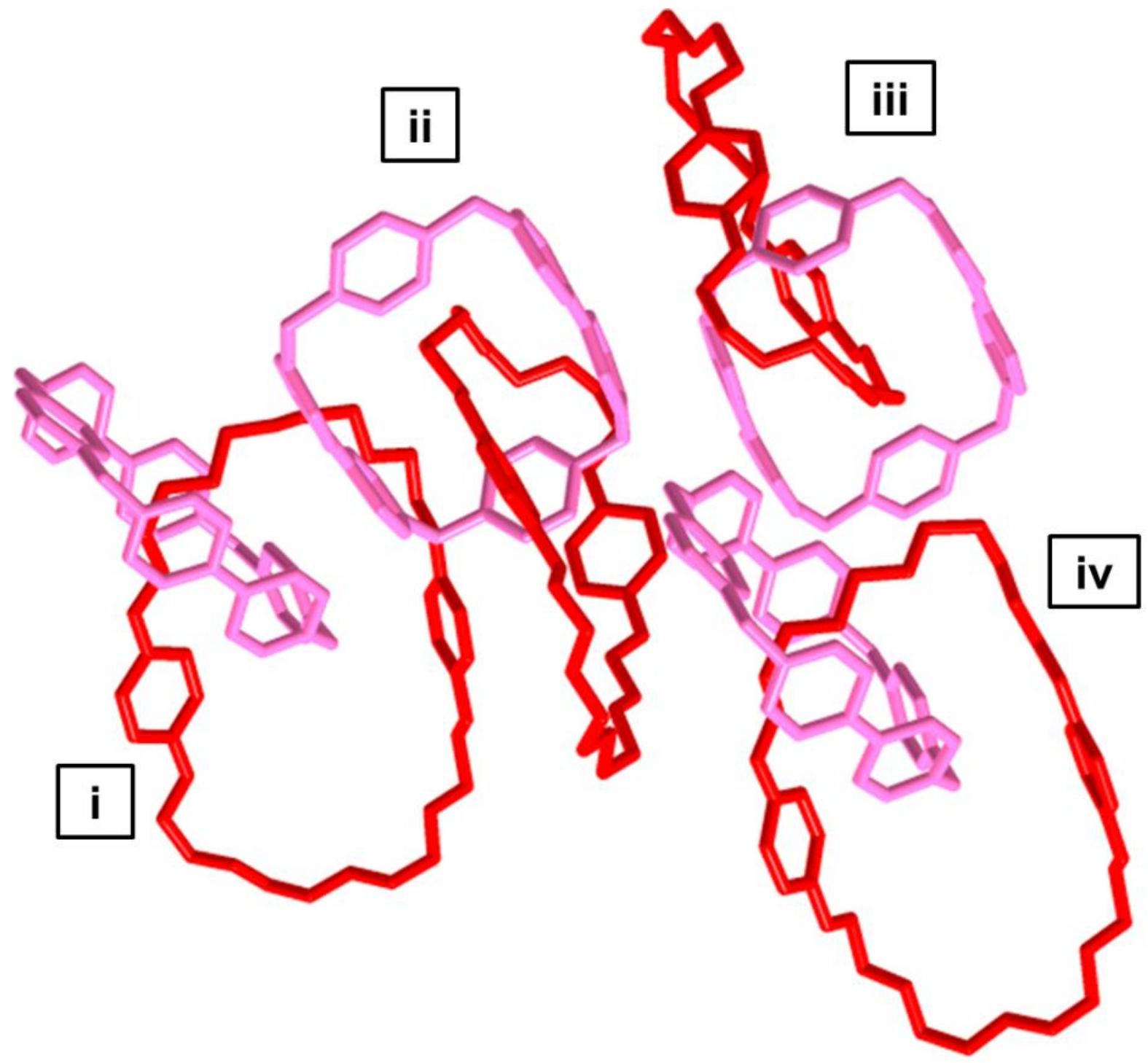

Figure S8. Plan view of the superstructure presents in the unit cell of the $\mathbf{2}^{\mathbf{0}}$ catenane in a tubular representation displaying the four different co-conformations of the catenane.

Four different co-conformations of the neutral catenane $\mathbf{2}^{\mathbf{0}}$ are present in the unit cell. In two coconformations (Figure S9, ii and iii) the BIPY unit of the $\mathrm{CBPQT}^{0}$ sits around the first $\mathrm{CH}_{2}-\mathrm{O}-$ $\mathrm{CH}_{2}$ linker of the glycol chain, while in the other two co-conformations (Figure S9, i and iv) the ring sits on the second $\mathrm{CH}_{2}-\mathrm{O}-\mathrm{CH}_{2}$ linker of the glycol chain. 

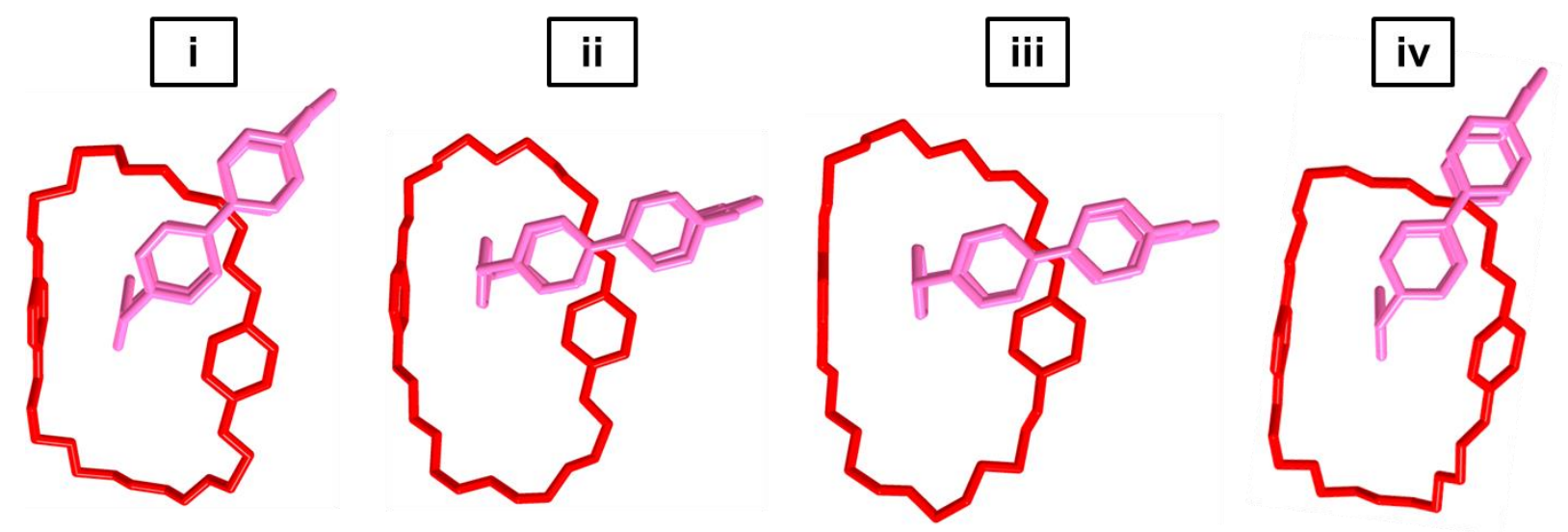

Figure S9. Side-on views of the tubular representation of the four co-conformations present in the unit cell of the $\mathbf{2}^{\mathbf{0}}$ catenane.

\section{Computational Analysis}

In order to examine the stability of catenanes resulting from chemical reduction, quantummechanical modeling was performed. Density functional theory (DFT) calculations at the level of M06-HF/6-311++G**//M06-HF/6-31G* were employed in order to obtain the predicted geometries of the $4+$ to 0 redox states of the catenanes $\mathbf{1}^{4+}$ and $\mathbf{2}^{4+}$ with Poison-Boltzmann solvation model.

The TD-DFT calculations were carried out on the truncated models of $\mathbf{1}^{4+}$ and $\mathbf{2}^{4+}$, obtained by removing the glycol chain following re-optimization of the structure at the level of BLYP+LC $(\mu=0.2)^{\mathrm{S} 6} / 6-31 \mathrm{G}(\mathrm{d})$, which was found to predict accurate excitation energy of the complex between the CBPQT and tetrathiafulvalene (TTF). ${ }^{\mathrm{S} 7}$ The strongest absorption below $450 \mathrm{~nm}$ for both complexes corresponds to the HOMO (located on the electron-rich guests) to LUMO and LUMO+1 (located on the electron-poor $\mathrm{CBPQT}^{4+}$ ring), as illustrated in Figure S10. The excitation energies for $\mathbf{1}^{4+}$ and $\mathbf{2}^{4+}$ are 557 and $530 \mathrm{~nm}$.

The energy decomposition analysis was carried out with Q-Chem 4.1.0.1 ${ }^{\mathrm{S} 8}$ at M06-HF/6$311 \mathrm{G} * * / / \mathrm{M} 06-\mathrm{HF} / 6-31 \mathrm{G}^{*}$ level. 

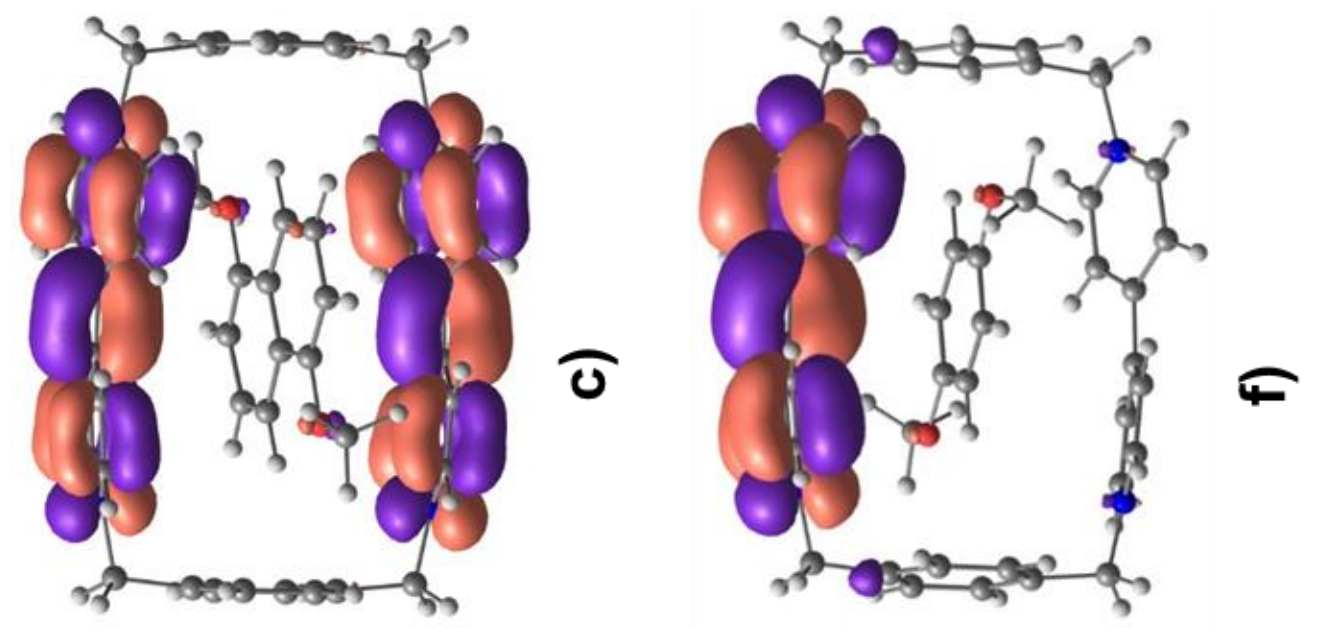

4
0
0
0
3
0
0
0
0
0
0
0
0
0
0
0
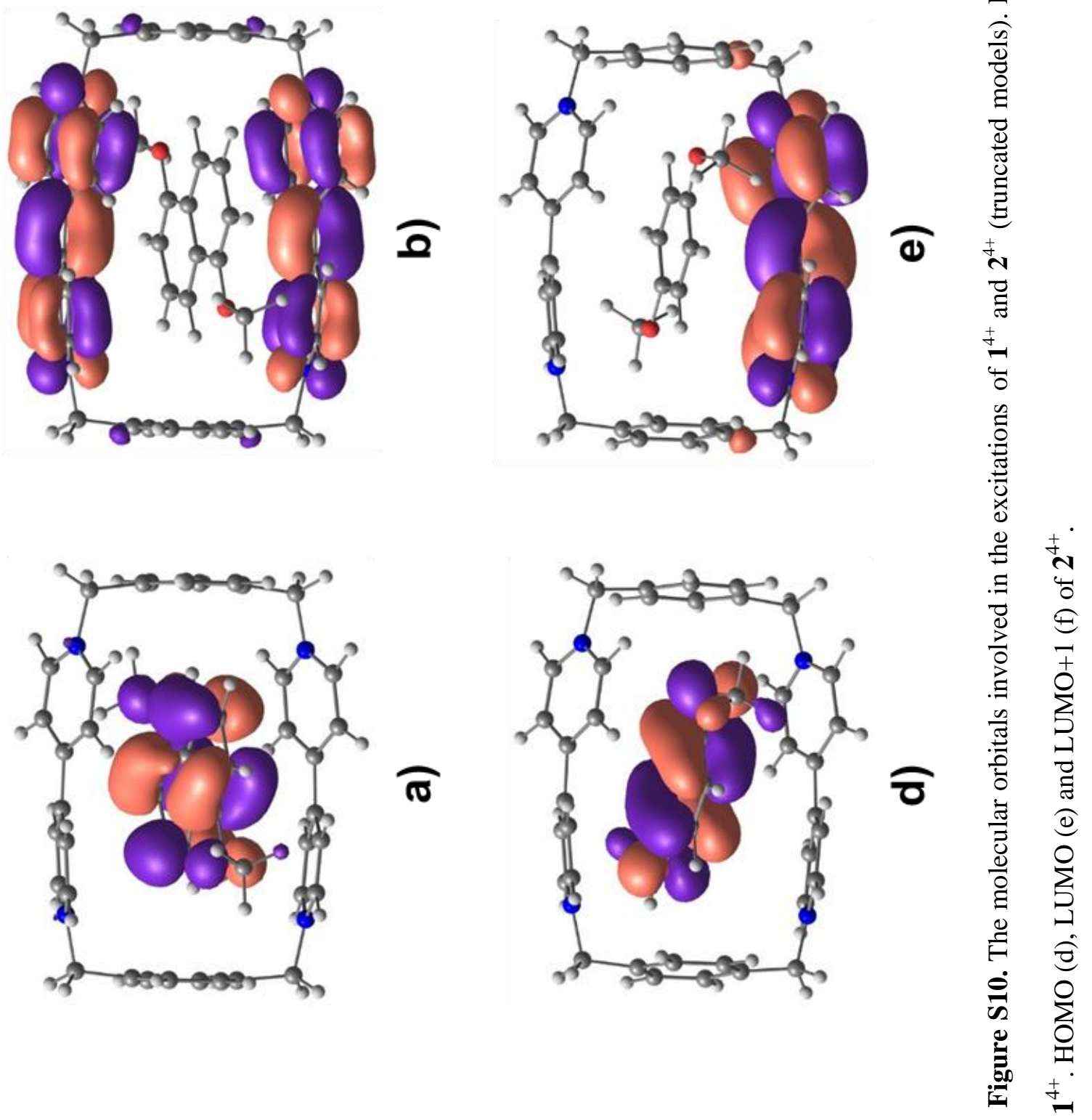
The energy optimized geometries for the calculated structures $\mathbf{1}^{\mathbf{0} 3+}$ and $\mathbf{2}^{\mathbf{6} 3+}$ are shown in Figure S11 and Figure S12, respectively.
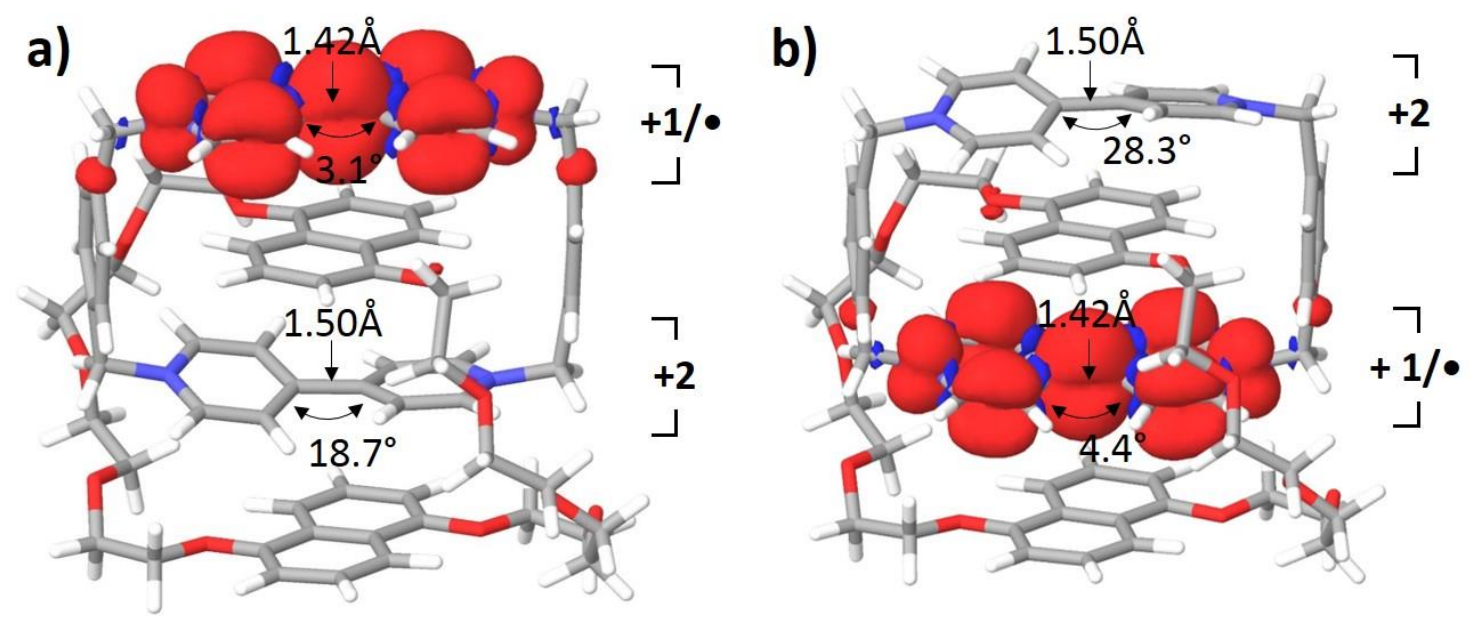

Figure S11. Calculated spin density for two co-conformations of $\mathbf{1}^{\mathbf{0} 3+}$. (a) The lower-energy coconformation with the radical cationic $\mathrm{BIPY}^{*+}$ alongside and the dicationic $\mathrm{BIPY}^{2+}$ inside the DNP38C10 ring. (b) The higher-energy co-conformation $\left(5 \mathrm{kcal} \mathrm{mol}^{-1}\right)$ with the radical cationic BIPY ${ }^{*}$ inside and the dicationic $\mathrm{BIPY}^{2+}$ outside the DNP38C10 ring. The bond lengths and the torsional angles around the $\mathrm{C} 4-\mathrm{C} 4$ ' bond of the BIPY units are shown.

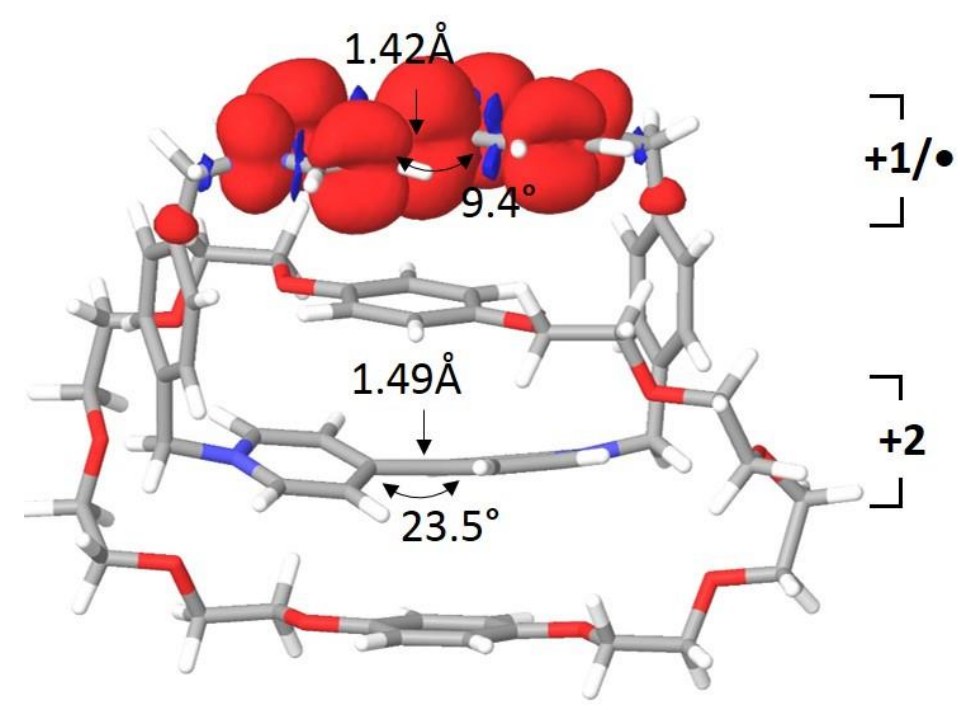

Figure S12. Calculated spin density for the co-conformations of $2^{\circ / 3+}$ with the radical cationic BIPY ${ }^{\circ+}$ alongside and the dicationic BIPY ${ }^{2+}$ inside the macrocyclic polyether. The reverse co-conformation with the radical cationic BIPY ${ }^{\circ+}$ inside and the dicationic $\mathrm{BIPY}^{2+}$ outside the macrocycle is unstable regardless the initial geometries choose and it converges to the former conformation. The bond lengths and the torsional angles around the $\mathrm{C} 4-\mathrm{C} 4$ ' bond of the BIPY units are shown. 
The calculated structures for two co-conformation of the catenanes $\mathbf{1}^{\mathbf{2 ( + )}}$ (a, b) $\mathbf{2}^{\mathbf{2 ( \bullet + )}}$ (Figure S13) show that the diradical dicationic $\mathrm{CBPQT}^{2(\cdot+)}$ rings encircle the aromatic units of crown ethers.
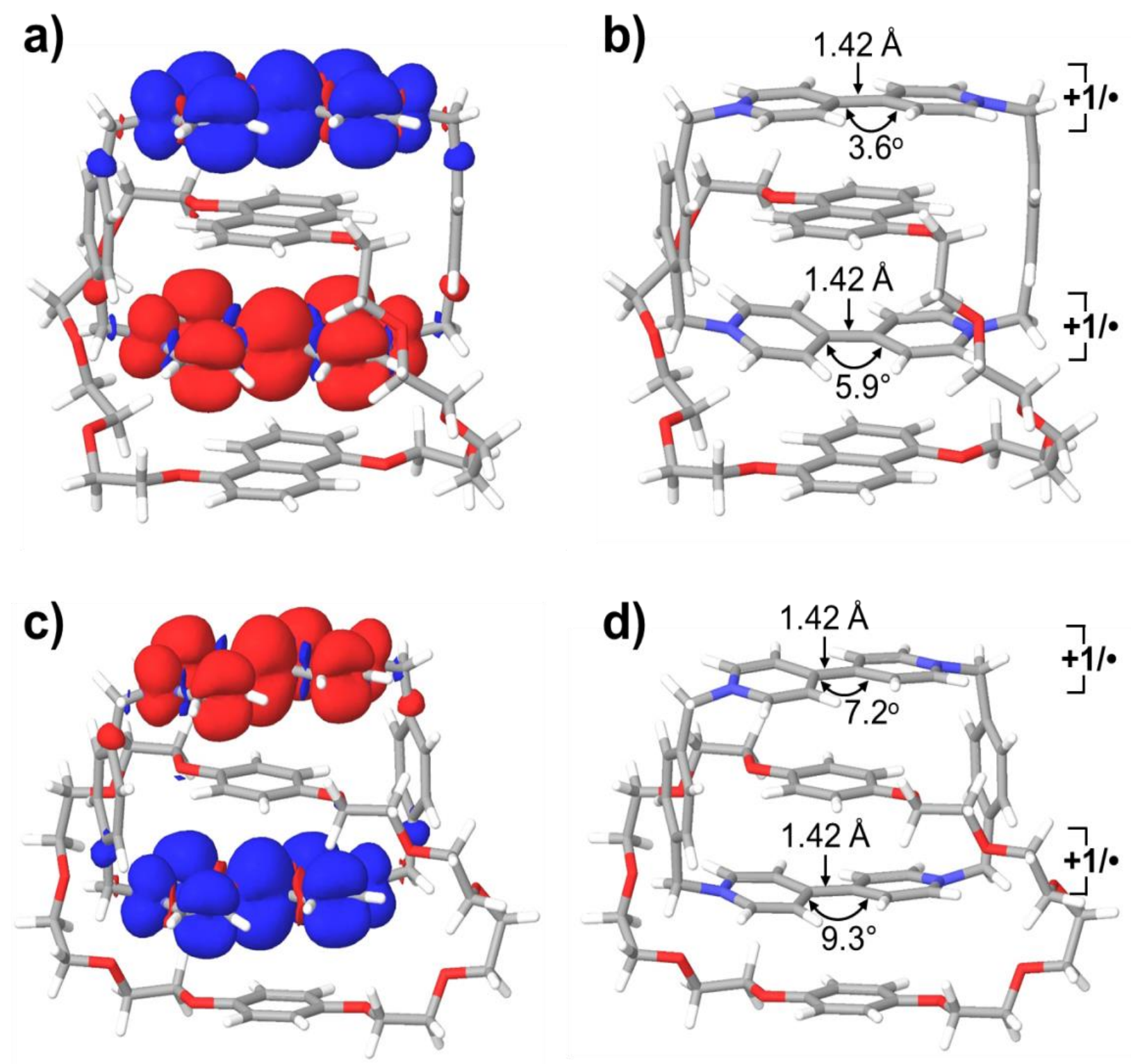

Figure S13. Calculated spin density for two co-conformations of $\mathbf{1}^{\mathbf{2 ( + )}}(\mathrm{a}, \mathrm{b}) \mathbf{2}^{\mathbf{2 ( + )}}(\mathrm{c}, \mathrm{d})$. Spin population of $\mathbf{1}^{\mathbf{2 ( + )}}$ (a) and $\mathbf{2}^{2(+)}(\mathrm{c})$. Calculated geometries of $\mathbf{1}^{\mathbf{2 ( + )}}(\mathrm{b})$ and $\mathbf{2}^{\mathbf{2 ( + )}}(\mathrm{d})$ showing the bond lengths and the torsional angles around the $\mathrm{C} 4-\mathrm{C} 4$ ' bond of the BIPY units. 
The energy optimized geometries for the calculated structures of $\mathbf{1}^{\mathbf{*}}$ and $\mathbf{2}^{\mathbf{*}}$ are shown in figure $\mathrm{S} 14$ and S15, with bond distances and torsional angles around the $\mathrm{C} 4-\mathrm{C} 4$ ' bond of the BIPY units.
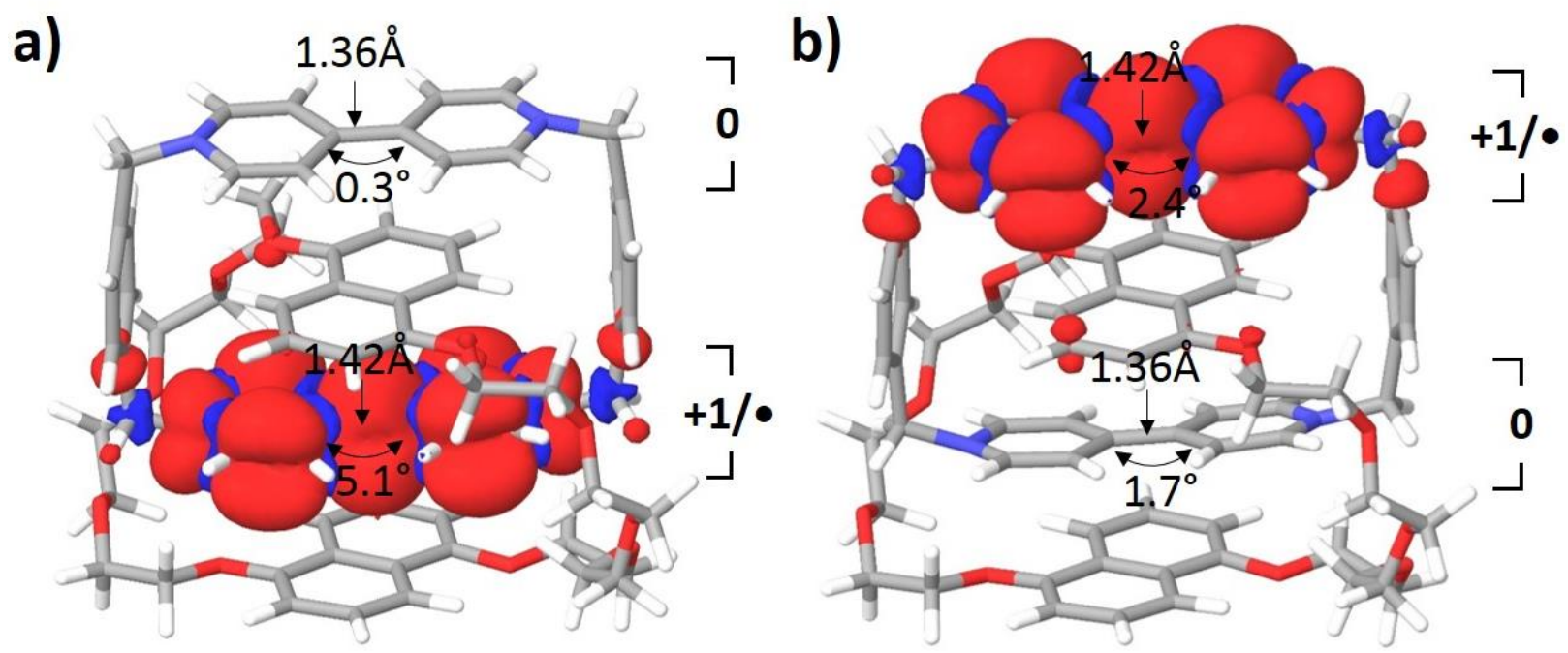

Figure S14. Calculated spin density for two co-conformations of $\mathbf{1}^{\mathbf{*}}$. (a) The lower-energy coconformation with the radical cationic BIPY ${ }^{\circ+}$ located inside the DNP38C10 ring. (b) The higher-energy co-conformation $\left(9.3 \mathrm{kcal} \mathrm{mol}^{-1}\right)$ with the radical cationic BIPY ${ }^{\circ+}$ located outside the DNP38C10 ring. The bond lengths and the torsional angles around the $\mathrm{C} 4-\mathrm{C} 4$ ' bond of the BIPY units are shown.

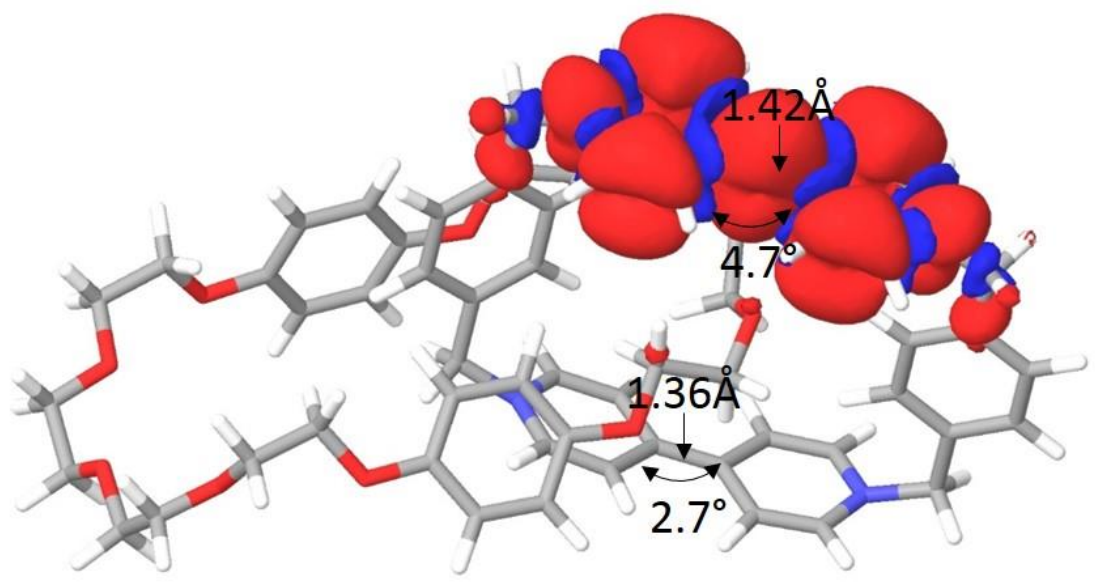

Figure S15. Calculated spin density of $\mathbf{2}^{\cdot+}$ with the radical cationic $\mathrm{BIPY}^{{ }^{+}}$alongside and the dicationic $\mathrm{BIPY}^{2+}$ inside the macrocyclic polyether. 
In order to investigate the stability of the neutral catenanes $\mathbf{1}^{\mathbf{0}}$ and $\mathbf{2}^{\mathbf{0}}$ in different coconformations, the potential energy surface for the sliding of the CBPQT ring through the crown ether was compared (Figure S16) to the energy for the movement of the $\mathrm{CBPQT}^{4+}$ ring in the fully oxidized catenane.

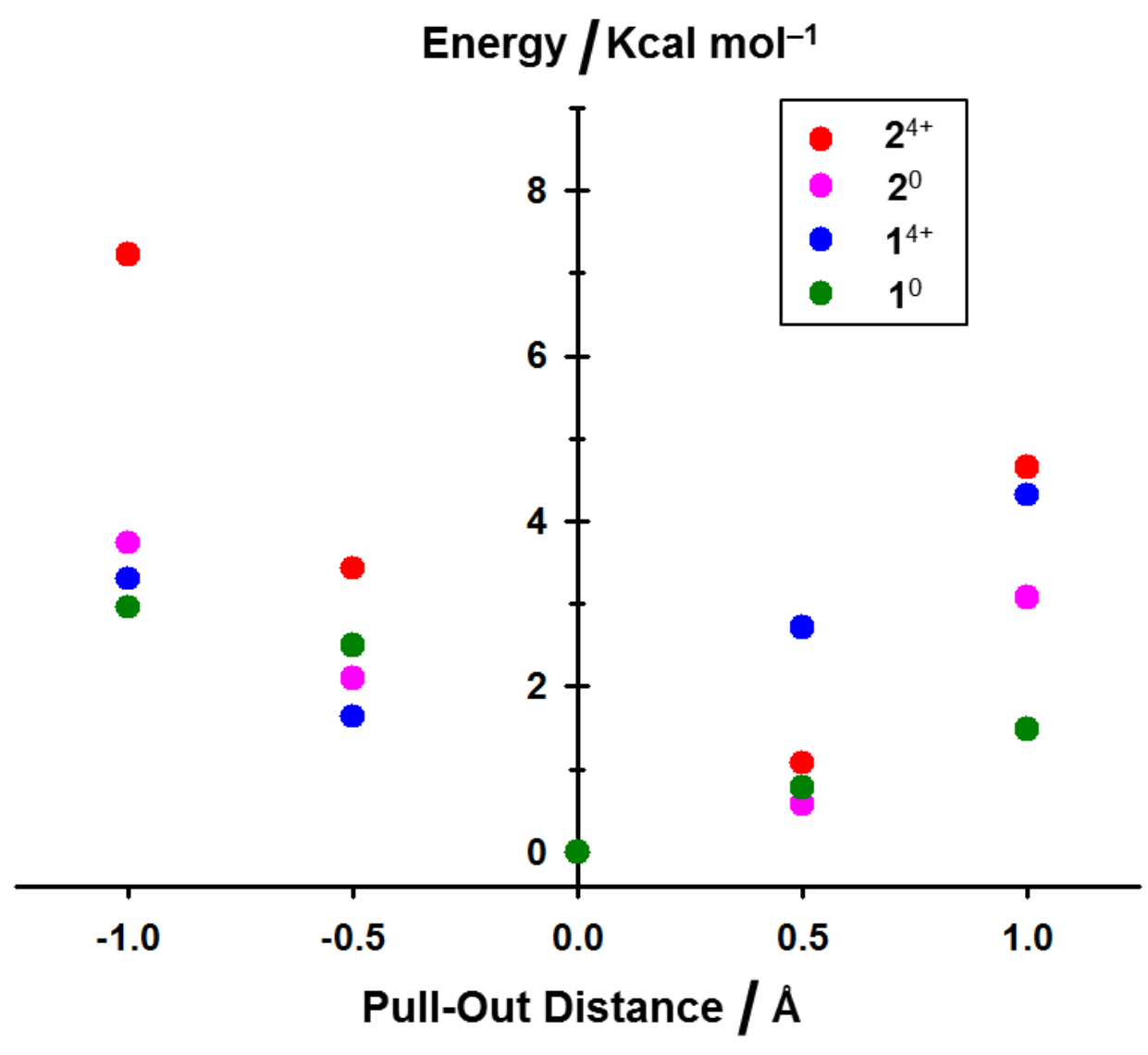

Figure S16. Potential energy surface calculated for the sliding of the CBPQT ring through the crown ether for the neutral and tetracationic catenanes $\mathbf{1}^{4+}$ and $\mathbf{2}^{4+}$. The potential energy surfaces of the neutral and tetracationic catenanes at different conformations were calculated by pushing away the CBPQT ring from the equilibrium position by 0.5 and $1.0 \AA$ in the direction perpendicular to the plane defined by the carbons in four ethylene units and then optimized with all other coordinates relaxed.

The coordinates of optimized complexes were reported in XYZ format. The energies were reported in the following format: (1) M06-HF/6-31G* (in solution), (2) M06-HF/6-31G* (in gas phase), (3) M06-HF/6-311++G** (in gas phase), and (4) the total energy, which is (1)-(2)+(3). 


$$
\begin{array}{llll}
\mathbf{1}^{4+} \text { CBPQT circling DNP } & & \\
-3759.127058 & -3758.479648 & -3759.497667 & -3760.145077 \\
\text { O1 } & 8.4307055988000 & 13.9187421606000 & 5.0742991740000 \\
\text { C2 } & 9.8227822705000 & 14.1547583580000 & 4.8957210800000 \\
\text { C3 } & 7.9569284090000 & 13.9810048832000 & 6.3459677018000 \\
\text { C4 } & 10.2049349068000 & 13.9637093546000 & 3.4387820486000 \\
\text { C5 } & 6.5342234168000 & 13.8147398869000 & 6.4831825336000 \\
\text { C6 } & 8.7374838604000 & 14.2060259501000 & 7.4545073909000 \\
\text { O7 } & 9.5930945679000 & 14.9470852556000 & 2.6163921975000 \\
\text { C8 } & 5.7085942937000 & 13.5712254046000 & 5.3501355055000 \\
\text { C9 } & 5.9612611933000 & 13.9261755626000 & 7.7667855226000 \\
\text { C10 } & 8.1336613049000 & 14.2915023073000 & 8.7391244330000 \\
\text { C11 } & 10.3581194201000 & 15.2818831677000 & 1.4611097174000 \\
\text { C12 } & 4.3530829659000 & 13.4877192402000 & 5.5123591480000 \\
\text { C13 } & 4.5364683983000 & 13.7854161834000 & 7.9022481470000 \\
\text { C14 } & 6.7814115693000 & 14.1699303557000 & 8.9042912532000 \\
\text { C15 } & 9.4095001341000 & 15.5667448321000 & 0.3006979645000 \\
\text { C16 } & 3.7474058862000 & 13.6026617501000 & 6.7923845596000 \\
\text { O17 } & 4.0836752128000 & 13.8384965996000 & 9.1788484926000 \\
\text { O18 } & 8.4236873921000 & 16.5355794600000 & 0.6157421176000 \\
\text { C19 } & 2.6869297100000 & 13.7091091812000 & 9.4137344930000 \\
\text { C20 } & 8.8933628292000 & 17.8713258854000 & 0.7298809311000 \\
\text { C21 } & 1.9435342632000 & 15.0212603529000 & 9.1824924866000 \\
\text { C22 } & 7.7525421052000 & 18.8137796213000 & 0.3838749862000 \\
\text { O23 } & 2.2608762779000 & 15.8825314759000 & 10.2568688169000 \\
\text { C25 } & 1.6123470254000 & 17.1368833449000 & 10.1872041633000
\end{array}
$$



C26 $5.7250235611000 \quad 19.6618348103000 \quad 1.2043000588000$
$\begin{array}{lllll}\text { C27 } & 1.7192571399000 & 17.7928448888000 & 11.5528282804000\end{array}$
$\begin{array}{llll}\text { C28 } & 4.7129340068000 & 19.5241169299000 & 2.3303873990000\end{array}$
O29 $3.0926062802000 \quad 17.889021414700011 .8948046997000$
O30 $\quad 5.4420277270000 \quad 19.6082814597000 \quad 3.5448044072000$
C31 $3.3325387187000 \quad 18.591078020200013 .1068751145000$

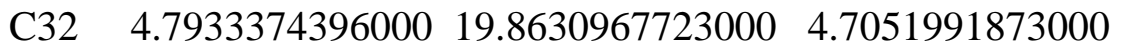
$\begin{array}{lllll}\text { C33 } & 3.6059912899000 & 20.0638568403000 & 12.8271142233000\end{array}$
C34 $5.6655975602000 \quad 20.1573684488000 \quad 5.8068639920000$
C35 $3.4330035685000 \quad 19.8327108597000 \quad 4.8734281900000$
O36 $4.7507247703000 \quad 20.1154739277000 \quad 11.9964400108000$
$\begin{array}{lllll}\text { C37 } & 7.0785145415000 & 20.2106165286000 & 5.6273860785000\end{array}$
$\begin{array}{lllll}\text { C38 } & 5.0962787830000 & 20.3938836757000 & 7.0716858039000\end{array}$
$\begin{array}{llll}\text { C39 } & 2.8823415252000 & 20.0843962289000 & 6.1624369364000\end{array}$
C40 $5.1485225069000 \quad 21.4241594618000 \quad 11.6368239846000$
C41 $7.8810154741000 \quad 20.5093989916000 \quad 6.6930092348000$
C42 $5.9764300844000 \quad 20.6685017216000 \quad 8.1734919085000$
$\begin{array}{lllll}\text { C43 } & 3.6809720324000 & 20.3544151764000 & 7.2402091748000\end{array}$
$\begin{array}{llll}\text { C44 } & 6.1151220227000 & 21.2997362569000 & 10.4725686192000\end{array}$
C45 $\quad 7.3344608387000 \quad 20.7416826279000 \quad 7.9857634764000$
$\begin{array}{llll}\text { O46 } & 5.3550470208000 & 20.8344098398000 & 9.3653895751000\end{array}$
H47 $10.3995169526000 \quad 13.4414533860000 \quad 5.4886023342000$
H48 $10.0690113867000 \quad 15.1716293908000 \quad 5.2104942982000$
H49 $11.2930025348000 \quad 14.0540474774000 \quad 3.3897624255000$
H50 $9.919176650200012 .9645174058000 \quad 3.1001229115000$
H51 $9.8076047933000 \quad 14.3342516954000 \quad 7.3736214243000$
H52 $6.1644844876000 \quad 13.4756424258000 \quad 4.3757176400000$ 

H53 $8.7724938642000 \quad 14.4703160152000 \quad 9.5953162316000$
H54 $10.9915153283000 \quad 16.1431442767000 \quad 1.6851416808000$
H55 $10.9971607028000 \quad 14.4465928237000 \quad 1.1662382702000$
H56 $3.7127761894000 \quad 13.3318024518000 \quad 4.6531495887000$
H57 $8.8681483369000 \quad 14.6559915365000 \quad 0.0476061576000$
H58 $9.9919419933000 \quad 15.8852751611000 \quad-0.5692199676000$
$\begin{array}{lllll}\text { H59 } & 2.5850315954000 & 13.4429048711000 & 10.4629095744000\end{array}$

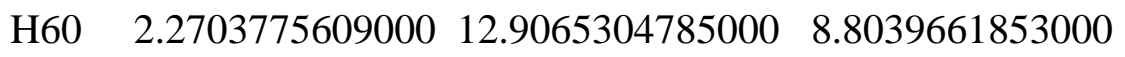
H61 $9.7145628113000 \quad 18.0450655025000 \quad 0.0287884233000$
H62 $9.2425322931000 \quad 18.0710497485000 \quad 1.7462736292000$
H63 2.2396600123000 $15.4704739637000 \quad 8.2289597762000$
H64 $\quad 0.8679443843000 \quad 14.8215789937000 \quad 9.1561366185000$
H65 $8.1313962543000 \quad 19.8401514677000 \quad 0.4091216551000$
H66 $7.3871482175000 \quad 18.5939944510000 \quad-0.6237067124000$
H67 $2.0802186672000 \quad 17.7704104548000 \quad 9.4248705748000$
H68 $\quad 0.5542220624000 \quad 17.0164672584000 \quad 9.9370432174000$

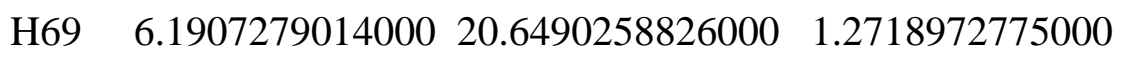
$\begin{array}{lllll}\text { H70 } & 5.2043614436000 & 19.5798353912000 & 0.2453326913000\end{array}$
H71 $1.2542042078000 \quad 18.7809627281000 \quad 11.5124675264000$
H72 $1.1984248921000 \quad 17.1804626704000 \quad 12.2925611857000$
H73 $3.9917417594000 \quad 20.3410042262000 \quad 2.2577250772000$
H74 $4.1839424590000 \quad 18.5708918011000 \quad 2.2718220169000$
H75 2.4848423677000 $18.4856056917000 \quad 13.7877307055000$
H76 $4.2109137111000 \quad 18.144208245600013 .5739768679000$
H77 $3.783588325800020 .5900554359000 \quad 13.7702194355000$
H78 $2.754237709900020 .5262610377000 \quad 12.3197026789000$
H79 $2.7729035398000 \quad 19.6077238357000 \quad 4.0462246366000$ 


\begin{tabular}{llll} 
H80 & 7.4861412387000 & 20.0278653655000 & 4.6425574080000 \\
H81 & 1.8059582689000 & 20.0597199870000 & 6.2776734899000 \\
H82 & 4.2846446730000 & 22.0156451804000 & 11.3221226877000 \\
H83 & 5.6410661134000 & 21.9248988576000 & 12.4749447029000 \\
H84 & 8.9542178982000 & 20.5752002578000 & 6.5666603382000 \\
H85 & 6.9016179307000 & 20.5828398830000 & 10.7230299943000 \\
H86 & 6.5639630993000 & 22.2676581529000 & 10.2407646865000 \\
H87 & 6.3178113701000 & 14.2553349426000 & 9.8792776635000 \\
H88 & 2.6709190658000 & 13.5306078253000 & 6.8644373081000 \\
H89 & 3.2621767520000 & 20.5443462864000 & 8.2187164831000 \\
H90 & 7.9994860976000 & 20.9759227428000 & 8.8057977654000 \\
C91 & 5.7181300777000 & 10.2741602342000 & 6.9988872154000 \\
C92 & 6.8139140792000 & 9.9114799869000 & 6.2076810393000 \\
C93 & 4.4746415003000 & 10.4005826258000 & 6.3759078113000 \\
C94 & 5.8827883699000 & 10.5701587522000 & 8.4589315358000 \\
C95 & 6.6559562218000 & 9.7672361590000 & 4.8420863542000 \\
C96 & 4.3770532439000 & 10.2439124349000 & 5.0043437350000 \\
C97 & 4.8041978378000 & 10.5101170490000 & 9.3474673662000 \\
C98 & 7.1243320764000 & 10.9694811364000 & 8.9602131811000 \\
N99 & 5.4586906396000 & 9.9600570370000 & 4.2638343500000 \\
C100 & 4.9795722877000 & 10.8978710057000 & 10.6639485681000 \\
C101 & 7.2425134847000 & 11.3295352383000 & 10.2891518460000 \\
N102 & 6.1787792601000 & 11.3091238157000 & 11.1070574282000 \\
C103 & 5.4153982810000 & 11.4321506389000 & 2.3134205341000 \\
C104 & 6.6511826042000 & 12.0818292459000 & 2.2326471179000 \\
C105 & 4.2463823262000 & 12.1506593255000 & 2.0661331841000 \\
\hline
\end{tabular}



C107 $6.7120780196000 \quad 13.4511927985000 \quad 1.9889766052000$
$\begin{array}{llll}\text { C108 } & 4.3060440090000 & 13.5233294508000 & 1.8126596229000\end{array}$
$\begin{array}{lllll}\text { C109 } & 7.6340880345000 & 14.0233638925000 & 12.3960648833000\end{array}$
$\begin{array}{lllll}\text { C110 } & 5.2293134008000 & 14.1215106304000 & 12.2318715603000\end{array}$
$\begin{array}{lllll}\text { C111 } & 5.5332597443000 & 14.1851844407000 & 1.8114956972000\end{array}$
$\begin{array}{lllll}\text { C112 } & 7.7019559041000 & 15.3945144801000 & 12.1445391125000\end{array}$
C113 $5.2921398073000 \quad 15.489778998000011 .9770380403000$
$\begin{array}{llll}\text { C114 } & 5.5951777568000 & 15.7044593886000 & 1.7114763187000\end{array}$
$\begin{array}{lllll}\text { C115 } & 6.5379544687000 & 16.1233344184000 & 11.8987443592000\end{array}$
N116 $5.7555483206000 \quad 16.2504030536000 \quad 3.0941129717000$
C117 $4.6780518699000 \quad 16.3854192285000 \quad 3.8815835202000$
$\begin{array}{llll}\text { C118 } & 6.9865626856000 & 16.4974467811000 & 3.5643362163000\end{array}$

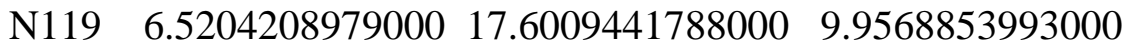
$\begin{array}{llll}\mathrm{C} 120 & 4.8071678517000 & 16.7431257159000 & 5.2079639173000\end{array}$
$\begin{array}{lllll}\text { C121 } & 7.1711668396000 & 16.8675950604000 & 4.8875050073000\end{array}$
$\begin{array}{llll}\text { C122 } & 7.6102764280000 & 17.4914862437000 & 9.1882357276000\end{array}$
C123 $5.2879991715000 \quad 17.6091787922000 \quad 9.4154344110000$
$\begin{array}{llll}\text { C124 } & 6.0771993349000 & 16.9723270494000 & 5.7517550330000\end{array}$
$\begin{array}{lllll}\text { C125 } & 7.5041411521000 & 17.3337738162000 & 7.8141057769000\end{array}$
$\begin{array}{llll}\text { C126 } & 5.1302388931000 & 17.4367214950000 & 8.0555275480000\end{array}$
$\begin{array}{lllll}\text { C127 } & 6.2445436369000 & 17.2607981396000 & 7.2194021236000\end{array}$
H128 $7.7938955944000 \quad 9.7277252522000 \quad 6.6254251029000$
H129 $3.5772681987000 \quad 10.6606584391000 \quad 6.9181689995000$

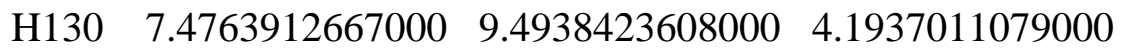
$\begin{array}{llll}H 131 & 3.4397127352000 & 10.3601064763000 & 4.4777596894000\end{array}$
H132 $3.8262528170000 \quad 10.16031756030009 .0476589663000$
H133 $8.0024277639000 \quad 11.0557008655000 \quad 8.3357737746000$ 

H134 $4.1726687479000 \quad 10.8760815631000 \quad 11.3827389809000$
H135 $8.1805286200000 \quad 11.6601973891000 \quad 10.7129009273000$
$\begin{array}{llll}\text { H136 } & 7.5694651977000 & 11.5252851655000 & 2.3840553298000\end{array}$
H137 $3.2837952853000 \quad 11.65184199080002 .0813051622000$
H138 $7.6734949653000 \quad 13.95588492730001 .9954768504000$
H139 $3.3887636154000 \quad 14.0730705521000 \quad 1.6347569006000$
$\begin{array}{lllll}\text { H140 } & 8.5468084061000 & 13.4660669422000 & 12.5749510882000\end{array}$
H141 $4.2632321035000 \quad 13.6327694814000 \quad 12.2778669258000$
H142 $8.6677847986000 \quad 15.8867002968000 \quad 12.1253745360000$
H143 $4.3743093575000 \quad 16.0471392080000 \quad 11.8104452949000$
H144 $6.4535067273000 \quad 16.0522840588000 \quad 1.1405098314000$
H145 $4.6774201493000 \quad 16.1181903067000 \quad 1.3001725399000$
H146 $3.7161028807000 \quad 16.16926033150003 .4364266896000$
$\begin{array}{llll}\mathrm{H} 147 & 7.8073486862000 & 16.3493125830000 & 2.8725994001000\end{array}$
H148 $3.8994259265000 \quad 16.8013877667000 \quad 5.7878259765000$
H149 $8.1846778916000 \quad 17.0549805482000 \quad 5.2112000455000$
H150 $8.5684778113000 \quad 17.5103785126000 \quad 9.6897286970000$
H151 $4.4626942357000 \quad 17.7559637613000 \quad 10.1057096771000$
$\begin{array}{llll}H 152 & 8.4242701953000 & 17.2371785500000 & 7.2572345514000\end{array}$
H153 $4.1234863309000 \quad 17.4610769804000 \quad 7.6688149154000$
$\begin{array}{llll}\mathrm{C} 154 & 5.3459305855000 & 9.9798678885000 & 2.7686975140000\end{array}$
$\begin{array}{llll}H 155 & 6.1667114836000 & 9.3840048329000 & 2.3774672578000\end{array}$
H156 $4.3985291090000 \quad 9.5137452505000 \quad 2.5094840077000$
$\begin{array}{llllll}\text { C157 } & 6.3260553107000 & 11.8570032202000 & 12.4962077523000\end{array}$
H158 $5.4633406194000 \quad 11.520104330500013 .0650353553000$
H159 $7.2327316109000 \quad 11.4277357554000 \quad 12.9161184957000$
$\begin{array}{lllll}\text { C160 } & 6.6387657249000 & 17.5752579580000 & 11.4475353186000\end{array}$ 


\begin{abstract}
H161 $5.8397146493000 \quad 18.2079320290000 \quad 11.8288517850000$
H162 $7.6023078275000 \quad 18.0073798335000 \quad 11.7071060065000$
\end{abstract}

$\mathbf{1}^{3+}$ CBPQT circling DNP (radical sandwiched by two DNP units)

$-3759.259517-3758.857425-3759.882275-3760.284367$

$\begin{array}{llll}\mathrm{N} 1 & 1.8833148865 & 9.2221504781 & 22.4295687531 \\ \mathrm{C} 2 & 3.1922568041 & 9.0456970558 & 22.1960152292 \\ \mathrm{C} 3 & 1.0384095839 & 9.5250135954 & 21.4287933660 \\ \mathrm{C} 4 & 1.3379370081 & 8.9478721702 & 23.7994505869 \\ \mathrm{C} 5 & 3.7032048937 & 9.1207385010 & 20.9112162230 \\ \mathrm{C} 6 & 1.4908747950 & 9.6193268006 & 20.1273755495 \\ \mathrm{C} 7 & 1.0746685682 & 7.4524272049 & 23.9038611853 \\ \mathrm{C} 8 & 2.8407078742 & 9.3803198093 & 19.8443807954 \\ \mathrm{C} 9 & -0.1198113643 & 6.9114521614 & 23.4221231222 \\ \mathrm{C} 10 & 2.0643989301 & 6.5976740441 & 24.3922625795 \\ \mathrm{C} 11 & 3.3320824468 & 9.3524995469 & 18.4320279963 \\ \mathrm{C} 12 & -0.2886653005 & 5.5288640067 & 23.3476714467 \\ \mathrm{C} 13 & 1.8953710210 & 5.2150243496 & 24.3186735040 \\ \mathrm{C} 14 & 2.4599590339 & 9.0240119625 & 17.3929657342 \\ \mathrm{C} 15 & 4.6735455159 & 9.6003534397 & 18.1201477403 \\ \mathrm{C} 16 & 0.7390663580 & 4.6720841139 & 23.7544575163 \\ \mathrm{C} 17 & 2.9516431064 & 8.9091568107 & 16.1057543654 \\ \mathrm{C} 18 & 5.1083666578 & 9.4575694062 & 16.8163480657 \\ \mathrm{C} 19 & 0.6361295426 & 3.1736109682 & 23.4911520081 \\ \mathrm{~N} 20 & 4.2522824946 & 9.1047351408 & 15.8420118581 \\ \mathrm{~N} 21 & 1.2062364436 & 2.8684454073 & 22.1632845156 \\ \mathrm{C} 22 & 4.7612033258 & 8.7977004790 & 14.4654065096\end{array}$




\begin{tabular}{|c|c|c|c|}
\hline $\mathrm{C} 23$ & 2.5283330486 & 2.5768761335 & 22.0159199166 \\
\hline $\mathrm{C} 24$ & 0.4303571080 & 3.0028397342 & 21.0519584605 \\
\hline $\mathrm{C} 25$ & 4.8340801498 & 7.2844464193 & 14.3145800788 \\
\hline $\mathrm{C} 26$ & 3.1027329512 & 2.4196593962 & 20.7898869546 \\
\hline $\mathrm{C} 27$ & 0.9442958679 & 2.8351646349 & 19.8005796235 \\
\hline $\mathrm{C} 28$ & 5.8538236854 & 6.5729552015 & 14.9523373837 \\
\hline C29 & 3.8460484933 & 6.5865684022 & 13.6198023256 \\
\hline $\mathrm{C} 30$ & 2.3345051976 & 2.5670070870 & 19.5891475527 \\
\hline $\mathrm{C} 31$ & 5.8473446743 & 5.1813155996 & 14.9619819809 \\
\hline $\mathrm{C} 32$ & 3.8504084939 & 5.1894233509 & 13.6116983252 \\
\hline $\mathrm{C} 33$ & 2.9156199372 & 2.5063378782 & 18.2907240548 \\
\hline C34 & 4.8291466111 & 4.4786816420 & 14.3084883887 \\
\hline $\mathrm{C} 35$ & 2.1421422230 & 2.6180447545 & 17.0898634009 \\
\hline $\mathrm{C} 36$ & 4.3257133683 & 2.3659238233 & 18.0829730245 \\
\hline $\mathrm{C} 37$ & 4.7353394023 & 2.9608455953 & 14.4456253134 \\
\hline $\mathrm{C} 38$ & 2.7477458210 & 2.6967137243 & 15.8735604780 \\
\hline C39 & 4.8775068420 & 2.4289785013 & 16.8389599862 \\
\hline N40 & 4.1037619099 & 2.6262529553 & 15.7350149130 \\
\hline $\mathrm{O} 41$ & -0.4147994241 & 5.7958676816 & 19.6436831344 \\
\hline $\mathrm{C} 42$ & 0.5774693454 & 5.8531572489 & 18.7193176769 \\
\hline $\mathrm{C} 43$ & 1.9063015492 & 5.9270206876 & 19.2615478692 \\
\hline $\mathrm{C} 44$ & 0.3777812460 & 5.8276954361 & 17.3602860832 \\
\hline $\mathrm{C} 45$ & 2.9958411534 & 5.9482834090 & 18.3691051512 \\
\hline $\mathrm{C} 46$ & 2.1280501713 & 5.9362591235 & 20.6687037444 \\
\hline $\mathrm{O} 47$ & -2.5355129100 & 4.5358668413 & 21.2497618833 \\
\hline $\mathrm{C} 48$ & 2.7773394378 & 5.9055980675 & 16.9646152457 \\
\hline C49 & 4.3262147832 & 6.0127858923 & 18.9076222705 \\
\hline
\end{tabular}




\begin{tabular}{llll} 
C50 & 3.4095688509 & 5.9657156064 & 21.1419288189 \\
O51 & 5.3154905228 & 6.0785132397 & 17.9795404521 \\
C52 & 4.5262800489 & 6.0187146887 & 20.2655917483 \\
C53 & -3.4779230218 & 2.5950298419 & 22.2443241218 \\
O54 & -2.2339532173 & 1.9410992361 & 22.4634966878 \\
C55 & -2.2634293656 & 0.5419276128 & 22.2102862228 \\
O56 & 7.6413746634 & 4.0341025617 & 17.8665800696 \\
C57 & -0.8456864524 & -0.0061861428 & 22.1606955380 \\
O58 & -0.2369140647 & 0.0955362880 & 23.4405686275 \\
C59 & 8.9796589530 & 2.1932004452 & 17.0990420060 \\
C60 & 0.6792950486 & -0.9378954893 & 23.7574315715 \\
O61 & 7.9426245080 & 1.3993558317 & 16.5539068945 \\
C62 & 2.0464668855 & -0.7728505877 & 23.0989793851 \\
C63 & 8.4161197458 & 0.5157407718 & 15.5531664298 \\
O64 & 1.8567729260 & -0.8420858664 & 21.6950946210 \\
C65 & 7.3611687131 & -0.5303379392 & 15.2469424126 \\
C66 & 2.9325924452 & -0.8101676934 & 20.8663955707 \\
O67 & 6.3120774270 & 0.0498279259 & 14.4880019966 \\
C68 & 2.5945703219 & -0.7885444658 & 19.4712349519 \\
C69 & 4.2413548577 & -0.8038851392 & 21.2728723493 \\
C70 & 5.3257724416 & -0.8919977295 & 14.0988441676 \\
C71 & 3.6350990780 & -0.7789119121 & 18.5231846343 \\
C72 & 4.0357262855 & -0.7129929158 & 14.8837319736 \\
C73 & 3.2832290064 & -0.7816694545 & 17.1310022333 \\
O74 & 4.33088333015 & -0.8345898760 & 16.2661496105 \\
\hline & 3.8171624287 & 8.8266036082 & 23.0511367399 \\
H75 & 0.0040968198 & 9.6945422235 & 21.6933309680
\end{tabular}




\begin{tabular}{llll} 
H77 & 2.0823372846 & 9.2867201854 & 24.5156092127 \\
H78 & 0.4312000221 & 9.5378315928 & 23.9070359721 \\
H79 & 4.7585284099 & 8.9360766015 & 20.7745369247 \\
H80 & 0.7802231875 & 9.8885832791 & 19.3580746844 \\
H81 & -0.9159925814 & 7.5671634802 & 23.0876071840 \\
H82 & 2.9759224182 & 7.0034553347 & 24.8174334940 \\
H83 & -1.2019425535 & 5.1161776864 & 22.9305618485 \\
H84 & 2.6804701620 & 4.5622622975 & 24.6838105436 \\
H85 & 1.4166001148 & 8.7944970587 & 17.5594206216 \\
H86 & 5.3915369846 & 9.9098752754 & 18.8668821196 \\
H87 & 2.3212180617 & 8.6340517784 & 15.2722423104 \\
H88 & 6.1362850826 & 9.6308401838 & 16.5293023073 \\
H89 & 1.1903976214 & 2.6017298537 & 24.2326618428 \\
H90 & -0.3979398670 & 2.8337997806 & 23.4784614915 \\
H91 & 4.0687945892 & 9.2470885397 & 13.7575502528 \\
H92 & 5.7363921273 & 9.2695240814 & 14.3735232406 \\
H93 & 3.1007797755 & 2.4907066243 & 22.9299700600 \\
H94 & -0.6080448307 & 3.2548361326 & 21.2288586292 \\
H95 & 4.1549733499 & 2.1826762892 & 20.7646620603 \\
H96 & 0.2672120273 & 2.9567556956 & 18.9675142825 \\
H97 & 6.6483327407 & 7.1088327921 & 15.4591180043 \\
H98 & 3.0681266716 & 7.1240966062 & 13.0890529304 \\
H99 & 6.6150902800 & 4.6455438517 & 15.5108607240 \\
H100 & 3.0742764242 & 4.65804855501 & 13.0724482810 \\
H101 & 1.0646160606 & 2.6664714713 & 17.1099679172 \\
\hline & 4.9987518073 & 2.1940682246 & 18.9085828403 \\
\hline 1.1276964460 & 2.5396608739 & 13.6453772217
\end{tabular}




\begin{tabular}{|c|c|c|c|}
\hline H104 & 5.7140706317 & 2.4842401152 & 14.4324574967 \\
\hline H105 & 2.1840128837 & 2.8194095778 & 14.9581181891 \\
\hline H106 & 5.9416293254 & 2.3139901715 & 16.6698611928 \\
\hline H107 & -0.6172721113 & 5.7666167503 & 16.9414006826 \\
\hline H108 & 1.2811047535 & 5.8948209228 & 21.3388900286 \\
\hline H109 & 3.6319368887 & 5.8929585144 & 16.3005988907 \\
\hline H110 & 3.5928075509 & 5.9408143613 & 22.2082822869 \\
\hline H111 & 5.5190585633 & 6.0488776979 & 20.6932985996 \\
\hline H112 & -4.2852653086 & 1.8656498813 & 22.1504859636 \\
\hline H113 & -3.6820496986 & 3.2407652208 & 23.0989293236 \\
\hline H114 & -2.8332554642 & 0.0325238041 & 22.9945463918 \\
\hline H115 & -2.7341156313 & 0.3413784371 & 21.2415686156 \\
\hline H116 & -0.2624267066 & 0.5442981508 & 21.4209427589 \\
\hline H117 & -0.9087006517 & -1.0565771725 & 21.858259511 \\
\hline H118 & 9.8007451739 & 1.5507559097 & 17.4322279411 \\
\hline H119 & 9.3624900554 & 2.8985879554 & 16.3557262945 \\
\hline H120 & 0.8224119587 & -0.9157493402 & 24.8374751191 \\
\hline 121 & 0.2632051548 & -1.9099278939 & 23.4774117839 \\
\hline H122 & 2.4926742399 & 0.1862329237 & 23.3666563631 \\
\hline H123 & 2.6959578012 & -1.5845804903 & 23.4369864485 \\
\hline H124 & 8.6727826757 & 1.0641270245 & 14.6421494433 \\
\hline H125 & 9.3095038439 & -0.0033820606 & 15.9146041704 \\
\hline H126 & 6.9742481868 & -0.9422694681 & 16.1797850846 \\
\hline H127 & 7.8329418007 & -1.3317398292 & 14.6704760224 \\
\hline H128 & 4.5008316780 & -0.8114968151 & 22.3229428417 \\
\hline H129 & 5.1009876847 & -0.7475661309 & 13.0407275435 \\
\hline H13 & 5.6945574696 & -1.9106356301 & 14.2384098474 \\
\hline
\end{tabular}




\begin{tabular}{llll} 
H131 & 3.5997877490 & 0.2697964030 & 14.6943536773 \\
H132 & 3.3336330154 & -1.4897957430 & 14.5698284296 \\
C133 & -2.6626305272 & 5.6680627623 & 20.4068698848 \\
H134 & -2.3905479198 & 6.5401918524 & 21.0012606505 \\
H135 & -3.6966026534 & 5.7797129606 & 20.0721365183 \\
C136 & -1.7483566555 & 5.6240359018 & 19.1870832247 \\
H137 & -2.0233939716 & 6.4408600396 & 18.5150754582 \\
H138 & -1.8452890251 & 4.6741367844 & 18.6540289128 \\
C139 & 6.6551048097 & 6.1533553924 & 18.4395547022 \\
H140 & 7.2540251198 & 6.4494518495 & 17.5817468993 \\
H141 & 6.7393728881 & 6.9241273203 & 19.2082843808 \\
C142 & 7.1980119925 & 4.8202943186 & 18.9576880416 \\
H143 & 6.4331975829 & 4.2806531714 & 19.5224951395 \\
H144 & 8.0399908129 & 5.0357624152 & 19.6239197319 \\
C145 & 8.4494003277 & 2.9533926628 & 18.3006892950 \\
H146 & 9.2969151541 & 3.3350230778 & 18.8787084128 \\
H147 & 7.8724228725 & 2.2673856177 & 18.9297204861 \\
C148 & 5.2768083716 & -0.7593166276 & 20.2951111697 \\
H149 & 6.3050066640 & -0.7348466055 & 20.6335879377 \\
C150 & 4.9937814172 & -0.7500094283 & 18.9570685363 \\
H151 & 5.7799418047 & -0.6898406939 & 18.2181011392 \\
C152 & 1.2344854775 & -0.7606483243 & 19.0509110437 \\
H153 & 0.4559431790 & -0.7687179746 & 19.7978412019 \\
C154 & 0.9389483435 & -0.7283437145 & 17.7178830988 \\
H155 & -0.0906261893 & -0.7077448791 & 17.3856696159 \\
\hline & 1.9678470141 & -0.7476053463 & 16.7397099685 \\
\hline
\end{tabular}




$\begin{array}{llll}\text { C158 } & 1.4982058557 & 5.8565002623 & 16.4845341440 \\ \text { H159 } & 1.3216374378 & 5.8121102955 & 15.4166348525 \\ \text { C160 } & -3.4222872188 & 3.4571328694 & 20.9874380091 \\ \text { H161 } & -4.4241922674 & 3.8377721985 & 20.7698671049 \\ \text { H162 } & -3.0732413800 & 2.8628513397 & 20.1376980545\end{array}$

$\mathbf{1}^{3+}$ CBPQT circling DNP (radical on the side)

-3759.269279-3758.889415 -3759.912428 -3760.292292

$\begin{array}{llll}\text { N1 } & 1.8261446986 & 9.2366701399 & 22.4422428625 \\ \text { C2 } & 3.1694865601 & 9.2462910382 & 22.2168130625 \\ \text { C3 } & 0.9772635100 & 9.2675283972 & 21.3765048941 \\ \text { C4 } & 1.2826000850 & 8.9907670604 & 23.7951555511 \\ \text { C5 } & 3.6890970409 & 9.2623555550 & 20.9561670635 \\ \text { C6 } & 1.4322300783 & 9.2758695046 & 20.0947569053 \\ \text { C7 } & 1.0172799086 & 7.4968146027 & 23.9378624276 \\ \text { C8 } & 2.8359309221 & 9.2421703384 & 19.8050229286 \\ \text { C9 } & -0.2023434114 & 6.9541504727 & 23.5281140776 \\ \text { C10 } & 2.0369613474 & 6.6406708177 & 24.3596561815 \\ \text { C11 } & 3.3351695643 & 9.1961833647 & 18.4725120307 \\ \text { C12 } & -0.3717389717 & 5.5715101258 & 23.4492759191 \\ \text { C13 } & 1.8682967923 & 5.2595287956 & 24.2906672766 \\ \text { C14 } & 2.4711849648 & 9.1091004913 & 17.3326171810 \\ \text { C15 } & 4.7361474003 & 9.2307333101 & 18.1665194235 \\ \text { C16 } & 0.6793649169 & 4.7198027657 & 23.7944011233 \\ \text { C17 } & 2.9764911072 & 9.0284853618 & 16.0706724738 \\ \text { C18 } & 5.1765910425 & 9.1503779596 & 16.8791864084 \\ \text { C19 } & 0.5624887932 & 3.2240510113 & 23.5509860427\end{array}$




\begin{tabular}{|c|c|c|c|}
\hline $\mathrm{N} 20$ & 4.3167481046 & 9.0446911242 & 15.8269776186 \\
\hline $\mathrm{N} 21$ & 1.1393814133 & 2.9209289204 & 22.2032555737 \\
\hline $\mathrm{C} 22$ & 4.8222009443 & 8.7467882162 & 14.4695052107 \\
\hline $\mathrm{C} 23$ & 2.4176966407 & 2.5290386494 & 22.0915149994 \\
\hline $\mathrm{C} 24$ & 0.3856957117 & 3.1406440330 & 21.1136467000 \\
\hline $\mathrm{C} 25$ & 4.8899240158 & 7.2334571766 & 14.2950939731 \\
\hline $\mathrm{C} 26$ & 3.0022397932 & 2.3436360968 & 20.8525229173 \\
\hline $\mathrm{C} 27$ & 0.9192678677 & 2.9580269308 & 19.8490415847 \\
\hline $\mathrm{C} 28$ & 5.9530372929 & 6.5207626489 & 14.8549139035 \\
\hline $\mathrm{C} 29$ & 3.8489776509 & 6.5326194820 & 13.6831378412 \\
\hline $\mathrm{C} 30$ & 2.2544955522 & 2.5811718959 & 19.6947411262 \\
\hline $\mathrm{C} 31$ & 5.9348296114 & 5.1291775535 & 14.8904696139 \\
\hline $\mathrm{C} 32$ & 3.8356857795 & 5.1368892738 & 13.7012893942 \\
\hline C33 & 2.8898976665 & 2.5354170406 & 18.3387818175 \\
\hline C34 & 4.8559779559 & 4.4306766444 & 14.3418215898 \\
\hline $\mathrm{C} 35$ & 2.1275932121 & 2.4509070026 & 17.1718498236 \\
\hline $\mathrm{C} 36$ & 4.2793070538 & 2.6438621608 & 18.2062950589 \\
\hline C37 & 4.7426951619 & 2.9243966219 & 14.5291040520 \\
\hline C38 & 2.7582336014 & 2.5425376572 & 15.9410268583 \\
\hline C39 & 4.8598460205 & 2.6951303752 & 16.9555672512 \\
\hline N40 & 4.0905336112 & 2.6686020040 & 15.8526114581 \\
\hline $\mathrm{O} 41$ & -0.4110878529 & 5.7291237645 & 19.6247864984 \\
\hline $\mathrm{C} 42$ & 0.5860120457 & 5.7927674390 & 18.7027510988 \\
\hline $\mathrm{C} 43$ & 1.9053117428 & 5.8775455834 & 19.2612698741 \\
\hline $\mathrm{C} 44$ & 0.3977497984 & 5.7293305625 & 17.3436344923 \\
\hline $\mathrm{C} 45$ & 3.0031240805 & 5.8664928677 & 18.3806504455 \\
\hline $\mathrm{C} 46$ & 2.1098138542 & 5.9440275968 & 20.6715233452 \\
\hline
\end{tabular}




\begin{tabular}{|c|c|c|c|}
\hline $\mathrm{O} 47$ & -2.4896447513 & 4.5617763252 & 21.2426147129 \\
\hline $\mathrm{C} 48$ & 2.8003679555 & 5.8151346116 & 16.9733310952 \\
\hline $\mathrm{C} 49$ & 4.3286851675 & 5.9205587229 & 18.9321844114 \\
\hline $\mathrm{C} 50$ & 3.3857505857 & 5.9926409678 & 21.1547393954 \\
\hline O51 & 5.3247344083 & 5.8898800849 & 18.0112037938 \\
\hline C52 & 4.5133632926 & 5.9849939450 & 20.2891172282 \\
\hline C53 & -3.3999175967 & 2.6503101980 & 22.3058944924 \\
\hline $\mathrm{O} 54$ & -2.1384089518 & 1.9990185141 & 22.4142491704 \\
\hline C55 & -2.1864157336 & 0.5956727354 & 22.2005724698 \\
\hline O56 & 7.5719046060 & 3.9979769744 & 17.9551883497 \\
\hline C57 & -0.7712769690 & 0.0498081639 & 22.0918062283 \\
\hline $\mathrm{O} 58$ & -0.0804973548 & 0.2125296978 & 23.3221637061 \\
\hline C59 & 8.9314162052 & 2.2123428682 & 17.1229025417 \\
\hline C60 & 0.7426708303 & -0.8772355034 & 23.7080448368 \\
\hline O61 & 7.8788870139 & 1.4833763923 & 16.5144296266 \\
\hline C62 & 2.1144768689 & -0.8405223318 & 23.0412698874 \\
\hline C63 & 8.3638679194 & 0.6539969222 & 15.4691545355 \\
\hline O64 & 1.8946674031 & -0.8667007311 & 21.6378301840 \\
\hline C65 & 7.3619795456 & -0.4394733801 & 15.1525188443 \\
\hline C66 & 2.9543243895 & -0.8103374546 & 20.7928068332 \\
\hline O67 & 6.2619935544 & 0.0944603292 & 14.4303983402 \\
\hline C68 & 2.5978196360 & -0.7737971769 & 19.4012588702 \\
\hline C69 & 4.2676288845 & -0.7809641751 & 21.1832052046 \\
\hline $\mathrm{C} 70$ & 5.3231440755 & -0.8999040481 & 14.0496983629 \\
\hline C71 & 3.6271177555 & -0.7108459916 & 18.4422062487 \\
\hline $\mathrm{C} 72$ & 4.0101866594 & -0.7235063161 & 14.7973316948 \\
\hline C73 & 3.2613127911 & -0.7194902291 & 17.0535429825 \\
\hline
\end{tabular}




\begin{tabular}{|c|c|c|c|}
\hline O74 & 4.3041662634 & -0.7015671259 & 16.1857922464 \\
\hline H75 & 3.8001636048 & 9.2410003967 & 23.0953906382 \\
\hline H76 & -0.0780502099 & 9.2968042665 & 21.6089283952 \\
\hline H77 & 2.0155463403 & 9.3394491660 & 24.5194310054 \\
\hline $\mathrm{H} 78$ & 0.3681022037 & 9.5713869591 & 23.9014327273 \\
\hline H79 & 4.7643292606 & 9.2718386806 & 20.8679778816 \\
\hline H80 & 0.6918788994 & 9.3167996255 & 19.3104531391 \\
\hline H81 & -1.0206070356 & 7.6088694160 & 23.2503685686 \\
\hline H82 & 2.9740033924 & 7.0487523423 & 24.7224389169 \\
\hline H83 & -1.3064091716 & 5.1620692446 & 23.0803274980 \\
\hline H84 & 2.6745579904 & 4.6061604008 & 24.6051041834 \\
\hline H85 & 1.3980367769 & 9.0734271571 & 17.4334274807 \\
\hline H86 & 5.4824196453 & 9.3410978173 & 18.9388257761 \\
\hline H87 & 2.3366658268 & 8.9392438385 & 15.2032831139 \\
\hline $\mathrm{H} 88$ & 6.2285908299 & 9.1881058661 & 16.6315813341 \\
\hline H89 & 1.1260311690 & 2.6431680862 & 24.2760754959 \\
\hline H90 & -0.4681517628 & 2.8768388884 & 23.5231965640 \\
\hline H91 & 4.1433511140 & 9.2004724151 & 13.7500214188 \\
\hline H92 & 5.8044571802 & 9.2051561883 & 14.3713232478 \\
\hline H93 & 2.9696048845 & 2.3948277848 & 23.0113768473 \\
\hline H94 & -0.6301654624 & 3.4846508426 & 21.2773850950 \\
\hline H95 & 4.0322077756 & 2.0192774659 & 20.8236187640 \\
\hline H96 & 0.2903559280 & 3.1802815969 & 18.9991687404 \\
\hline H97 & 6.7948231764 & 7.0548179378 & 15.2800702200 \\
\hline H98 & 3.0362924457 & 7.0695277596 & 13.2068508972 \\
\hline H99 & 6.7484661933 & 4.5973564655 & 15.3723786105 \\
\hline H100 & 3.0160804321 & 4.6036494247 & 13.2324742278 \\
\hline
\end{tabular}




\begin{tabular}{|c|c|c|c|}
\hline H101 & 1.0548819151 & 2.3266034654 & 17.1805332203 \\
\hline H102 & 4.9326659073 & 2.7190515002 & 19.0620313690 \\
\hline H103 & 4.1178787734 & 2.4732647165 & 13.7618203416 \\
\hline H104 & 5.7046879551 & 2.4164689949 & 14.5542153592 \\
\hline H105 & 2.2051991493 & 2.5284734624 & 15.0113968352 \\
\hline H106 & 5.9315979477 & 2.7711077526 & 16.8179659632 \\
\hline H107 & -0.5946551349 & 5.6607452235 & 16.9183656893 \\
\hline H108 & 1.2547060939 & 5.9725350929 & 21.3346877773 \\
\hline H109 & 3.6626595205 & 5.8404877743 & 16.3198420245 \\
\hline H110 & 3.5596710933 & 6.0481216720 & 22.2205528644 \\
\hline H111 & 5.5011546359 & 6.0369925913 & 20.7254658066 \\
\hline H112 & -4.2103993411 & 1.9198414633 & 22.2722766175 \\
\hline H113 & -3.5337063813 & 3.2876500592 & 23.1802764008 \\
\hline H114 & -2.7115834782 & 0.1079840438 & 23.0261460694 \\
\hline H115 & -2.7082280550 & 0.3745129192 & 21.2640751915 \\
\hline H116 & -0.2384039311 & 0.5716697107 & 21.2947105524 \\
\hline H117 & -0.8403846493 & -1.0093662021 & 21.8319969370 \\
\hline H118 & 9.7257236238 & 1.5268743513 & 17.4335165464 \\
\hline H119 & 9.3488118493 & 2.9443461261 & 16.4252974484 \\
\hline H120 & 0.8849840258 & -0.8100523390 & 24.7858456368 \\
\hline H121 & 0.2504186666 & -1.8243660339 & 23.4747141334 \\
\hline H122 & 2.6489550283 & 0.0698396478 & 23.3175632242 \\
\hline H123 & 2.6966516167 & -1.7102914230 & 23.3551505422 \\
\hline $\mathrm{H} 124$ & 8.5722507790 & 1.2456765058 & 14.5731464352 \\
\hline H125 & 9.2896213582 & 0.1668799233 & 15.7900531849 \\
\hline H126 & 7.0201644419 & -0.9009419706 & 16.0804509138 \\
\hline H127 & 7.8677614151 & -1.1948077580 & 14.5441947551 \\
\hline
\end{tabular}




\begin{tabular}{|c|c|c|c|}
\hline H128 & 4.5420850473 & -0.8191616405 & 22.2289421809 \\
\hline H129 & 5.1264593471 & -0.8182435007 & 12.9797927750 \\
\hline H130 & 5.7194623054 & -1.8956945431 & 14.2554213840 \\
\hline H131 & 3.5220995692 & 0.2109056296 & 14.5117195683 \\
\hline H132 & 3.3511922259 & -1.5602669083 & 14.5551164254 \\
\hline C133 & -2.6375945537 & 5.7067089738 & 20.4162968933 \\
\hline H134 & -2.3321220820 & 6.5695563626 & 21.0078380461 \\
\hline H135 & -3.6801035325 & 5.8305894643 & 20.1164346600 \\
\hline C136 & -1.7560833728 & 5.6480185002 & 19.1748353479 \\
\hline H137 & -1.9898677420 & 6.4951054429 & 18.5257123891 \\
\hline H138 & -1.9167306503 & 4.7172031880 & 18.6230693807 \\
\hline C139 & 6.6526401445 & 6.1524629664 & 18.4329901118 \\
\hline $\mathrm{H} 140$ & 7.1935240652 & 6.4624324745 & 17.5417824574 \\
\hline H141 & 6.6679866337 & 6.9677559077 & 19.1582948993 \\
\hline C142 & 7.3497419696 & 4.9238902703 & 19.0048268404 \\
\hline $\mathrm{H} 143$ & 6.7591920702 & 4.4594347291 & 19.7988685546 \\
\hline H144 & 8.3064423109 & 5.2476459487 & 19.4271029328 \\
\hline C145 & 8.4127519700 & 2.9277349662 & 18.3545305176 \\
\hline H146 & 9.2694872733 & 3.3158252156 & 18.9127661584 \\
\hline H147 & 7.8640492665 & 2.2233636247 & 18.9885183735 \\
\hline C148 & 5.2888272591 & -0.6818346112 & 20.1941948116 \\
\hline H149 & 6.3198925045 & -0.6405695747 & 20.5224750264 \\
\hline $\mathrm{C} 150$ & 4.9898796940 & -0.6417249838 & 18.8605072041 \\
\hline H151 & 5.7664316056 & -0.5422425575 & 18.1147796669 \\
\hline C152 & 1.2332346409 & -0.8002231108 & 18.9939190014 \\
\hline H153 & 0.4632973681 & -0.8612234554 & 19.7476305054 \\
\hline $\mathrm{C} 154$ & 0.9224378432 & -0.7773830316 & 17.6627173358 \\
\hline
\end{tabular}




$\begin{array}{llll}\text { H155 } & -0.1107888479 & -0.8064363885 & 17.3416639099 \\ \text { C156 } & 1.9414459588 & -0.7473221895 & 16.6736413072 \\ \text { H157 } & 1.6579449512 & -0.7467876146 & 15.6303665951 \\ \text { C158 } & 1.5269501331 & 5.7542371560 & 16.4784605475 \\ \text { H159 } & 1.3607528934 & 5.7228547163 & 15.4087365037 \\ \text { C160 } & -3.4381243191 & 3.5189848614 & 21.0542526703 \\ \text { H161 } & -4.4403975331 & 3.9381210535 & 20.9351607186 \\ \text { H162 } & -3.1903996768 & 2.9220112085 & 20.1711838678\end{array}$

\section{$\mathbf{1}^{2+}$ CBPQT circling DNP}

$-3759.403618-3759.208178-3760.237133-3760.432572$

$\begin{array}{llll}\mathrm{N} 1 & 1.7996182612 & 9.2291117089 & 22.3763443320 \\ \mathrm{C} 2 & 3.1435670458 & 9.2871244538 & 22.1630799791 \\ \mathrm{C} 3 & 0.9620680594 & 9.2169368828 & 21.3016859149 \\ \mathrm{C} 4 & 1.2529204954 & 8.9829592014 & 23.7288126977 \\ \mathrm{C} 5 & 3.6750480196 & 9.3226598668 & 20.9078251289 \\ \mathrm{C} 6 & 1.4292841940 & 9.2466800400 & 20.0245011702 \\ \mathrm{C} 7 & 1.0302597575 & 7.4858312765 & 23.8999839504 \\ \mathrm{C} 8 & 2.8354656461 & 9.2777388398 & 19.7472117573 \\ \text { C9 } & -0.1782590514 & 6.9024524848 & 23.5160641969 \\ \text { C10 } & 2.0766561786 & 6.6631258910 & 24.3238544879 \\ \text { C11 } & 3.3496837073 & 9.2595427155 & 18.4195272872 \\ \text { C12 } & -0.3097672705 & 5.5142041526 & 23.4628151587 \\ \text { C13 } & 1.9443974824 & 5.2767866310 & 24.2784975050 \\ \text { C14 } & 2.5029339062 & 9.1563241838 & 17.2685022525 \\ \text { C15 } & 4.7515365468 & 9.3294951398 & 18.1269589323 \\ \text { C16 } & 0.7667334609 & 4.69308688865 & 23.8042407514\end{array}$




\begin{tabular}{llll} 
C17 & 3.0273886594 & 9.0706263376 & 16.0150027523 \\
C18 & 5.2126933787 & 9.2386259306 & 16.8481250123 \\
C19 & 0.6929113631 & 3.1911956859 & 23.5569949808 \\
N20 & 4.3700747761 & 9.1030637455 & 15.7870562363 \\
N21 & 1.2372162844 & 2.8849046593 & 22.2183809430 \\
C22 & 4.8947350967 & 8.7750837940 & 14.4438850667 \\
C23 & 2.5402198203 & 2.5269721601 & 22.0514082475 \\
C24 & 0.4580479540 & 3.0823796578 & 21.1180637920 \\
C25 & 4.9196537950 & 7.2597742514 & 14.2791354221 \\
C26 & 3.0939723466 & 2.3618777382 & 20.8164758303 \\
C27 & 0.9498036227 & 2.9078534206 & 19.8592764765 \\
C28 & 5.9217617336 & 6.5153638937 & 14.9073566518 \\
C29 & 3.8972724106 & 6.5890920326 & 13.6080317634 \\
C30 & 2.3228215658 & 2.5751584308 & 19.6282163160 \\
C31 & 5.8654137056 & 5.1253834998 & 14.9348526389 \\
C32 & 3.8505755210 & 5.1929556788 & 13.6144626150 \\
C33 & 2.8885189338 & 2.5197938612 & 18.3230869217 \\
C34 & 4.8133445650 & 4.4541350840 & 14.3038623027 \\
C35 & 2.1038854716 & 2.6526138076 & 17.1317581688 \\
C36 & 4.2951844868 & 2.3678700203 & 18.1010613214 \\
C37 & 4.6716055502 & 2.9430099330 & 14.4546790775 \\
C38 & 2.6986920654 & 2.7231451831 & 15.9097889429 \\
C39 & 4.8352186056 & 2.42877966613 & 16.8517551702 \\
N40 & 4.0510483524 & 2.6290482483 & 15.7554425408 \\
O41 & -0.4594126124 & 5.7963095280 & 19.6834661172 \\
C43 & 0.5245926424 & 5.8525548866 & 18.7445912303 \\
\hline .8558517296 & 5.9226703358 & 19.2737782227
\end{tabular}




\begin{tabular}{llll} 
C44 & 0.3084926602 & 5.8288231683 & 17.3879342561 \\
C45 & 2.9348994316 & 5.9352641592 & 18.3695105347 \\
C46 & 2.0903367404 & 5.9600558937 & 20.6797728281 \\
O47 & -2.5365371075 & 4.5973627954 & 21.3558914464 \\
C48 & 2.7014214361 & 5.9127966400 & 16.9668595378 \\
C49 & 4.2712514734 & 5.9854003243 & 18.8946162686 \\
C50 & 3.3745389268 & 6.0079866611 & 21.1372911727 \\
O51 & 5.2557352425 & 6.0023442826 & 17.9577501191 \\
C52 & 4.4829809007 & 6.0238528442 & 20.2485009964 \\
C53 & -3.4149767662 & 2.6580282217 & 22.4084286848 \\
O54 & -2.1762463292 & 1.9826766332 & 22.5816504602 \\
C55 & -2.2032655394 & 0.6338630449 & 22.1404763523 \\
O56 & 7.6518501429 & 4.0643585356 & 17.8823223881 \\
C57 & -0.7957303916 & 0.0582994175 & 22.1145793973 \\
O58 & -0.2485134024 & 0.0438224868 & 23.4252555892 \\
C59 & 9.0073870539 & 2.2339871183 & 17.1396313024 \\
C60 & 0.6316580025 & -1.0323856624 & 23.6970462037 \\
O61 & 7.9778526879 & 1.4580162998 & 16.5560501377 \\
C62 & 2.0076421731 & -0.8865921638 & 23.0532998703 \\
C63 & 8.4669029050 & 0.5887240603 & 15.5514911307 \\
O64 & 1.8316242851 & -0.9508016701 & 21.6462881198 \\
C65 & 7.4269849601 & -0.4702785649 & 15.2373844251 \\
C66 & 2.9152268466 & -0.8929635786 & 20.8294150460 \\
O67 & 6.3689940859 & 0.0980196168 & 14.4816386685 \\
C68 & 2.5945735821 & -0.8010408642 & 19.4329947619 \\
C6 & 4.2187794795 & -0.9333114137 & 21.2504374360 \\
\hline 5.3916435827 & -0.8534836596 & 14.0920477247
\end{tabular}




\begin{tabular}{llll} 
C71 & 3.6461768050 & -0.7898867151 & 18.4971989924 \\
C72 & 4.0930061860 & -0.6749754377 & 14.8633110643 \\
C73 & 3.3125723361 & -0.7395344944 & 17.1012812897 \\
O74 & 4.3700940164 & -0.8021463894 & 16.2486609774 \\
H75 & 3.7656221714 & 9.3040714921 & 23.0476293849 \\
H76 & -0.0956227769 & 9.1888515791 & 21.5223293271 \\
H77 & 1.9691911383 & 9.3680255076 & 24.4515376914 \\
H78 & 0.3208261968 & 9.5382486686 & 23.8193965076 \\
H79 & 4.7504097361 & 9.3671344587 & 20.8305265876 \\
H80 & 0.6955101587 & 9.2416506107 & 19.2329907750 \\
H81 & -1.0161097451 & 7.5300753860 & 23.2331395134 \\
H82 & 3.0078282743 & 7.1026193638 & 24.6652387111 \\
H83 & -1.2346433561 & 5.0755010131 & 23.1037900857 \\
H84 & 2.7740438503 & 4.6501075246 & 24.5871506450 \\
H85 & 1.4294303218 & 9.0994280911 & 17.3555743381 \\
H86 & 5.4858486878 & 9.4545212173 & 18.9085636223 \\
H87 & 2.4010450225 & 8.9581366362 & 15.1413760440 \\
H88 & 6.2677596898 & 9.2859986738 & 16.6158741866 \\
H89 & 1.2814779533 & 2.6391201674 & 24.2872219085 \\
H90 & -0.3329263344 & 2.8283351389 & 23.5741873589 \\
H91 & 4.2493766635 & 9.2464086207 & 13.7048639383 \\
H92 & 5.8934810435 & 9.2010987202 & 14.3674705499 \\
H93 & 3.1148097419 & 2.3919919608 & 22.9580367019 \\
H94 & -0.5616109040 & 3.3960020225 & 21.3067654862 \\
H95 & 4.1304429201 & 2.0630957272 & 20.7726542096 \\
H96 & 0.2761852428 & 3.0923946773 & 19.0357177401 \\
\hline & 6.7430281040 & 7.0281817572 & 15.3943145171
\end{tabular}




\begin{tabular}{llll} 
H98 & 3.1269883562 & 7.1495655283 & 13.0898801329 \\
H99 & 6.6207906884 & 4.5685850116 & 15.4800688056 \\
H100 & 3.0466421665 & 4.6834717697 & 13.0947299589 \\
H101 & 1.0267269078 & 2.7180497600 & 17.1631856338 \\
H102 & 4.9759805241 & 2.2022221774 & 18.9217052763 \\
H103 & 4.0354691753 & 2.5385925250 & 13.6679659129 \\
H104 & 5.6338303044 & 2.4338145841 & 14.4265129011 \\
H105 & 2.1279575696 & 2.8587856929 & 15.0007844318 \\
H106 & 5.8983224062 & 2.3143024525 & 16.6737967124 \\
H107 & -0.6928995120 & 5.7715192533 & 16.9826974147 \\
H108 & 1.2487617263 & 5.9506402143 & 21.3593693326 \\
H109 & 3.5491438292 & 5.9263295357 & 16.2933426919 \\
H110 & 3.5690944199 & 6.0337619507 & 22.2004200966 \\
H111 & 5.4797290150 & 6.0652349165 & 20.6660621541 \\
H112 & -4.2395995594 & 1.9419498407 & 22.3753550298 \\
H113 & -3.5563750142 & 3.3185230304 & 23.2636881178 \\
H114 & -2.8349603849 & 0.0397996704 & 22.8074689624 \\
H115 & -2.6083635705 & 0.5730724579 & 21.1251955455 \\
H116 & -0.1624197081 & 0.6457301177 & 21.4494737990 \\
H117 & -0.8681876819 & -0.9626955839 & 21.7280378247 \\
H118 & 9.8122496615 & 1.5791711951 & 17.4882262323 \\
H119 & 9.4179155298 & 2.9444308539 & 16.4162022879 \\
H120 & 0.7679318041 & -1.0642453826 & 24.7774499850 \\
H121 & 0.1862967157 & -1.9765756420 & 23.3702168034 \\
H122 & 2.4638845939 & 0.0661560370 & 23.3272552827 \\
\hline & 2.6410741852 & -1.7087642456 & 23.3963616648 \\
\hline & 8.7202684908 & 1.1464769244 & 14.6452027802
\end{tabular}




\begin{tabular}{llll} 
H125 & 9.3656959014 & 0.0781501887 & 15.9118457532 \\
H126 & 7.0458388539 & -0.8909662234 & 16.1687482871 \\
H127 & 7.9100826886 & -1.2618979988 & 14.6569324577 \\
H128 & 4.4657430678 & -0.9916094705 & 22.3018965210 \\
H129 & 5.1755275454 & -0.7190368722 & 13.0307879665 \\
H130 & 5.7653220971 & -1.8685366317 & 14.2434300738 \\
H131 & 3.6622516760 & 0.3092094624 & 14.6705247909 \\
H132 & 3.3908791773 & -1.4481654806 & 14.5401550901 \\
C133 & -2.6870923144 & 5.7068128305 & 20.4867070119 \\
H134 & -2.3998609237 & 6.5931539463 & 21.0522144902 \\
H135 & -3.7296969117 & 5.8071576556 & 20.1747280484 \\
C136 & -1.7984682000 & 5.6350995345 & 19.2496306066 \\
H137 & -2.0801048149 & 6.4460731076 & 18.5727008271 \\
H138 & -1.9166883518 & 4.6796963669 & 18.7299264234 \\
C139 & 6.5889150892 & 6.1515872914 & 18.4062435690 \\
H140 & 7.1735471111 & 6.4439993932 & 17.5372559002 \\
H141 & 6.6461764767 & 6.9480977683 & 19.1505694328 \\
C142 & 7.1853890160 & 4.8568327419 & 18.9591297020 \\
H143 & 6.4396592590 & 4.3042285990 & 19.5366273304 \\
H144 & 8.0219523930 & 5.1158154687 & 19.6174236433 \\
C145 & 8.4496702440 & 2.9861805370 & 18.3332243280 \\
H146 & 9.2829903435 & 3.3653815871 & 18.9334802416 \\
H147 & 7.8591426364 & 2.2961095068 & 18.9452013084 \\
C148 & 5.26534655973 & -0.8800680386 & 20.2862774818 \\
H149 & 6.2899077156 & -0.8942079711 & 20.6368073733 \\
\hline & 4.9993065882 & -0.8147868188 & 18.9463210894 \\
\hline
\end{tabular}




$\begin{array}{llll}\text { C152 } & 1.2417472094 & -0.7065106354 & 18.9986646013 \\ \text { H153 } & 0.4549713895 & -0.7030429094 & 19.7371067824 \\ \text { C154 } & 0.9645950210 & -0.6178145145 & 17.6643680731 \\ \text { H155 } & -0.0593303078 & -0.5416998704 & 17.3219314135 \\ \text { C156 } & 2.0044067643 & -0.6489137994 & 16.6969389883 \\ \text { H157 } & 1.7448470291 & -0.5900118418 & 15.6495458168 \\ \text { C158 } & 1.4179988976 & 5.8710119054 & 16.4995177622 \\ \text { H159 } & 1.2316099703 & 5.8518840691 & 15.4322051725 \\ \text { C160 } & -3.4199628782 & 3.5071598344 & 21.1397094699 \\ \text { H161 } & -4.4346191339 & 3.8751460812 & 20.9619681861 \\ \text { H162 } & -3.1028303697 & 2.9107520752 & 20.2791451530\end{array}$

$\mathbf{1}^{1+}$ CBPQT circling DNP (radical sandwiched by two DNP units) -3759.517196 -3759.427362 -3760.466030 -3760.555865

$\begin{array}{llll}\mathrm{N} 1 & 1.7606967594 & 9.3434959343 & 22.4567685315 \\ \mathrm{C} 2 & 3.1289272074 & 9.3228305616 & 22.2295338565 \\ \mathrm{C} 3 & 0.9414990343 & 9.2393464335 & 21.3409306937 \\ \mathrm{C} 4 & 1.2234642723 & 8.9920071270 & 23.7712965179 \\ \mathrm{C} 5 & 3.6715899126 & 9.2827419560 & 21.0001673475 \\ \mathrm{C} 6 & 1.4113333000 & 9.1860474596 & 20.0840595800 \\ \text { C7 } & 1.0297030632 & 7.4838796907 & 23.9013789307 \\ \text { C8 } & 2.8519186739 & 9.2043129010 & 19.7792945763 \\ \text { C9 } & -0.1629140951 & 6.8880070326 & 23.4853361023 \\ \text { C10 } & 2.0743164088 & 6.6635839984 & 24.3346004593 \\ \text { C11 } & 3.3567877830 & 9.1717599726 & 18.5122390392 \\ \text { C12 } & -0.2830978709 & 5.5000630010 & 23.4178059162 \\ \text { C13 } & 1.9558410612 & 5.2759344840 & 24.2752792239\end{array}$




\begin{tabular}{|c|c|c|c|}
\hline C14 & 2.5230408853 & 9.0855725328 & 17.3033610745 \\
\hline $\mathrm{C} 15$ & 4.7916216260 & 9.2160892519 & 18.1826763440 \\
\hline $\mathrm{C} 16$ & 0.7917556964 & 4.6848168909 & 23.7782076542 \\
\hline $\mathrm{C} 17$ & 3.0439153559 & 9.0867430448 & 16.0663461014 \\
\hline $\mathrm{C} 18$ & 5.2411536930 & 9.2095301364 & 16.9141534242 \\
\hline C19 & 0.7276670681 & 3.1839983693 & 23.5321038978 \\
\hline N20 & 4.4029659779 & 9.2022088971 & 15.8097352208 \\
\hline N21 & 1.2734502056 & 2.8784509778 & 22.1945856963 \\
\hline $\mathrm{C} 22$ & 4.9102706175 & 8.8125695978 & 14.4933363786 \\
\hline $\mathrm{C} 23$ & 2.5861290621 & 2.5600659254 & 22.0253615987 \\
\hline $\mathrm{C} 24$ & 0.4869636673 & 3.0545631590 & 21.0961260133 \\
\hline $\mathrm{C} 25$ & 4.9184473901 & 7.2953152220 & 14.3183503679 \\
\hline $\mathrm{C} 26$ & 3.1400848248 & 2.4015907814 & 20.7900631319 \\
\hline $\mathrm{C} 27$ & 0.9778721960 & 2.8835060560 & 19.8371873075 \\
\hline $\mathrm{C} 28$ & 5.9179488075 & 6.5356334586 & 14.9316555240 \\
\hline $\mathrm{C} 29$ & 3.8960841363 & 6.6322187672 & 13.6378846028 \\
\hline $\mathrm{C} 30$ & 2.3569883090 & 2.5783331829 & 19.6040164834 \\
\hline $\mathrm{C} 31$ & 5.8625494797 & 5.1454490883 & 14.9346749749 \\
\hline C32 & 3.8470747908 & 5.2360424846 & 13.6196431169 \\
\hline C33 & 2.9167033725 & 2.5178390383 & 18.2970338237 \\
\hline C34 & 4.8114553873 & 4.4844302261 & 14.2924610733 \\
\hline $\mathrm{C} 35$ & 2.1235240323 & 2.6354555340 & 17.1106705678 \\
\hline C36 & 4.3236278363 & 2.3806869802 & 18.0664056459 \\
\hline $\mathrm{C} 37$ & 4.6715442233 & 2.9716549077 & 14.4215842582 \\
\hline $\mathrm{C} 38$ & 2.7095063507 & 2.7158674594 & 15.8852560917 \\
\hline C39 & 4.8540908886 & 2.4458482101 & 16.8134449889 \\
\hline N40 & 4.0613701598 & 2.6377239030 & 15.7221599864 \\
\hline
\end{tabular}




\begin{tabular}{|c|c|c|c|}
\hline O41 & -0.4762621751 & 5.7613323547 & 19.6347661111 \\
\hline $\mathrm{C} 42$ & 0.5100767005 & 5.8201078554 & 18.6962010154 \\
\hline $\mathrm{C} 43$ & 1.8386846612 & 5.8920694863 & 19.2280511099 \\
\hline $\mathrm{C} 44$ & 0.2942073681 & 5.7878191183 & 17.3398125823 \\
\hline $\mathrm{C} 45$ & 2.9189241452 & 5.8901890001 & 18.3268354226 \\
\hline $\mathrm{C} 46$ & 2.0684563320 & 5.9499165714 & 20.6328729543 \\
\hline $\mathrm{O} 47$ & -2.5489668437 & 4.5976394047 & 21.3109881823 \\
\hline $\mathrm{C} 48$ & 2.6866988021 & 5.8747189634 & 16.9248114175 \\
\hline $\mathrm{C} 49$ & 4.2534696516 & 5.9229049954 & 18.8558073151 \\
\hline $\mathrm{C} 50$ & 3.3501835171 & 5.9947037090 & 21.0937111312 \\
\hline O51 & 5.2441375047 & 5.8976063640 & 17.9251707812 \\
\hline C52 & 4.4609625152 & 5.9767300376 & 20.2089296825 \\
\hline C53 & -3.4345842768 & 2.6579339241 & 22.3489142986 \\
\hline O54 & -2.1945965853 & 1.9952893682 & 22.5605477177 \\
\hline C55 & -2.2077475251 & 0.6349305334 & 22.1553150361 \\
\hline O56 & 7.6671701107 & 4.0366871638 & 17.8701564668 \\
\hline $\mathrm{C} 57$ & -0.7953818229 & 0.0710289626 & 22.1439048283 \\
\hline O58 & -0.2476895758 & 0.0926112112 & 23.4541840035 \\
\hline C59 & 9.0209279364 & 2.2140704548 & 17.1195270390 \\
\hline C60 & 0.6586503139 & -0.9565037150 & 23.7453938569 \\
\hline O61 & 7.9801072786 & 1.4330410736 & 16.5637561146 \\
\hline C62 & 2.0279952952 & -0.8000554413 & 23.0896730227 \\
\hline C63 & 8.4449773961 & 0.5704743978 & 15.5429237574 \\
\hline O64 & 1.8472780084 & -0.9093385796 & 21.6862800562 \\
\hline C65 & 7.3903963055 & -0.4760830734 & 15.2348618059 \\
\hline C66 & 2.9243740376 & -0.8487327439 & 20.8604621034 \\
\hline O67 & 6.3317930222 & 0.1065869350 & 14.4911820480 \\
\hline
\end{tabular}




\begin{tabular}{|c|c|c|c|}
\hline C68 & 2.5915463157 & -0.7833086306 & 19.4654183148 \\
\hline C69 & 4.2312172099 & -0.8605704314 & 21.2716616546 \\
\hline $\mathrm{C} 70$ & 5.3449017066 & -0.8338800544 & 14.1004911486 \\
\hline C71 & 3.6352280146 & -0.7679002719 & 18.5207181009 \\
\hline $\mathrm{C} 72$ & 4.0529002918 & -0.6579118770 & 14.8838203170 \\
\hline C73 & 3.2894065791 & -0.7392532578 & 17.1273962695 \\
\hline $\mathrm{O} 74$ & 4.3402274108 & -0.7973747511 & 16.2661376406 \\
\hline $\mathrm{H} 75$ & 3.7425787892 & 9.3682671346 & 23.1205500655 \\
\hline H76 & -0.1201087975 & 9.2243969805 & 21.5501272432 \\
\hline $\mathrm{H} 77$ & 1.9183464874 & 9.3560473822 & 24.5281656583 \\
\hline $\mathrm{H} 78$ & 0.2701070353 & 9.5059386097 & 23.9014180546 \\
\hline H79 & 4.7497276247 & 9.2972530168 & 20.9340536970 \\
\hline H80 & 0.6842616388 & 9.1212465462 & 19.2885561018 \\
\hline H81 & -0.9995790210 & 7.5111194403 & 23.1895266448 \\
\hline H82 & 2.9942516532 & 7.1081263864 & 24.6995821641 \\
\hline H83 & -1.1977022958 & 5.0568523332 & 23.0377960359 \\
\hline H84 & 2.7845018644 & 4.6533650600 & 24.5955783416 \\
\hline H85 & 1.4522815098 & 8.9744355726 & 17.3851052490 \\
\hline H86 & 5.5312742367 & 9.2606405422 & 18.9694328324 \\
\hline H87 & 2.4185185850 & 8.9952880459 & 15.1880548655 \\
\hline H88 & 6.2995370933 & 9.2488695284 & 16.6883123949 \\
\hline H89 & 1.3178243191 & 2.6349182810 & 24.2635805715 \\
\hline H90 & -0.2962013460 & 2.8152524198 & 23.5490548282 \\
\hline H91 & 4.2796111551 & 9.2746389301 & 13.7329106065 \\
\hline H92 & 5.9200132067 & 9.2115479858 & 14.3913371819 \\
\hline H93 & 3.1683067831 & 2.4533886142 & 22.9310772220 \\
\hline H94 & -0.5377046722 & 3.3497943156 & 21.2868920457 \\
\hline
\end{tabular}




\begin{tabular}{llll} 
H95 & 4.1862717072 & 2.1399101200 & 20.7442994199 \\
H96 & 0.2984748570 & 3.0533725671 & 19.0153100275 \\
H97 & 6.7365419554 & 7.0414371015 & 15.4293227497 \\
H98 & 3.1244350477 & 7.2015704541 & 13.1310056444 \\
H99 & 6.6169049744 & 4.5794828207 & 15.4717595261 \\
H100 & 3.0402369508 & 4.7355064567 & 13.0951301514 \\
H101 & 1.0468495339 & 2.6954906243 & 17.1521582720 \\
H102 & 5.0126896167 & 2.2276783199 & 18.8824002150 \\
H103 & 4.0307040580 & 2.5754217468 & 13.6342169485 \\
H104 & 5.6341935009 & 2.4643440024 & 14.3808063880 \\
H105 & 2.1319966587 & 2.8520095390 & 14.9806047176 \\
H106 & 5.9173965292 & 2.3444905404 & 16.6309644148 \\
H107 & -0.7077756447 & 5.7280799713 & 16.9356901128 \\
H108 & 1.2251936653 & 5.9761904386 & 21.3089573353 \\
H109 & 3.5356559412 & 5.9035907297 & 16.2549145759 \\
H110 & 3.5383095648 & 6.0536718833 & 22.1564384435 \\
H111 & 5.4575873329 & 6.0143853104 & 20.6273390747 \\
H112 & -4.2514563066 & 1.9339484949 & 22.3002838977 \\
H113 & -3.6047398119 & 3.3260757453 & 23.1929028255 \\
H114 & -2.8349391429 & 0.0526610394 & 22.8368712368 \\
H115 & -2.6107601686 & 0.5440669385 & 21.1415585550 \\
H116 & -0.1678246127 & 0.6481341978 & 21.4646778167 \\
H117 & -0.8594789074 & -0.9598729681 & 21.7829074473 \\
H118 & 9.8353201508 & 1.5634206016 & 17.4536679908 \\
H119 & 9.4127284368 & 2.9208603570 & 16.3821388581 \\
H120 & 0.8028394664 & -0.9567711164 & 24.8253471255 \\
\hline & 0.2316747600 & -1.9183164692 & 23.4458256664
\end{tabular}




\begin{tabular}{llll} 
H122 & 2.4661673969 & 0.1690369250 & 23.3334828677 \\
H123 & 2.6795337096 & -1.5984101272 & 23.4542754488 \\
H124 & 8.6873319296 & 1.1351251478 & 14.6376819864 \\
H125 & 9.3459755100 & 0.0494099218 & 15.8825475514 \\
H126 & 7.0135043368 & -0.8966562584 & 16.1680760651 \\
H127 & 7.8591417792 & -1.2706326733 & 14.6466205081 \\
H128 & 4.4872124363 & -0.8953624993 & 22.3221373924 \\
H129 & 5.1199656149 & -0.6860073117 & 13.0428031400 \\
H130 & 5.7140065720 & -1.8527894334 & 14.2365840725 \\
H131 & 3.6223052139 & 0.3283874329 & 14.7029849116 \\
H132 & 3.3481903552 & -1.4280108772 & 14.5588131663 \\
C133 & -2.6959406926 & 5.7086146240 & 20.4426520875 \\
H134 & -2.3934866922 & 6.5904774419 & 21.0069797005 \\
H135 & -3.7405925782 & 5.8191663908 & 20.1400757294 \\
C136 & -1.8149149614 & 5.6283673254 & 19.2012245008 \\
H137 & -2.0843254335 & 6.4484125128 & 18.5296737708 \\
H138 & -1.9539825142 & 4.6787522958 & 18.6754475167 \\
C139 & 6.5664130529 & 6.1052842509 & 18.3732874014 \\
H140 & 7.1454705605 & 6.4070456640 & 17.5037130851 \\
H141 & 6.5933817783 & 6.9128363982 & 19.1063278574 \\
C142 & 7.2063922019 & 4.8395298115 & 18.9423168013 \\
H143 & 6.4861560322 & 4.2818587993 & 19.5465370152 \\
H144 & 8.0517792749 & 5.1305642960 & 19.5761573256 \\
C145 & 8.4858275757 & 2.9745890551 & 18.3182509934 \\
H146 & 9.3295301116 & 3.3699651030 & 18.8933296044 \\
\hline & 7.9170058844 & 2.2870164546 & 18.9529488822 \\
\hline
\end{tabular}




$\begin{array}{llll}\text { H149 } & 6.2963689162 & -0.7904193257 & 20.6408405219 \\ \text { C150 } & 4.9920397272 & -0.7613866673 & 18.9596024948 \\ \text { H151 } & 5.7813272441 & -0.6897572364 & 18.2249574751 \\ \text { C152 } & 1.2342589964 & -0.7125804195 & 19.0414182685 \\ \text { H153 } & 0.4539596076 & -0.7098725015 & 19.7864819927 \\ \text { C154 } & 0.9447642430 & -0.6417563526 & 17.7086604205 \\ \text { H155 } & -0.0830882095 & -0.5797106828 & 17.3746710794 \\ \text { C156 } & 1.9768367114 & -0.6706309354 & 16.7331213973 \\ \text { H157 } & 1.7083424506 & -0.6247868999 & 15.6873078068 \\ \text { C158 } & 1.4049633260 & 5.8329630464 & 16.4536214242 \\ \text { H159 } & 1.2224817863 & 5.8232122938 & 15.3854493745 \\ \text { C160 } & -3.4121839186 & 3.4974328655 & 21.0740172182 \\ \text { H161 } & -4.4256585434 & 3.8520427659 & 20.8637511951 \\ \text { H162 } & -3.0633339164 & 2.8981215849 & 20.2274620675\end{array}$

$\mathbf{1}^{1+}$ CBPQT circling DNP (radical on the side)

$\begin{array}{lrrr}-3759.501530 & -3759.410095 & -3760.449638 & -3760.541073 \\ \mathrm{~N} 1 & 1.7933352820 & 9.2324558401 & 22.5005931498 \\ \mathrm{C} 2 & 3.1327478036 & 9.2967117293 & 22.2618677949 \\ \mathrm{C} 3 & 0.9373013429 & 9.2178540000 & 21.4405574166 \\ \mathrm{C} 4 & 1.2725471677 & 8.9646136104 & 23.8604733693 \\ \mathrm{C} 5 & 3.6411581137 & 9.3416638362 & 20.9974013522 \\ \mathrm{C} 6 & 1.3808603004 & 9.2564969710 & 20.1554197032 \\ \mathrm{C} 7 & 1.0536985383 & 7.4656930230 & 24.0131399016 \\ \text { C8 } & 2.7809168996 & 9.2979955989 & 19.8520154775 \\ \text { C9 } & -0.1564290866 & 6.8871642809 & 23.6277224942 \\ \text { C10 } & 2.1085599790 & 6.6366206071 & 24.4007715779\end{array}$




\begin{tabular}{|c|c|c|c|}
\hline C11 & 3.2678053772 & 9.2824652594 & 18.5143460490 \\
\hline C12 & -0.2813771785 & 5.5007407351 & 23.5358988635 \\
\hline $\mathrm{C} 13$ & 1.9803102958 & 5.2514123425 & 24.3180480714 \\
\hline C14 & 2.3978114931 & 9.1676729331 & 17.3821880709 \\
\hline $\mathrm{C} 15$ & 4.6624790647 & 9.3601611878 & 18.1906690045 \\
\hline $\mathrm{C} 16$ & 0.8019796077 & 4.6725104357 & 23.8383527908 \\
\hline $\mathrm{C} 17$ & 2.8968425793 & 9.0676102292 & 16.1190690128 \\
\hline C18 & 5.0970753659 & 9.2593962821 & 16.9038528255 \\
\hline C19 & 0.7269520548 & 3.1749793026 & 23.5437346950 \\
\hline $\mathrm{N} 20$ & 4.2336541730 & 9.1052418325 & 15.8621163743 \\
\hline $\mathrm{N} 21$ & 1.2490066297 & 2.8612384457 & 22.2195190208 \\
\hline $\mathrm{C} 22$ & 4.7344060351 & 8.7640305250 & 14.5130985520 \\
\hline $\mathrm{C} 23$ & 2.5785039925 & 2.5379218735 & 22.0237689420 \\
\hline $\mathrm{C} 24$ & 0.4763966888 & 3.0919624222 & 21.0930227966 \\
\hline $\mathrm{C} 25$ & 4.7840427805 & 7.2490646732 & 14.3564931302 \\
\hline $\mathrm{C} 26$ & 3.1294218768 & 2.3995351264 & 20.8038194195 \\
\hline $\mathrm{C} 27$ & 0.9542410899 & 2.9500040243 & 19.8431855835 \\
\hline $\mathrm{C} 28$ & 5.7817072890 & 6.5218483385 & 15.0109542246 \\
\hline C29 & 3.7993233015 & 6.5583288958 & 13.6518539690 \\
\hline $\mathrm{C} 30$ & 2.3621486550 & 2.6200819822 & 19.5700590572 \\
\hline $\mathrm{C} 31$ & 5.7639414395 & 5.1308884893 & 15.0208119494 \\
\hline $\mathrm{C} 32$ & 3.7979524107 & 5.1609823678 & 13.6359909759 \\
\hline $\mathrm{C} 33$ & 2.9037255918 & 2.5444255026 & 18.3212446626 \\
\hline C34 & 4.7610623615 & 4.4340191480 & 14.3390522388 \\
\hline $\mathrm{C} 35$ & 2.1355649582 & 2.7527443009 & 17.0880712423 \\
\hline $\mathrm{C} 36$ & 4.3231756693 & 2.2698555879 & 18.0504523209 \\
\hline C37 & 4.6803243221 & 2.9104161234 & 14.4345464052 \\
\hline
\end{tabular}




\begin{tabular}{|c|c|c|c|}
\hline C38 & 2.7092588072 & 2.7377813298 & 15.8763349832 \\
\hline C39 & 4.8396356056 & 2.2677938199 & 16.8091181539 \\
\hline N40 & 4.0591901177 & 2.4778391387 & 15.6785711762 \\
\hline $\mathrm{O} 41$ & -0.5465625799 & 5.9670174000 & 19.8409141310 \\
\hline $\mathrm{C} 42$ & 0.4197879002 & 5.9753559477 & 18.8800734935 \\
\hline $\mathrm{C} 43$ & 1.7662623590 & 6.0018306673 & 19.3692193865 \\
\hline $\mathrm{C} 44$ & 0.1647556614 & 5.9594236566 & 17.5295134198 \\
\hline $\mathrm{C} 45$ & 2.8173547836 & 5.9902954014 & 18.4334032513 \\
\hline C46 & 2.0455912188 & 6.0151518966 & 20.7669892783 \\
\hline $\mathrm{O} 47$ & -2.6313437936 & 4.6798214240 & 21.5177940019 \\
\hline $\mathrm{C} 48$ & 2.5434297629 & 5.9583407492 & 17.0396383520 \\
\hline $\mathrm{C} 49$ & 4.1687484142 & 6.0156373609 & 18.9186904140 \\
\hline $\mathrm{C} 50$ & 3.3418267605 & 6.0267688416 & 21.1864459825 \\
\hline O51 & 5.1292887125 & 6.0411503462 & 17.9557111670 \\
\hline C52 & 4.4230385120 & 6.0334712211 & 20.2646237604 \\
\hline C53 & -3.4688913209 & 2.6420957864 & 22.4108513465 \\
\hline O54 & -2.2337052459 & 1.9759762183 & 22.6234221662 \\
\hline C55 & -2.1852622450 & 0.6888950429 & 22.0289842672 \\
\hline O56 & 7.6458782973 & 4.0296465063 & 17.8814957243 \\
\hline C57 & -0.7760634275 & 0.1183613941 & 22.0830935554 \\
\hline O58 & -0.3600556970 & -0.0337319147 & 23.4337874017 \\
\hline C59 & 9.0160601400 & 2.1647703934 & 17.2303494478 \\
\hline C60 & 0.5961072017 & -1.0552194900 & 23.6457471182 \\
\hline O61 & 8.0446126329 & 1.3667354475 & 16.5860814279 \\
\hline C62 & 1.9716091371 & -0.7925545548 & 23.0336146300 \\
\hline C63 & 8.5935806628 & 0.4642822331 & 15.6481970282 \\
\hline O64 & 1.8458051720 & -0.8800419930 & 21.6238969551 \\
\hline
\end{tabular}




\begin{tabular}{llll} 
C65 & 7.5566581289 & -0.5913728581 & 15.3080276147 \\
C66 & 2.9526183744 & -0.8337404162 & 20.8360824740 \\
O67 & 6.5611009565 & -0.0329577776 & 14.4672929616 \\
C68 & 2.6665808498 & -0.7532516575 & 19.4321951318 \\
C69 & 4.2452585165 & -0.9010103215 & 21.2876972836 \\
C70 & 5.5428442927 & -0.9562278274 & 14.1211537066 \\
C71 & 3.7361898221 & -0.7960985427 & 18.5189732999 \\
C72 & 4.2551413699 & -0.72049866191 & 14.8954512504 \\
C73 & 3.4344195200 & -0.7650440513 & 17.1152641576 \\
O74 & 4.5038023423 & -0.8953162270 & 16.2818166161 \\
H75 & 3.7708980941 & 9.3086131956 & 23.1349169947 \\
H76 & -0.1157515881 & 9.1751951342 & 21.6798280202 \\
H77 & 2.0032466635 & 9.3409210785 & 24.5736022842 \\
H78 & 0.3426515627 & 9.5192513436 & 23.9770545165 \\
H79 & 4.7146954603 & 9.3872356800 & 20.9000629626 \\
H80 & 0.6335092721 & 9.2413046654 & 19.3769426412 \\
H81 & -0.9990114840 & 7.5194989868 & 23.3694700199 \\
H82 & 3.0426369117 & 7.0721629807 & 24.7402872753 \\
H83 & -1.2065178477 & 5.0665069804 & 23.1720485317 \\
H84 & 2.8167977183 & 4.6194048131 & 24.5965944118 \\
H85 & 1.3268125838 & 9.0986417184 & 17.4926031480 \\
H86 & 5.4125283619 & 9.4962630422 & 18.9545698879 \\
H87 & 2.2533498474 & 8.9338531672 & 15.2609428398 \\
H88 & 6.1466636732 & 9.3117484997 & 16.6491229138 \\
H89 & 1.3082232442 & 2.6156260694 & 24.2775374735 \\
H91 & -0.3061635937 & 2.8317267556 & 23.5818824165 \\
\hline .0668166555 & 9.2172523105 & 13.7822497432 \\
Ho
\end{tabular}




\begin{tabular}{llll} 
H92 & 5.7239228138 & 9.2065853356 & 14.4112688589 \\
H93 & 3.1541515202 & 2.3897278261 & 22.9297546945 \\
H94 & -0.5460377325 & 3.3885408090 & 21.2940186450 \\
H95 & 4.1686464290 & 2.1100568771 & 20.7542997397 \\
H96 & 0.2701802554 & 3.1391330567 & 19.0289260834 \\
H97 & 6.5685048195 & 7.0516384520 & 15.5349048854 \\
H98 & 3.0273472030 & 7.1044053108 & 13.1202044374 \\
H99 & 6.5108569877 & 4.5834268039 & 15.5874937101 \\
H100 & 3.0276417676 & 4.6362756329 & 13.0810570602 \\
H101 & 1.0736103222 & 2.9432434239 & 17.1359179556 \\
H102 & 4.9928087048 & 2.0206638469 & 18.8611076691 \\
H103 & 4.0932691969 & 2.5269308692 & 13.5962000511 \\
H104 & 5.6726825138 & 2.4616485923 & 14.3941548631 \\
H105 & 2.1403782149 & 2.9128003711 & 14.9709393838 \\
H106 & 5.8846854918 & 2.0532607118 & 16.6185388150 \\
H107 & -0.8494929860 & 5.9339134578 & 17.1543235903 \\
H108 & 1.2255158638 & 5.9952016754 & 21.4704109785 \\
H109 & 3.3705715713 & 5.9202270082 & 16.3429556028 \\
H110 & 3.5659765609 & 6.0218931379 & 22.2441650855 \\
H111 & 5.4342307278 & 6.0406580540 & 20.6489962295 \\
H112 & -4.2785987926 & 1.9158349164 & 22.3004266599 \\
H113 & -3.6664299730 & 3.2572053453 & 23.2887292405 \\
H114 & -2.8774880537 & 0.0195187298 & 22.5497460024 \\
H115 & -2.4773538825 & 0.7430354156 & 20.9751521919 \\
H116 & -0.0832341177 & 0.7631135572 & 21.5438870585 \\
-0.8109398026 & -0.8618029568 & 21.5959342004 \\
\hline & 9.8070378997 & 1.5278974212 & 17.6399821692
\end{tabular}




\begin{tabular}{llll} 
H119 & 9.4656014210 & 2.8786023365 & 16.5333732116 \\
H120 & 0.7216113638 & -1.1444987446 & 24.7246329210 \\
H121 & 0.2231045879 & -2.0073479929 & 23.2547030838 \\
H122 & 2.3447326768 & 0.1938956594 & 23.3116600216 \\
H123 & 2.6531108109 & -1.5639860889 & 23.4027361396 \\
H124 & 8.9095667964 & 0.9863447654 & 14.7399606854 \\
H125 & 9.4621514861 & -0.0423897658 & 16.0822811544 \\
H126 & 7.1101367129 & -0.9630316158 & 16.2306402393 \\
H127 & 8.0560158567 & -1.4165274743 & 14.7902296648 \\
H128 & 4.4671245864 & -0.9422208210 & 22.3455938065 \\
H129 & 5.3159715524 & -0.8324036356 & 13.0606283677 \\
H130 & 5.8861908812 & -1.9814707561 & 14.2819991867 \\
H131 & 3.8857906362 & 0.2928316136 & 14.7312227578 \\
H132 & 3.5183825084 & -1.4504366129 & 14.5476192151 \\
C133 & -2.7667563953 & 5.8074108890 & 20.6729946957 \\
H134 & -2.4726524257 & 6.6771131307 & 21.2607284082 \\
H135 & -3.8098068266 & 5.9245971623 & 20.3649431267 \\
C136 & -1.8844014957 & 5.7648341958 & 19.4297199115 \\
H137 & -2.2026501478 & 6.5692827851 & 18.7599814681 \\
H138 & -1.9804352050 & 4.8092240464 & 18.9060524093 \\
C139 & 6.4744133487 & 6.0718064128 & 18.3849128015 \\
H140 & 7.0727956819 & 6.3224523412 & 17.5123694413 \\
H141 & 6.6062186450 & 6.8577416422 & 19.1313226822 \\
H142 & 6.9642510718 & 4.7172345422 & 18.9176263692 \\
H143 & 6.1137568107 & 4.1245004513 & 19.2627516713 \\
\hline & 7.6526948530 & 4.8864720782 & 19.7536943070 \\
H & &
\end{tabular}




$\begin{array}{llll}\text { H146 } & 9.1258914195 & 3.2458392179 & 19.0807449417 \\ \text { H147 } & 7.6794421581 & 2.2214355016 & 18.8945960178 \\ \text { C148 } & 5.3127112749 & -0.8921972513 & 20.3458587692 \\ \text { H149 } & 6.3297359427 & -0.9212333273 & 20.7179254517 \\ \text { C150 } & 5.0769765104 & -0.8482940430 & 18.9987947121 \\ \text { H151 } & 5.8894444570 & -0.8138428348 & 18.2869538724 \\ \text { C152 } & 1.3271003519 & -0.6203790878 & 18.9689845518 \\ \text { H153 } & 0.5291743747 & -0.5499895859 & 19.6919003032 \\ \text { C154 } & 1.0820814690 & -0.5528524945 & 17.6291208024 \\ \text { H155 } & 0.0690141565 & -0.4356773275 & 17.2649151073 \\ \text { C156 } & 2.1393337868 & -0.6379369191 & 16.6841867450 \\ \text { H157 } & 1.9029027950 & -0.5805500479 & 15.6318072214 \\ \text { C158 } & 1.2471384273 & 5.9533251978 & 16.6092748302 \\ \text { H159 } & 1.0315374016 & 5.9186304739 & 15.5477661232 \\ \text { C160 } & -3.4332728871 & 3.5576680913 & 21.1878955109 \\ \text { H161 } & -4.4537390280 & 3.8821548665 & 20.9606945009 \\ \text { H162 } & -3.0300403417 & 3.0253560508 & 20.3214638023\end{array}$

\begin{tabular}{llll}
\multicolumn{4}{l}{$\mathbf{1}^{0} \mathrm{CBPQT}$ circling DNP } \\
-3759.603965 & -3759.552467 & -3760.600228 & -3760.651725 \\
$\mathrm{C} 1$ & 6.0672550828 & 10.5154485022 & 6.8900874181 \\
$\mathrm{C} 2$ & 7.1486915595 & 10.5587093796 & 5.9060296160 \\
$\mathrm{C} 3$ & 4.7754581064 & 10.2125835577 & 6.2742468111 \\
$\mathrm{C} 4$ & 6.2533672010 & 10.7435417045 & 8.2332464645 \\
$\mathrm{C} 5$ & 6.9437585586 & 10.3363454755 & 4.5944170718 \\
$\mathrm{C} 6$ & 4.6331278756 & 10.0004913722 & 4.9510763679 \\
C7 & 5.1775511915 & 10.7654773808 & 9.2233895044
\end{tabular}




\begin{tabular}{|c|c|c|c|}
\hline $\mathrm{C} 8$ & 7.5547246523 & 11.0277461215 & 8.8358391344 \\
\hline N9 & 5.7052818467 & 9.9961892723 & 4.0633824871 \\
\hline $\mathrm{C} 10$ & 5.3996436487 & 10.9894908552 & 10.5348570846 \\
\hline $\mathrm{C} 11$ & 7.7152568088 & 11.2363977930 & 10.1560265121 \\
\hline N12 & 6.6705708641 & 11.1588072436 & 11.0715319688 \\
\hline $\mathrm{C} 13$ & 5.3082753369 & 11.6669542414 & 2.2756067703 \\
\hline $\mathrm{C} 14$ & 6.4182791977 & 12.4556182097 & 1.9636490819 \\
\hline $\mathrm{C} 15$ & 4.0537658442 & 12.2776606604 & 2.3538517581 \\
\hline $\mathrm{C} 16$ & 6.6882699847 & 13.2926331855 & 12.3383055053 \\
\hline $\mathrm{C} 17$ & 6.2896416819 & 13.8304988435 & 1.7824682854 \\
\hline $\mathrm{C} 18$ & 3.9218159430 & 13.6506504108 & 2.1643494383 \\
\hline C19 & 7.8016631173 & 14.1272705975 & 12.2186126935 \\
\hline $\mathrm{C} 20$ & 5.4161634992 & 13.8680954320 & 12.3104581452 \\
\hline $\mathrm{C} 21$ & 5.0410422806 & 14.4444872906 & 1.8990284727 \\
\hline $\mathrm{C} 22$ & 7.6388583963 & 15.5001033647 & 12.0292184976 \\
\hline $\mathrm{C} 23$ & 5.2501749840 & 15.2321500585 & 12.0968214653 \\
\hline $\mathrm{C} 24$ & 4.9262587918 & 15.9550515727 & 1.8544026038 \\
\hline $\mathrm{C} 25$ & 6.3626631441 & 16.0626489426 & 11.9382531969 \\
\hline N26 & 5.0346949529 & 16.5609476737 & 3.1749366638 \\
\hline $\mathrm{C} 27$ & 3.9449723218 & 16.5669903356 & 4.0417771376 \\
\hline $\mathrm{C} 28$ & 6.2955244562 & 16.6874596743 & 3.7501797921 \\
\hline N29 & 5.9319593704 & 17.7444284693 & 10.1677353024 \\
\hline $\mathrm{C} 30$ & 4.0657226057 & 16.7985877660 & 5.3627836588 \\
\hline $\mathrm{C} 31$ & 6.4718066608 & 16.9187604248 & 5.0627529603 \\
\hline $\mathrm{C} 32$ & 6.9955022288 & 17.6462146564 & 9.2834706016 \\
\hline C33 & 4.6581695949 & 17.5839856639 & 9.6369509269 \\
\hline C34 & 5.3588783541 & 17.0125831480 & 6.0065353038 \\
\hline
\end{tabular}




\begin{tabular}{|c|c|c|c|}
\hline $\mathrm{C} 35$ & 6.8359887629 & 17.4501838070 & 7.9619892231 \\
\hline C36 & 4.4437683480 & 17.3748626957 & 8.3212913107 \\
\hline C37 & 5.5275449153 & 17.2614616540 & 7.3476025194 \\
\hline $\mathrm{O} 38$ & 8.3014899458 & 13.6722792093 & 4.8573534857 \\
\hline C39 & 9.6763485585 & 13.5402019976 & 4.5766593687 \\
\hline $\mathrm{C} 40$ & 7.9478477435 & 13.9097853281 & 6.1553050368 \\
\hline $\mathrm{C} 41$ & 9.8293310066 & 12.8691642061 & 3.2257063429 \\
\hline $\mathrm{C} 42$ & 6.5485861767 & 13.8285639295 & 6.4283186383 \\
\hline $\mathrm{C} 43$ & 8.8478009749 & 14.1933120799 & 7.1619353829 \\
\hline $\mathrm{O} 44$ & 9.3840079039 & 13.6340690423 & 2.1266166360 \\
\hline $\mathrm{C} 45$ & 5.6031558625 & 13.5600828805 & 5.4043509158 \\
\hline $\mathrm{C} 46$ & 6.1169685631 & 14.0093887829 & 7.7635554945 \\
\hline $\mathrm{C} 47$ & 8.3841667984 & 14.3786778372 & 8.4845992661 \\
\hline $\mathrm{C} 48$ & 10.3354945005 & 14.5647996860 & 1.6519150913 \\
\hline $\mathrm{C} 49$ & 4.2765313001 & 13.4818640608 & 5.7177181828 \\
\hline $\mathrm{C} 50$ & 4.7195326272 & 13.8902044570 & 8.0528623806 \\
\hline C51 & 7.0524088969 & 14.2807279851 & 8.7872365411 \\
\hline C52 & 9.8111331498 & 15.1829668543 & 0.3664864070 \\
\hline C53 & 3.8164352193 & 13.6439600180 & 7.0464226636 \\
\hline O54 & 4.3955879326 & 14.0171491977 & 9.3695747959 \\
\hline O55 & 8.8208754333 & 16.1783520119 & 0.5480845168 \\
\hline C56 & 3.0905676585 & 13.6615264504 & 9.7622750891 \\
\hline $\mathrm{C} 57$ & 9.3469608028 & 17.4201829164 & 0.9846008596 \\
\hline C58 & 2.0918549509 & 14.8040023913 & 9.6493939887 \\
\hline C59 & 8.4364135688 & 18.5478388634 & 0.5549587629 \\
\hline O60 & 2.1751786279 & 15.6006891890 & 10.8150486718 \\
\hline O61 & 7.2526621900 & 18.5165144392 & 1.3168989502 \\
\hline
\end{tabular}




\begin{tabular}{|c|c|c|c|}
\hline C62 & 1.1118869708 & 16.5218134175 & 10.9186392095 \\
\hline C63 & 6.4095620826 & 19.6144951449 & 1.0501464512 \\
\hline C64 & 1.3102365520 & 17.3569127416 & 12.1646436222 \\
\hline C65 & 5.3779673131 & 19.7232259180 & 2.1470895172 \\
\hline O66 & 2.2445832912 & 18.3789085113 & 11.8879125527 \\
\hline O67 & 6.0557026232 & 20.0934790477 & 3.3364255663 \\
\hline C68 & 2.6732476011 & 19.0565485189 & 13.0486461841 \\
\hline C69 & 5.3298801322 & 20.1501521825 & 4.4840750984 \\
\hline $\mathrm{C} 70$ & 3.2933197883 & 20.3778695403 & 12.6557106317 \\
\hline C71 & 6.0946379758 & 20.3901001369 & 5.6722471822 \\
\hline $\mathrm{C} 72$ & 3.9627926420 & 20.0278098234 & 4.5438268227 \\
\hline $\mathrm{O} 73$ & 4.4115655404 & 20.1539682225 & 11.8281619689 \\
\hline C74 & 7.5066630424 & 20.5150645528 & 5.6326482625 \\
\hline $\mathrm{C} 75$ & 5.4069288610 & 20.5038849751 & 6.9050885682 \\
\hline C76 & 3.3035798928 & 20.1113113234 & 5.7940590061 \\
\hline C77 & 5.0743003802 & 21.3571703698 & 11.5132067156 \\
\hline $\mathrm{C} 78$ & 8.1975300128 & 20.7847345535 & 6.7871373406 \\
\hline C79 & 6.1652898233 & 20.7709186379 & 8.0879498184 \\
\hline $\mathrm{C} 80$ & 3.9976216115 & 20.3445678469 & 6.9505232057 \\
\hline C81 & 6.1071703371 & 21.1198842682 & 10.4371182178 \\
\hline $\mathrm{C} 82$ & 7.5327622922 & 20.9264469520 & 8.0271756245 \\
\hline $\mathrm{O} 83$ & 5.4254526485 & 20.8822769459 & 9.2209740781 \\
\hline H84 & 8.1593897962 & 10.8071995387 & 6.2101590186 \\
\hline $\mathrm{H} 85$ & 3.8784511146 & 10.1657728274 & 6.8826106788 \\
\hline H86 & 7.7517012095 & 10.3795980184 & 3.8702316583 \\
\hline H87 & 3.6672758799 & 9.7861460666 & 4.5016129126 \\
\hline H88 & 4.1474798180 & 10.6252792467 & 8.9140839911 \\
\hline
\end{tabular}




\begin{tabular}{llll} 
H89 & 8.4369998415 & 11.1236434903 & 8.2127701097 \\
H90 & 4.5884704035 & 11.0157806377 & 11.2574949427 \\
H91 & 8.6834792835 & 11.4749408399 & 10.5869777506 \\
H92 & 7.4035818042 & 12.0016613214 & 1.8772971662 \\
H93 & 3.1748760307 & 11.6741811911 & 2.5773480652 \\
H94 & 7.1755578901 & 14.4292237462 & 1.5835857875 \\
H95 & 2.9391953054 & 14.1142005236 & 2.2396640348 \\
H96 & 8.8027414170 & 13.6998362649 & 12.2588599768 \\
H97 & 4.5434539998 & 13.2310492338 & 12.4418962193 \\
H98 & 8.5153904687 & 16.1389196483 & 11.9282609964 \\
H99 & 4.2478360792 & 15.6496681005 & 12.0078709243 \\
H100 & 5.7278919577 & 16.3835698863 & 1.2415425725 \\
H101 & 3.9643338904 & 16.2565148514 & 1.4221535609 \\
H102 & 2.9822732488 & 16.4055358268 & 3.5630997499 \\
H103 & 7.1211769101 & 16.6159855762 & 3.0494888162 \\
H104 & 3.1560749245 & 16.8172526094 & 5.9548171264 \\
H105 & 7.4938401109 & 17.0253498132 & 5.4067601140 \\
H106 & 7.9772810034 & 17.7620833509 & 9.7363892028 \\
H107 & 3.8495721894 & 17.6880373170 & 10.3547117863 \\
H108 & 7.7334023520 & 17.4211819983 & 7.3552510782 \\
H109 & 3.4111214103 & 17.3377137576 & 7.9882893503 \\
H110 & 10.1613555031 & 12.8956641764 & 5.3245161630 \\
H111 & 10.1761954368 & 14.5196725985 & 4.6062375177 \\
H112 & 10.8831690729 & 12.5807274867 & 3.0883547826 \\
H113 & 9.2251770074 & 11.9564101018 & 3.2335299224 \\
H114 & 9.9085172656 & 14.2794825841 & 6.9499141189 \\
\hline 5.9470143033 & 13.4291176525 & 4.3853493843
\end{tabular}




\begin{tabular}{|c|c|c|c|}
\hline H116 & 9.1011558335 & 14.6006689174 & 9.2724003093 \\
\hline H117 & 10.5458143317 & 15.3437933101 & 2.3998781046 \\
\hline H118 & 11.2853499707 & 14.0501250686 & 1.4343521342 \\
\hline H119 & 3.5516021456 & 13.2910667359 & 4.9317751471 \\
\hline H120 & 9.3515379382 & 14.3906448229 & -0.2338061940 \\
\hline H121 & 10.6568522634 & 15.5990777806 & -0.2015994319 \\
\hline H122 & 3.1564187201 & 13.3709923355 & 10.8147647036 \\
\hline H123 & 2.7464069618 & 12.7886167205 & 9.1944265583 \\
\hline H124 & 10.3379628060 & 17.5865506796 & 0.5362350353 \\
\hline H125 & 9.4583140828 & 17.4425294155 & 2.0771870020 \\
\hline H126 & 2.3051523127 & 15.4083703032 & 8.7561340804 \\
\hline H127 & 1.0746818172 & 14.3894801810 & 9.5567517671 \\
\hline H128 & 8.9629268071 & 19.5021894525 & 0.7141470648 \\
\hline H129 & 8.2180410872 & 18.4599676968 & -0.5211587626 \\
\hline H130 & 1.0753703658 & 17.1823507893 & 10.0384505860 \\
\hline H131 & 0.1524271356 & 15.9842365310 & 10.9791537830 \\
\hline H132 & 6.9958140385 & 20.5455739014 & 1.0098666847 \\
\hline H133 & 5.9019314553 & 19.4942930037 & 0.0802164349 \\
\hline H134 & 0.3528401172 & 17.7979924738 & 12.4810112453 \\
\hline H135 & 1.6683045776 & 16.7069359507 & 12.9773066856 \\
\hline H136 & 4.6358450299 & 20.4869404862 & 1.8740689946 \\
\hline H137 & 4.8721212441 & 18.7617512600 & 2.2957064621 \\
\hline H138 & 1.8222411024 & 19.2620255321 & 13.7160128243 \\
\hline H139 & 3.3951862248 & 18.4396469183 & 13.6060963905 \\
\hline H140 & 3.5856776293 & 20.9163149389 & 13.5721443943 \\
\hline H141 & 2.5483451755 & 20.9932589743 & 12.1284110890 \\
\hline H142 & 3.3823864116 & 19.8326833595 & 3.6491877191 \\
\hline
\end{tabular}




\begin{tabular}{lrrr} 
H143 & 8.0181088214 & 20.3911435401 & 4.6844033692 \\
H144 & 2.2245032970 & 19.9816285930 & 5.8239309959 \\
H145 & 4.3558742230 & 22.1104568214 & 11.1551542945 \\
H146 & 5.5719517351 & 21.7678865553 & 12.4064787327 \\
H147 & 9.2797677098 & 20.8876372278 & 6.7608936912 \\
H148 & 6.7434576998 & 20.2600389540 & 10.6836688710 \\
H149 & 6.7366203932 & 22.0166611786 & 10.3532969987 \\
H150 & 6.6911424288 & 14.4148638149 & 9.7988222739 \\
H151 & 2.7524779210 & 13.5773189169 & 7.2496602766 \\
C152 & 5.4714034360 & 10.2032387864 & 2.6327097561 \\
H153 & 6.3198672771 & 9.7687589035 & 2.0913188489 \\
H154 & 4.5761147914 & 9.6339909539 & 2.3594575596 \\
C155 & 6.8298905849 & 11.7841914007 & 12.3871488608 \\
H156 & 6.0756728044 & 11.3488162951 & 13.0524689667 \\
H157 & 7.8158849024 & 11.5008314730 & 12.7717936801 \\
C158 & 6.1665225658 & 17.5255114867 & 11.5882242950 \\
H159 & 5.3097052862 & 17.9467862249 & 12.1203743474 \\
H160 & 7.0534720491 & 18.1041947665 & 11.8784244030 \\
H161 & 3.4986646489 & 20.3813773766 & 7.9119345329 \\
H162 & 8.1125009010 & 21.1210847090 & 8.9226687672 \\
2 ${ }^{4+}$ CBPQT circling glycol chain & \\
$-3759.049507-3758.381699$ & -3759.403774 & -3760.071581 \\
N1 & 2.0092917280 & 18.6762784229 & 6.3749132439 \\
C2 & 0.8253405058 & 17.7450592036 & 6.3457503846 \\
C3 & 1.8645435573 & 19.9766045605 & 6.0740711214 \\
C4 & 3.2049215691 & 18.1821810637 & 6.7375922835 \\
\hline & 0.6439111729 & 17.1145812144 & 7.7173346001
\end{tabular}




\begin{tabular}{llll} 
C6 & 2.9320623509 & 20.8562443500 & 6.1796065619 \\
C7 & 4.3100784868 & 19.0030040720 & 6.8332582736 \\
C8 & -0.1032953908 & 17.7489980936 & 8.7133389551 \\
C9 & 1.2710234306 & 15.9025530830 & 7.9974955901 \\
C10 & 4.1763638620 & 20.3724978037 & 6.5847573418 \\
C11 & -0.1591796198 & 17.1940332651 & 9.9886869602 \\
C12 & 1.2519424649 & 15.3700745339 & 9.2862680822 \\
C13 & 5.3300005133 & 21.2781783900 & 6.8832220296 \\
C14 & 0.5542215151 & 16.0312301983 & 10.2930482098 \\
C15 & 5.1099101862 & 22.4775726404 & 7.5705704414 \\
C16 & 6.6424320816 & 20.9015382057 & 6.5967724995 \\
C17 & 0.6089159336 & 15.5403041271 & 11.7344505677 \\
C18 & 6.1930127230 & 23.1942688860 & 8.0388184361 \\
C19 & 7.6885310552 & 21.6679949122 & 7.0870617175 \\
N20 & 1.6345220677 & 16.3582537182 & 12.4599028136 \\
N21 & 7.4476780661 & 22.7597220843 & 7.8278047410 \\
C22 & 1.2810444261 & 17.5640385345 & 12.9370045309 \\
C23 & 2.9085090844 & 15.9470635704 & 12.5079724249 \\
C24 & 8.5647831173 & 23.4406688173 & 8.5586765387 \\
C25 & 2.2248481582 & 18.4425747959 & 13.4309413123 \\
C26 & 3.9007771989 & 16.7761788554 & 13.0085764488 \\
C27 & 8.4523257641 & 23.0620581604 & 10.0325856019 \\
C28 & 3.5696643120 & 18.0597817594 & 13.4500824292 \\
C29 & 8.0585891471 & 24.0002387834 & 10.9808318776 \\
C30 & 8.6915297376 & 21.7394869531 & 10.4292332203 \\
\hline & 4.6348763429 & 19.0445966024 & 13.8238607320 \\
\hline
\end{tabular}




\begin{tabular}{llll} 
C33 & 8.5017213379 & 21.3593605496 & 11.7503937622 \\
C34 & 4.4124641437 & 20.4105555336 & 13.5945046279 \\
C35 & 5.8823506300 & 18.6408255746 & 14.2977998222 \\
C36 & 8.0701197417 & 22.2958297944 & 12.6993436834 \\
C37 & 5.4566315987 & 21.2968420884 & 13.7553956233 \\
C38 & 6.8922852004 & 19.5821907324 & 14.4400330926 \\
C39 & 7.8031621020 & 21.8547540209 & 14.1347142487 \\
N40 & 6.6749505986 & 20.8692105058 & 14.1367001884 \\
O41 & 5.5378482305 & 15.2291443616 & 8.6207415896 \\
C42 & 4.6662816018 & 14.1847883531 & 8.6253091301 \\
C43 & 6.0555341481 & 15.7058179692 & 9.8531901614 \\
C44 & 3.9263353584 & 14.0283751548 & 7.4073929413 \\
C45 & 4.4875477892 & 13.3129485185 & 9.6701095463 \\
C46 & 6.1038530867 & 17.2240096768 & 9.7806927098 \\
C47 & 4.1813440066 & 14.8630888908 & 6.2807532471 \\
C48 & 2.9409386568 & 13.0199813575 & 7.3393981478 \\
C49 & 3.5066789117 & 12.2861737581 & 9.5683525356 \\
O50 & 4.7673951653 & 17.6762140516 & 9.6378282202 \\
C51 & 3.4462938753 & 14.6940537744 & 5.1382475090 \\
C52 & 2.1723568340 & 12.8881876095 & 6.1286399827 \\
C53 & 2.7354529410 & 12.1471132035 & 8.4463798866 \\
C54 & 4.5105957264 & 18.9544389374 & 10.1739880513 \\
\hline & 2.4268541510 & 13.7074828437 & 5.0528755476 \\
\hline & 1.2290984057 & 11.9239044984 & 6.1508536303 \\
\hline
\end{tabular}




\begin{tabular}{llll} 
C60 & -0.4877925086 & 10.5407380534 & 5.2050850030 \\
C61 & 1.3298216389 & 20.6683186548 & 10.8360218213 \\
O62 & -1.6534228499 & 11.2396338550 & 5.5941051629 \\
C63 & 1.0855072348 & 21.8449830687 & 11.7675061643 \\
O64 & 1.1282940137 & 21.4323389628 & 13.1304182419 \\
C65 & -0.0388051881 & 21.6965738625 & 13.9025457381 \\
O66 & -4.0112604625 & 11.9922176186 & 7.0911469823 \\
C67 & -1.0186733392 & 20.5327053364 & 13.8282905335 \\
O68 & -1.4460176364 & 20.4152426185 & 12.4765952692 \\
C69 & -2.1758398633 & 19.3237397810 & 12.1124103783 \\
O70 & -5.2280831854 & 14.7123408520 & 7.2763437933 \\
C71 & -2.4511865473 & 18.2616911643 & 12.9390531933 \\
C72 & -2.6486653290 & 19.3402532009 & 10.7543989097 \\
C73 & -3.1823474445 & 17.1468593818 & 12.4343666177 \\
C74 & -3.3921376897 & 18.2348517637 & 10.2897939609 \\
C75 & -2.3870756467 & 20.4437614567 & 9.8905475915 \\
C76 & -5.1321092148 & 17.1127116737 & 7.2606892463 \\
C77 & -3.6387738994 & 17.1227919690 & 11.1462941137 \\
C78 & -3.8890905219 & 18.2513215514 & 8.9402721926 \\
C79 & -2.8651348817 & 20.4178107916 & 8.6084285217 \\
O80 & -4.6263470044 & 17.1755825971 & 8.5905452144 \\
C81 & -3.6179429174 & 19.3172896382 & 8.1162910115 \\
H82 & -0.0330200776 & 18.3392524242 & 6.0444087915 \\
\hline & 1.0329036287 & 16.9998493200 & 5.5796622314 \\
\hline & -0.3379420877 & 15.6783297750 & 12.2522497498 \\
H83 & 0.9078558513 & 14.4977063244 & 11.8071116476 \\
\hline
\end{tabular}




\begin{tabular}{|c|c|c|c|}
\hline H87 & 8.4564320758 & 24.5111790165 & 8.4016478396 \\
\hline H88 & 7.5067471259 & 22.6898344252 & 14.7654173436 \\
\hline H89 & 8.6637285027 & 21.3543265246 & 14.5718944839 \\
\hline H90 & 5.3845101526 & 15.4362387731 & 10.6672390730 \\
\hline H91 & 7.0460448900 & 15.2889779209 & 10.0363912334 \\
\hline H92 & 6.7214276382 & 17.5559690176 & 8.9420121587 \\
\hline H93 & 6.5433220990 & 17.5988943900 & 10.7105941533 \\
\hline H94 & 4.8559791560 & 19.0124340568 & 11.2091712707 \\
\hline H95 & 5.0151168969 & 19.7418021763 & 9.6033622827 \\
\hline H96 & 2.5195739620 & 18.2274226021 & 10.4970555637 \\
\hline H97 & 2.6539453751 & 19.3359982921 & 9.1237647753 \\
\hline H98 & 0.1433897058 & 12.4302637485 & 4.4528524457 \\
\hline H99 & 1.3613156609 & 11.1397457746 & 4.2521341102 \\
\hline H100 & -0.6778485424 & 9.9730695358 & 4.2886945241 \\
\hline H101 & -0.1581539460 & 9.8496855598 & 5.9861095536 \\
\hline H102 & 1.1512863685 & 20.9849783255 & 9.8026542457 \\
\hline H103 & 0.6385032561 & 19.8573486926 & 11.0819974385 \\
\hline H104 & 1.8645512277 & 22.5913917285 & 11.6064330419 \\
\hline H105 & 0.1174125940 & 22.2858299328 & 11.5323299153 \\
\hline H106 & 0.2763934754 & 21.8153940224 & 14.9381191849 \\
\hline H107 & -0.5174804470 & 22.6191360503 & 13.5695394850 \\
\hline H108 & -0.5085628835 & 19.6220649747 & 14.1494585821 \\
\hline H109 & -1.8758585027 & 20.7089315684 & 14.4813373397 \\
\hline H110 & -4.3029533615 & 17.0657147877 & 6.5536102930 \\
\hline H111 & -5.7438871995 & 17.9945008830 & 7.0555727491 \\
\hline H112 & 0.8853176362 & 20.3041895008 & 5.7540222078 \\
\hline H113 & 3.2493753452 & 17.1315567366 & 6.9888570622 \\
\hline
\end{tabular}




\begin{tabular}{|c|c|c|c|}
\hline H114 & 2.7679909307 & 21.8991957060 & 5.9428896484 \\
\hline H115 & 5.2439842592 & 18.5659566634 & 7.1575334922 \\
\hline H116 & -0.6511776956 & 18.6626637180 & 8.5049161257 \\
\hline H117 & 1.7840608884 & 15.3670243991 & 7.2084813357 \\
\hline H118 & -0.7689851711 & 17.6715016089 & 10.7474069758 \\
\hline H119 & 1.7683639569 & 14.4371119071 & 9.4862029530 \\
\hline H120 & 4.1147126789 & 22.8397507143 & 7.7898297909 \\
\hline H121 & 6.8748153802 & 20.0223174845 & 6.0109593123 \\
\hline H122 & 6.0828753720 & 24.1028892442 & 8.6151167897 \\
\hline H123 & 8.7239192204 & 21.4067386743 & 6.9151250220 \\
\hline H124 & 0.2275469912 & 17.8040257976 & 12.8909633835 \\
\hline H125 & 3.1144371095 & 14.9555340696 & 12.1286825419 \\
\hline H126 & 1.9014611922 & 19.4224563241 & 13.7586068957 \\
\hline H127 & 4.9176081529 & 16.4072363128 & 13.0209265779 \\
\hline H128 & 7.8918077794 & 25.0316883260 & 10.6924236111 \\
\hline H129 & 9.0351164966 & 21.0067519326 & 9.7075454306 \\
\hline H130 & 7.5537453470 & 24.3558378585 & 13.0420376970 \\
\hline H131 & 8.6995824620 & 20.3345224877 & 12.0448767810 \\
\hline H132 & 3.4602461409 & 20.7801311881 & 13.2348808195 \\
\hline H133 & 6.1017500285 & 17.6132979932 & 14.5542566577 \\
\hline H134 & 5.3590014152 & 22.3559677199 & 13.5590997549 \\
\hline H135 & 7.8836132211 & 19.3151913961 & 14.7796289831 \\
\hline H136 & 5.0986979426 & 13.3718297274 & 10.5608913690 \\
\hline H137 & 4.9771454274 & 15.5937125571 & 6.3363377873 \\
\hline H138 & 3.3809999611 & 11.6054806704 & 10.4005000454 \\
\hline H139 & 3.6437927745 & 15.3079542058 & 4.2681121542 \\
\hline H140 & 1.9823316386 & 11.3755014917 & 8.3730500908 \\
\hline
\end{tabular}




$\begin{array}{llll}\text { H141 } & 1.8712121644 & 13.6079312082 & 4.1315528218 \\ \text { H142 } & -2.1457642070 & 18.2613660954 & 13.9773043875 \\ \text { H143 } & -3.3808976843 & 16.3158178317 & 13.0990634542 \\ \text { H144 } & -1.8374066999 & 21.2949437295 & 10.2668883689 \\ \text { H145 } & -4.1916211350 & 16.2781760302 & 10.7598935899 \\ \text { H146 } & -2.6878053734 & 21.2563034338 & 7.9465721143 \\ \text { H147 } & -3.9815027169 & 19.3428228165 & 7.0987203669 \\ \text { C148 } & -6.0090001343 & 15.8785921351 & 7.1238305787 \\ \text { H149 } & -6.4670416339 & 15.9124046599 & 6.1296707662 \\ \text { H150 } & -6.8038832912 & 15.9085588111 & 7.8753513547 \\ \text { C151 } & -5.9501625114 & 13.5332605512 & 6.9624038912 \\ \text { H152 } & -6.9664060270 & 13.5963797686 & 7.3656371305 \\ \text { H153 } & -6.0204316899 & 13.4080425943 & 5.8768707566 \\ \text { C154 } & -4.0364432046 & 11.2488512188 & 5.8875111773 \\ \text { H155 } & -4.0939993868 & 11.9070038888 & 5.0168919331 \\ \text { H156 } & -4.8981964424 & 10.5740957246 & 5.8762530805 \\ \text { C157 } & -2.7749125238 & 10.3983879273 & 5.7900937152 \\ \text { H158 } & -2.8857404985 & 9.7169280557 & 4.9395800758 \\ \text { H159 } & -2.6567184686 & 9.8081391964 & 6.7034946453 \\ \text { C160 } & -5.2786644772 & 12.3311814113 & 7.6212954678 \\ \text { H161 } & -5.9565676303 & 11.4742451636 & 7.5523231586 \\ \text { H162 } & -5.1265745155 & 12.5732404845 & 8.6716271258\end{array}$

$\mathbf{1}^{3+} \mathrm{CBPQT}$ circling glycol chain

$-3759.202278-3758.805587-3759.830844-3760.227536$

$\begin{array}{llll}\mathrm{N} 1 & 1.7015849865 & 18.7087980839 & 6.4405787264 \\ \mathrm{C} 2 & 0.6337060647 & 17.6868320780 & 6.4996903649\end{array}$




\begin{tabular}{llll} 
C3 & 1.4490187242 & 19.9977674244 & 6.7956192820 \\
C4 & 2.9828668941 & 18.3383326389 & 6.1660606308 \\
C5 & 0.6003529666 & 17.0418694896 & 7.8811541906 \\
C6 & 2.4471498357 & 20.9184899556 & 6.9275313956 \\
C7 & 4.0281017948 & 19.2057969287 & 6.2781713705 \\
C8 & -0.3768157558 & 17.3808907999 & 8.8117794225 \\
C9 & 1.5875093036 & 16.1180069517 & 8.2305525767 \\
C10 & 3.8184538133 & 20.5574581986 & 6.7070815952 \\
C11 & -0.3531852814 & 16.8215645176 & 10.0914289881 \\
C12 & 1.6265083085 & 15.5757583410 & 9.5078379803 \\
C13 & 4.8978392821 & 21.4571062837 & 6.9371579875 \\
C14 & 0.6602780593 & 15.9355454164 & 10.4492108638 \\
C15 & 4.7168094756 & 22.7231698111 & 7.5862947523 \\
C16 & 6.2523016193 & 21.1444648671 & 6.5815760254 \\
C17 & 0.7829824239 & 15.4247789521 & 11.8772463956 \\
C18 & 5.7900313203 & 23.4732475549 & 7.9576416019 \\
C19 & 7.2835648655 & 21.9471922329 & 6.9690927596 \\
N20 & 1.8201717288 & 16.2632005474 & 12.5640569586 \\
N21 & 7.0711913163 & 23.0828236147 & 7.6929002240 \\
C22 & 1.5123806204 & 17.5426733984 & 12.8375300033 \\
C23 & 3.0579034405 & 15.7992489995 & 12.7711284723 \\
C24 & 8.1744742973 & 23.7121256041 & 8.4338328618 \\
C25 & 2.4639704182 & 18.4294618241 & 13.2933788010 \\
C26 & 4.0630977414 & 16.6418259856 & 13.2257160935 \\
\hline & 8.1929354842 & 23.1795623110 & 9.8712456114 \\
\hline & 8.7825028382 & 17.9867080520 & 13.4722392512 \\
C28
\end{tabular}




\begin{tabular}{|c|c|c|c|}
\hline $\mathrm{C} 30$ & 7.6777663871 & 21.9178746506 & 10.1668860481 \\
\hline C31 & 4.8714037946 & 18.9601428094 & 13.8178694036 \\
\hline $\mathrm{C} 32$ & 8.6633696952 & 23.4915086389 & 12.2306386098 \\
\hline C33 & 7.6512225930 & 21.4482277454 & 11.4750042629 \\
\hline C34 & 4.6275840211 & 20.3408200143 & 13.7643212660 \\
\hline $\mathrm{C} 35$ & 6.1693113039 & 18.5331869863 & 14.1091172360 \\
\hline $\mathrm{C} 36$ & 8.1259360676 & 22.2362166475 & 12.5226141268 \\
\hline $\mathrm{C} 37$ & 5.6798299163 & 21.2202137062 & 13.9140561915 \\
\hline C38 & 7.1902764869 & 19.4646061145 & 14.2242423499 \\
\hline C39 & 8.0530860600 & 21.7530762097 & 13.9728631642 \\
\hline N40 & 6.9371501154 & 20.7740204327 & 14.0932285128 \\
\hline $\mathrm{O} 41$ & 5.8819602041 & 14.8748365191 & 8.9291449755 \\
\hline $\mathrm{C} 42$ & 4.8329736249 & 14.0085242636 & 8.9453505823 \\
\hline $\mathrm{C} 43$ & 6.3598196909 & 15.4520177070 & 10.1398476691 \\
\hline $\mathrm{C} 44$ & 4.0724758362 & 13.9626121425 & 7.7305929753 \\
\hline $\mathrm{C} 45$ & 4.5325243096 & 13.1720130783 & 9.9923982753 \\
\hline $\mathrm{C} 46$ & 6.2234810528 & 16.9635629702 & 10.0468669213 \\
\hline C47 & 4.4198223068 & 14.7763699569 & 6.6108424898 \\
\hline $\mathrm{C} 48$ & 2.9801281517 & 13.0708713484 & 7.6522114047 \\
\hline $\mathrm{C} 49$ & 3.4209696371 & 12.2909477390 & 9.8936633851 \\
\hline $\mathrm{O} 50$ & 4.8467806150 & 17.2780051932 & 10.0553337887 \\
\hline C51 & 3.6973969838 & 14.6645304646 & 5.4535658812 \\
\hline $\mathrm{C} 52$ & 2.2319463539 & 12.9993357568 & 6.4243691083 \\
\hline C53 & 2.6537316190 & 12.2413710896 & 8.7612890102 \\
\hline C54 & 4.6031835854 & 18.6623493061 & 10.1773278594 \\
\hline C55 & 2.5927755766 & 13.7736682136 & 5.3485815200 \\
\hline O56 & 1.2078826380 & 12.1164318630 & 6.4236889462 \\
\hline
\end{tabular}




\begin{tabular}{|c|c|c|c|}
\hline C57 & 3.0950499479 & 18.8992829367 & 10.0862012500 \\
\hline C58 & 0.6512178586 & 11.7474154103 & 5.1634378592 \\
\hline O59 & 2.8034471533 & 20.0404637965 & 10.8713155573 \\
\hline $\mathrm{C} 60$ & -0.4464430215 & 10.7127238122 & 5.3820061189 \\
\hline C61 & 1.4777842130 & 20.5067822493 & 10.7005401781 \\
\hline O62 & -1.6448278036 & 11.3884427940 & 5.7046230668 \\
\hline C63 & 1.2628855889 & 21.7043916135 & 11.6144436301 \\
\hline O64 & 1.2435485454 & 21.3167802263 & 12.9879247843 \\
\hline C65 & 0.0486695438 & 21.6089255871 & 13.7045279406 \\
\hline O66 & -4.1564776955 & 12.0296217417 & 7.0019102036 \\
\hline C67 & -0.9583043307 & 20.4698988462 & 13.6028433560 \\
\hline O68 & -1.3479128962 & 20.3511845168 & 12.2403220501 \\
\hline C69 & -2.1827631926 & 19.3318386890 & 11.8897777099 \\
\hline O70 & -5.3064064126 & 14.8004508786 & 7.0898925321 \\
\hline C71 & -2.6090472781 & 18.3494797338 & 12.7493447521 \\
\hline $\mathrm{C} 72$ & -2.6065751760 & 19.3417905518 & 10.5166617242 \\
\hline C73 & -3.4598557079 & 17.3127015403 & 12.2692373170 \\
\hline C74 & -3.4568319897 & 18.3069010665 & 10.0697833582 \\
\hline $\mathrm{C} 75$ & -2.1978171449 & 20.3721961543 & 9.6209870219 \\
\hline C76 & -5.1006146493 & 17.1934087216 & 6.9862226833 \\
\hline C77 & -3.8728442249 & 17.2808517548 & 10.9663478926 \\
\hline $\mathrm{C} 78$ & -3.8939812806 & 18.3128330730 & 8.6988844643 \\
\hline C79 & -2.6328476533 & 20.3457206597 & 8.3241109827 \\
\hline O80 & -4.7212028382 & 17.3020961628 & 8.3547348464 \\
\hline C81 & -3.4802695047 & 19.3090417276 & 7.8452740990 \\
\hline H82 & -0.3092675464 & 18.1780049657 & 6.2680698997 \\
\hline H83 & 0.8409160792 & 16.9472910718 & 5.7281064964 \\
\hline
\end{tabular}




\begin{tabular}{|c|c|c|c|}
\hline H84 & -0.1423326493 & 15.5275836679 & 12.4399274338 \\
\hline H85 & 1.1206358381 & 14.3923630700 & 11.9214515969 \\
\hline H86 & 9.1024430199 & 23.4794986091 & 7.9144847160 \\
\hline H87 & 8.0292198862 & 24.7912413537 & 8.4279839223 \\
\hline H88 & 7.8501829663 & 22.5769661079 & 14.6540470549 \\
\hline H89 & 8.9646738392 & 21.2436916013 & 14.2780970916 \\
\hline H90 & 5.7753877399 & 15.1058072958 & 10.9893766334 \\
\hline H91 & 7.4030369718 & 15.1699950596 & 10.2743527602 \\
\hline H92 & 6.6964732028 & 17.3297398256 & 9.1317064158 \\
\hline H93 & 6.7277594012 & 17.4138065700 & 10.9107143884 \\
\hline H94 & 4.9476064911 & 19.0247760731 & 11.1524281470 \\
\hline H95 & 5.1271367649 & 19.2228095902 & 9.3949944149 \\
\hline H96 & 2.5739897817 & 18.0153308470 & 10.4661941238 \\
\hline H97 & 2.7890027827 & 19.0555723066 & 9.0482194154 \\
\hline H98 & 0.2080258428 & 12.6142974819 & 4.6706221527 \\
\hline H99 & 1.4436491497 & 11.3285030245 & 4.5392243467 \\
\hline H100 & -0.5712010588 & 10.1524489158 & 4.4496067542 \\
\hline H101 & -0.1535528351 & 10.0162637708 & 6.1733480025 \\
\hline H102 & 1.3265581468 & 20.8200401713 & 9.6625151514 \\
\hline H103 & 0.7507703349 & 19.7230289779 & 10.9365666478 \\
\hline H104 & 2.0836412842 & 22.4073554227 & 11.4698095118 \\
\hline H105 & 0.3254800300 & 22.1925850153 & 11.3490923144 \\
\hline H106 & 0.3206549541 & 21.7330371218 & 14.7516485628 \\
\hline H107 & -0.3952774038 & 22.5379656836 & 13.3424704085 \\
\hline H108 & -0.4879559097 & 19.5456250530 & 13.9470784157 \\
\hline H109 & -1.8297098478 & 20.6809662436 & 14.2267117981 \\
\hline H110 & -4.2086797613 & 17.0765384420 & 6.3695985795 \\
\hline
\end{tabular}




\begin{tabular}{llll} 
H111 & -5.6473327749 & 18.0894843548 & 6.6823799941 \\
H112 & 0.4096161832 & 20.2453114177 & 6.9634529189 \\
H113 & 3.1224874331 & 17.3111156850 & 5.8618768139 \\
H114 & 2.1622870344 & 21.9286778294 & 7.1801263970 \\
H115 & 5.0141306944 & 18.8284936707 & 6.0506781186 \\
H116 & -1.1671235754 & 18.0728437714 & 8.5479350883 \\
H117 & 2.3372880778 & 15.8186387242 & 7.5085468433 \\
H118 & -1.1352112556 & 17.0770116115 & 10.7975954246 \\
H119 & 2.4139484715 & 14.8774258439 & 9.7660302771 \\
H120 & 3.7367291322 & 23.0960012681 & 7.8407307789 \\
H121 & 6.4957165377 & 20.2717963951 & 5.9934160477 \\
H122 & 5.6825716720 & 24.4008166288 & 8.5040118868 \\
H123 & 8.3144345312 & 21.7137787024 & 6.7375070927 \\
H124 & 0.4899152939 & 17.8371749473 & 12.6443953813 \\
H125 & 3.2334603184 & 14.7537270214 & 12.5584935508 \\
H126 & 2.1498787309 & 19.4544523515 & 13.4398211310 \\
H127 & 5.0448278849 & 16.2135803072 & 13.3640800687 \\
H128 & 9.1045344142 & 24.9412027428 & 10.7114418617 \\
H129 & 7.2735382052 & 21.2897646748 & 9.3835624094 \\
H130 & 9.0359934627 & 24.1221089976 & 13.0299365671 \\
H131 & 7.2389882925 & 20.4628953989 & 11.6620274343 \\
H132 & 3.6477533096 & 20.7517546622 & 13.5606712745 \\
H133 & 6.4325036997 & 17.4931462315 & 14.2288694302 \\
H134 & 5.5574454019 & 22.2920711626 & 13.8543466927 \\
\hline & 8.2190473665 & 19.1738696362 & 14.3881090034 \\
\hline & 5.1519470584 & 13.1466294022 & 10.8793773822 \\
H136 & 5.2577840416 & 15.4547322347 & 6.6928691137
\end{tabular}




\begin{tabular}{llll} 
H138 & 3.1982827693 & 11.6394911805 & 10.7288928438 \\
H139 & 3.9673711574 & 15.2513962382 & 4.5833077799 \\
H140 & 1.8102117571 & 11.5702897627 & 8.6831709192 \\
H141 & 2.0554331119 & 13.7134114832 & 4.4129112078 \\
H142 & -2.3237911500 & 18.3544211989 & 13.7925287690 \\
H143 & -3.7790617744 & 16.5431557834 & 12.9602592578 \\
H144 & -1.5688682401 & 21.1713671320 & 9.9862580089 \\
H145 & -4.5105168417 & 16.4907482129 & 10.5959598608 \\
H146 & -2.3461297690 & 21.1326198894 & 7.6375805998 \\
H147 & -3.8015215562 & 19.3287570619 & 6.8136950088 \\
C148 & -6.0127856286 & 15.9900693810 & 6.8138464419 \\
H149 & -6.3645566284 & 15.9994528652 & 5.7767093709 \\
H150 & -6.8796955601 & 16.0846967666 & 7.4752753570 \\
C151 & -6.0151312336 & 13.6477832487 & 6.6719398287 \\
H152 & -7.0704974654 & 13.7334160371 & 6.9537686725 \\
H153 & -5.9622153041 & 13.5450223979 & 5.5833508233 \\
C154 & -4.0447017401 & 11.4186496538 & 5.7323989430 \\
H155 & -3.9896751576 & 12.1652768070 & 4.9372809323 \\
H156 & -4.9066284377 & 10.7686763445 & 5.5485709744 \\
C157 & -2.7885698906 & 10.5550206080 & 5.6935017278 \\
H158 & -2.8108883351 & 9.9613932355 & 4.7732785432 \\
H159 & -2.7799324311 & 9.8758765960 & 6.5514187437 \\
H160 & -5.4659528472 & 12.4093926565 & 7.3774182366 \\
\hline & -6.1612543490 & 11.5809693029 & 7.2011680012 \\
\hline
\end{tabular}

$\mathbf{1}^{2+} \mathrm{CBPQT}$ circling glycol chain 


\begin{tabular}{llll}
-3759.356822 & -3759.156787 & -3760.186610 & -3760.386645 \\
$\mathrm{~N} 1$ & 1.6878946829 & 18.6923953995 & 6.4122039515 \\
$\mathrm{C} 2$ & 0.6268353433 & 17.6631515228 & 6.4828099122 \\
$\mathrm{C} 3$ & 1.4339154696 & 19.9742443578 & 6.7890154717 \\
$\mathrm{C} 4$ & 2.9664561008 & 18.3347998607 & 6.1148617882 \\
$\mathrm{C} 5$ & 0.6010548389 & 17.0268594234 & 7.8674975614 \\
$\mathrm{C} 6$ & 2.4286934214 & 20.8978831372 & 6.9206722292 \\
$\mathrm{C} 7$ & 4.0064460475 & 19.2087047916 & 6.2226860227 \\
$\mathrm{C} 8$ & -0.3732104939 & 17.3737766958 & 8.7977871018 \\
$\mathrm{C} 9$ & 1.5934069366 & 16.1099111719 & 8.2224330859 \\
$\mathrm{C} 10$ & 3.7995351726 & 20.5487292849 & 6.6858973617 \\
$\mathrm{C} 11$ & -0.3454921200 & 16.8201538511 & 10.0798053817 \\
$\mathrm{C} 12$ & 1.6330919248 & 15.5738018927 & 9.5025890335 \\
$\mathrm{C} 13$ & 4.8777534165 & 21.4448736222 & 6.9369088555 \\
$\mathrm{C} 14$ & 0.6643823103 & 15.9324961409 & 10.4426151800 \\
$\mathrm{C} 15$ & 4.6958805136 & 22.6963216404 & 7.6144490037 \\
$\mathrm{C} 16$ & 6.2345748927 & 21.1415697270 & 6.5807801596 \\
$\mathrm{C} 17$ & 0.7821162131 & 15.4240524432 & 11.8740435087 \\
$\mathrm{C} 18$ & 5.7660777527 & 23.4475351165 & 7.9915128196 \\
$\mathrm{C} 19$ & 7.2628159789 & 21.9453954138 & 6.9748887336 \\
$\mathrm{~N} 20$ & 1.7939804144 & 16.2375844089 & 12.5859837272 \\
$\mathrm{~N} 21$ & 7.0481121101 & 23.0735722787 & 7.7089340077 \\
$\mathrm{C} 22$ & 1.5063021864 & 17.5433908541 & 12.8503268886 \\
$\mathrm{C} 23$ & 3.0544248250 & 15.7854590814 & 12.8032877621 \\
$\mathrm{C} 24$ & 8.1531639152 & 23.7107649426 & 8.4412930005 \\
\hline & 2.4514908942 & 18.4254353390 & 13.2736210776 \\
\hline
\end{tabular}




\begin{tabular}{|c|c|c|c|}
\hline $\mathrm{C} 27$ & 8.1785347621 & 23.1891015258 & 9.8815420733 \\
\hline $\mathrm{C} 28$ & 3.8100823601 & 18.0078093783 & 13.4570385251 \\
\hline $\mathrm{C} 29$ & 8.6900778754 & 23.9726316241 & 10.9181462809 \\
\hline $\mathrm{C} 30$ & 7.6621548601 & 21.9292579788 & 10.1847285621 \\
\hline $\mathrm{C} 31$ & 4.8470300785 & 18.9347066845 & 13.7680080071 \\
\hline $\mathrm{C} 32$ & 8.6615522538 & 23.5117130810 & 12.2354118027 \\
\hline $\mathrm{C} 33$ & 7.6386313873 & 21.4680097885 & 11.4958957379 \\
\hline $\mathrm{C} 34$ & 4.6217612191 & 20.3528624864 & 13.7881018410 \\
\hline $\mathrm{C} 35$ & 6.2028074132 & 18.5338388156 & 14.0129021418 \\
\hline $\mathrm{C} 36$ & 8.1250222476 & 22.2582672162 & 12.5368310252 \\
\hline C37 & 5.6568775568 & 21.2205758422 & 13.9516199433 \\
\hline C38 & 7.1967406212 & 19.4557148246 & 14.1657613337 \\
\hline $\mathrm{C} 39$ & 8.0372452690 & 21.7695732025 & 13.9857894914 \\
\hline N40 & 6.9452853491 & 20.7928641889 & 14.1166142296 \\
\hline O41 & 5.8786837110 & 14.8464739263 & 8.9685837537 \\
\hline $\mathrm{C} 42$ & 4.8328917751 & 13.9771931910 & 8.9679518907 \\
\hline $\mathrm{C} 43$ & 6.2994120187 & 15.4531479016 & 10.1885452794 \\
\hline $\mathrm{C} 44$ & 4.0829754835 & 13.9280117279 & 7.7464917874 \\
\hline $\mathrm{C} 45$ & 4.5243868990 & 13.1409429708 & 10.0126948769 \\
\hline $\mathrm{C} 46$ & 6.2197708758 & 16.9642929169 & 10.0409270107 \\
\hline $\mathrm{C} 47$ & 4.4351706848 & 14.7477969820 & 6.6331066626 \\
\hline $\mathrm{C} 48$ & 2.9925680658 & 13.0359342735 & 7.6592672038 \\
\hline C49 & 3.4158408486 & 12.2578985564 & 9.9048548824 \\
\hline $\mathrm{O} 50$ & 4.8597253176 & 17.3120651686 & 9.9253483326 \\
\hline C51 & 3.7231294723 & 14.6389931604 & 5.4698802150 \\
\hline C52 & 2.2530721849 & 12.9696161645 & 6.4260283124 \\
\hline C53 & 2.6583484674 & 12.2059294182 & 8.7657801738 \\
\hline
\end{tabular}




\begin{tabular}{|c|c|c|c|}
\hline C54 & 4.6168814042 & 18.6870837960 & 10.1433088561 \\
\hline C55 & 2.6198300844 & 13.7471929062 & 5.3545859807 \\
\hline O56 & 1.2283207149 & 12.0868366975 & 6.4177181773 \\
\hline C57 & 3.1084047815 & 18.9159083467 & 10.0665692240 \\
\hline C58 & 0.6663503600 & 11.7350648011 & 5.1563897304 \\
\hline O59 & 2.8272479531 & 20.0966733323 & 10.7940873139 \\
\hline C60 & -0.4429437905 & 10.7118536014 & 5.3675489586 \\
\hline C61 & 1.4769016543 & 20.4998089502 & 10.6954019476 \\
\hline O62 & -1.6363968708 & 11.3933185240 & 5.6979398065 \\
\hline C63 & 1.2610072404 & 21.7146137223 & 11.5905482684 \\
\hline O64 & 1.2645686398 & 21.3658031838 & 12.9730634732 \\
\hline C65 & 0.0712119762 & 21.6223266081 & 13.6973068328 \\
\hline O66 & -4.1543984648 & 12.0360904835 & 6.9993662857 \\
\hline C67 & -0.9118118506 & 20.4610349117 & 13.6108783068 \\
\hline O68 & -1.3390311745 & 20.3571420182 & 12.2587356324 \\
\hline C69 & -2.1732270412 & 19.3389154586 & 11.9119098646 \\
\hline $\mathrm{O} 70$ & -5.2904279440 & 14.8151664104 & 7.1014048059 \\
\hline C71 & -2.5909647570 & 18.3503449765 & 12.7678979458 \\
\hline C72 & -2.6079875474 & 19.3577378647 & 10.5425069909 \\
\hline C73 & -3.4484522826 & 17.3198187347 & 12.2870964408 \\
\hline C74 & -3.4639376384 & 18.3299744039 & 10.0950875688 \\
\hline $\mathrm{C} 75$ & -2.1933198828 & 20.3870937670 & 9.6485652527 \\
\hline C76 & -5.0871474294 & 17.2122262509 & 7.0051037910 \\
\hline C77 & -3.8748919895 & 17.2986240835 & 10.9883017114 \\
\hline C78 & -3.8997510301 & 18.3395937692 & 8.7240406072 \\
\hline C79 & -2.6286216305 & 20.3659125330 & 8.3519400719 \\
\hline O80 & -4.7278067758 & 17.3289567288 & 8.3773518515 \\
\hline
\end{tabular}




\begin{tabular}{llll} 
C81 & -3.4813972029 & 19.3338047290 & 7.8714072598 \\
H82 & -0.3201540045 & 18.1466818987 & 6.2507182366 \\
H83 & 0.8392697948 & 16.9229176902 & 5.7136000320 \\
H84 & -0.1624062503 & 15.5125205087 & 12.4091296764 \\
H85 & 1.1104001956 & 14.3871875510 & 11.9038894902 \\
H86 & 9.0789613628 & 23.4771150897 & 7.9177501936 \\
H87 & 8.0049529236 & 24.7893382075 & 8.4275300390 \\
H88 & 7.8420810825 & 22.6047265191 & 14.6572749747 \\
H89 & 8.9605424198 & 21.2801988920 & 14.2928341344 \\
H90 & 5.6423987414 & 15.1614669012 & 11.0035740047 \\
H91 & 7.3196168966 & 15.1392063513 & 10.4029775909 \\
H92 & 6.7856784970 & 17.2942553377 & 9.1649567171 \\
H93 & 6.6608677737 & 17.4180413152 & 10.9366903197 \\
H94 & 4.9524166554 & 18.9782067646 & 11.1432573507 \\
H95 & 5.1392132870 & 19.2993943420 & 9.3989982549 \\
H96 & 2.6036164574 & 18.0539523445 & 10.5091985623 \\
H97 & 2.7833703685 & 19.0137366872 & 9.0262310315 \\
H98 & 0.2369392696 & 12.6101553577 & 4.6708391408 \\
H99 & 1.4514936206 & 11.3145100676 & 4.5247504987 \\
H100 & -0.5742853302 & 10.1596744938 & 4.4315536473 \\
H101 & -0.1548578460 & 10.0080447941 & 6.1535255910 \\
H102 & 1.2466412161 & 20.7777073918 & 9.6613455313 \\
H103 & 0.8005014663 & 19.6938180028 & 10.9942435682 \\
H104 & 2.0754412079 & 22.4194683570 & 11.4181324888 \\
H105 & 0.3155467105 & 22.1903686508 & 11.3290870506 \\
0.3513898770 & 21.7523206672 & 14.7419363768 \\
\hline
\end{tabular}




\begin{tabular}{llll} 
H108 & -0.4129706606 & 19.5414412111 & 13.9249558334 \\
H109 & -1.7689201491 & 20.6461256958 & 14.2626731889 \\
H110 & -4.1875885313 & 17.1012252668 & 6.4017776543 \\
H111 & -5.6330533115 & 18.1041726173 & 6.6888076539 \\
H112 & 0.3965330657 & 20.2129291264 & 6.9792191502 \\
H113 & 3.1090696165 & 17.3119230651 & 5.7984691579 \\
H114 & 2.1426080761 & 21.9010312431 & 7.1993142324 \\
H115 & 4.9911631806 & 18.8403268235 & 5.9769947418 \\
H116 & -1.1622999586 & 18.0676189859 & 8.5311333638 \\
H117 & 2.3465730412 & 15.8131534019 & 7.5022611178 \\
H118 & -1.1228588401 & 17.0826370369 & 10.7886932521 \\
H119 & 2.4245181220 & 14.8821905295 & 9.7692137646 \\
H120 & 3.7174898754 & 23.0583877565 & 7.8884323479 \\
H121 & 6.4842965825 & 20.2738786860 & 5.9888213780 \\
H122 & 5.6562096190 & 24.3638317681 & 8.5557323871 \\
H123 & 8.2941975756 & 21.7182202301 & 6.7402531612 \\
H124 & 0.4812504325 & 17.8369590261 & 12.6703418676 \\
H125 & 3.2251697976 & 14.7366800743 & 12.6027691224 \\
H126 & 2.1431403152 & 19.4517315211 & 13.4196043462 \\
H127 & 5.0227206435 & 16.1716389445 & 13.4044368129 \\
H128 & 9.0880719430 & 24.9590036365 & 10.7065089330 \\
H129 & 7.2487712092 & 21.3013235716 & 9.4048165384 \\
H130 & 9.0369184996 & 24.1463055262 & 13.0307118567 \\
H131 & 7.2101114787 & 20.4938131261 & 11.7009109451 \\
\hline & 3.6384480536 & 20.7703877369 & 13.6190174760 \\
\hline & 5.51976486886 & 22.2929599550 & 13.9303719670
\end{tabular}




\begin{tabular}{llll} 
H135 & 8.2274493150 & 19.1686749320 & 14.3247537782 \\
H136 & 5.1314162163 & 13.1264045943 & 10.9086385078 \\
H137 & 5.2672147849 & 15.4312878895 & 6.7280186885 \\
H138 & 3.1841591530 & 11.6097416389 & 10.7404582324 \\
H139 & 3.9982557929 & 15.2304060204 & 4.6041489481 \\
H140 & 1.8151775975 & 11.5351635293 & 8.6814998108 \\
H141 & 2.0867892723 & 13.6909792768 & 4.4161769848 \\
H142 & -2.2869168274 & 18.3422771117 & 13.8060028990 \\
H143 & -3.7596568117 & 16.5430770951 & 12.9739633312 \\
H144 & -1.5542943429 & 21.1782157407 & 10.0142701470 \\
H145 & -4.5151747162 & 16.5109688154 & 10.6177382490 \\
H146 & -2.3347399357 & 21.1512144407 & 7.6663961652 \\
H147 & -3.7981382988 & 19.3533309995 & 6.8382906369 \\
C148 & -5.9934836193 & 16.0069357658 & 6.8226413266 \\
H149 & -6.3370084706 & 16.0201378330 & 5.7828647852 \\
H150 & -6.8656677387 & 16.1005177014 & 7.4774769628 \\
C151 & -6.0011920572 & 13.6694063270 & 6.6670006204 \\
H152 & -7.0587266745 & 13.7558750347 & 6.9412343449 \\
H153 & -5.9399176581 & 13.5754481398 & 5.5778968259 \\
C154 & -4.0384751760 & 11.4274089905 & 5.7285342300 \\
H155 & -3.9811708718 & 12.1752921443 & 4.9344190701 \\
H156 & -4.9004371238 & 10.7780616784 & 5.5401625783 \\
H157 & -2.7831009527 & 10.5628788573 & 5.6890667439 \\
H158 & -2.8080085967 & 9.9699036712 & 4.7681972703 \\
\hline & -2.7762081937 & 9.8826489872 & 6.5459040387 \\
\hline
\end{tabular}


$\begin{array}{llll}\mathrm{H} 162 & -5.4390453118 & 12.6228657411 & 8.4365136838\end{array}$

$\mathbf{1}^{1+}$ CBPQT circling glycol chain

$-3759.480590-3759.380247-3760.419180-3760.519522$

$\begin{array}{llll}\mathrm{N} 1 & 1.7031512541 & 18.7236090562 & 6.4272612881 \\ \mathrm{C} 2 & 0.6394053876 & 17.6940585087 & 6.4714423128 \\ \mathrm{C} 3 & 1.4399592690 & 20.0049640992 & 6.7978928881 \\ \mathrm{C} 4 & 2.9903957442 & 18.3602734269 & 6.1827418459 \\ \mathrm{C} 5 & 0.5881583066 & 17.0511173261 & 7.8505509981 \\ \mathrm{C} 6 & 2.4311618150 & 20.9281802921 & 6.9581536548 \\ \mathrm{C} 7 & 4.0273308699 & 19.2341478144 & 6.3162282712 \\ \mathrm{C} 8 & -0.3677598109 & 17.4343757007 & 8.7856437022 \\ \mathrm{C} 9 & 1.5477484576 & 16.0993093430 & 8.2020418262 \\ \mathrm{C} 10 & 3.8061106680 & 20.5804999494 & 6.7502858634 \\ \mathrm{C} 11 & -0.3551199093 & 16.8766976413 & 10.0654971921 \\ \mathrm{C} 12 & 1.5713342424 & 15.5626349993 & 9.4821155455 \\ \mathrm{C} 13 & 4.8778985603 & 21.4851456854 & 6.9969270671 \\ \mathrm{C} 14 & 0.6189990764 & 15.9491320658 & 10.4268424835 \\ \mathrm{C} 15 & 4.6865239611 & 22.7444381632 & 7.6576563061 \\ \mathrm{C} 16 & 6.2384933986 & 21.1818602297 & 6.6528141039 \\ \mathrm{C} 17 & 0.7021294088 & 15.4187508435 & 11.8549191937 \\ \mathrm{C} 18 & 5.7512781062 & 23.5077869449 & 8.0264494454 \\ \mathrm{C} 19 & 7.2603804928 & 21.9960318981 & 7.0393831118 \\ \mathrm{~N} 20 & 1.7029697113 & 16.1520744969 & 12.6337631551 \\ \mathrm{~N} 21 & 7.0374660457 & 23.1377509211 & 7.7515259485 \\ \mathrm{C} 22 & 1.4816475667 & 17.5011968584 & 12.8655646053 \\ \mathrm{C} 23 & 3.0027134589 & 15.7008724262 & 12.7477744363\end{array}$




\begin{tabular}{llll} 
C24 & 8.1338075270 & 23.7632765528 & 8.5046107292 \\
C25 & 2.4528588046 & 18.3684393428 & 13.1948655789 \\
C26 & 4.0289833262 & 16.4992659313 & 13.1008306161 \\
C27 & 8.1681680135 & 23.1928851455 & 9.9263971151 \\
C28 & 3.8592073031 & 17.9472249577 & 13.3060835702 \\
C29 & 8.8226362646 & 23.8790648503 & 10.9546251613 \\
C30 & 7.5253014673 & 21.9933680745 & 10.2249600946 \\
C31 & 4.8805911397 & 18.8142625293 & 13.5567762661 \\
C32 & 8.8149391014 & 23.3760421705 & 12.2555269085 \\
C33 & 7.5196738062 & 21.4919261254 & 11.5230684425 \\
C34 & 4.6857419287 & 20.2680037544 & 13.7165168094 \\
C35 & 6.2989747092 & 18.4213990937 & 13.6600469592 \\
C36 & 8.1558722282 & 22.1787136470 & 12.5540966033 \\
C37 & 5.7063520553 & 21.0944905096 & 13.9963874992 \\
C38 & 7.2713182918 & 19.3153069054 & 13.9275764880 \\
C39 & 8.0876146739 & 21.6432593007 & 13.9889277580 \\
N40 & 7.0228188500 & 20.6610511072 & 14.1566117577 \\
O41 & 5.8275051268 & 14.8997616837 & 9.0528920750 \\
C42 & 4.8003659413 & 14.0101999446 & 9.0432967001 \\
C43 & 6.1348239571 & 15.5827402873 & 10.2698031737 \\
C44 & 4.0789016351 & 13.9174034614 & 7.8090334877 \\
C45 & 4.4793912428 & 13.2011174213 & 10.1053083704 \\
C46 & 6.1735906223 & 17.0779588151 & 9.9941354527 \\
C47 & 4.4351236331 & 14.7265778699 & 6.6894736948 \\
C48 & 3.0064703052 & 13.0044784320 & 7.7203706879 \\
C49 & 3.3932451068 & 12.2916196652 & 9.9948646295 \\
\hline & 4.8636578632 & 17.4793219424 & 9.6687403216
\end{tabular}




\begin{tabular}{llll} 
C51 & 3.7463690177 & 14.5851981033 & 5.5164977983 \\
C52 & 2.2894522309 & 12.9060586395 & 6.4765816900 \\
C53 & 2.6658487681 & 12.1931988684 & 8.8387158126 \\
C54 & 4.5729966628 & 18.8242497211 & 10.0006731127 \\
C55 & 2.6627863282 & 13.6694307500 & 5.3978975455 \\
O56 & 1.2746297197 & 12.0096090043 & 6.4674840717 \\
C57 & 3.0563590961 & 18.9833816223 & 9.9869155924 \\
C58 & 0.7161861804 & 11.6568388071 & 5.2059066377 \\
O59 & 2.7663983500 & 20.1928062013 & 10.6616879270 \\
C60 & -0.3932948417 & 10.6331233094 & 5.4152929973 \\
C61 & 1.3911093354 & 20.5010650634 & 10.6891358221 \\
O62 & -1.5847789886 & 11.3205973641 & 5.7407491761 \\
C63 & 1.1949204883 & 21.7544737615 & 11.5386063220 \\
O64 & 1.2673101509 & 21.4846025921 & 12.9367775635 \\
C65 & 0.0767084745 & 21.6742407919 & 13.6783030367 \\
O66 & -4.1031869679 & 12.0158542636 & 6.9991488571 \\
C67 & -0.8762238204 & 20.4869046407 & 13.5947020152 \\
O68 & -1.3783430231 & 20.4189603880 & 12.2655949717 \\
C69 & -2.1910779565 & 19.3838318931 & 11.9235832695 \\
O70 & -5.2559556164 & 14.8040837406 & 7.1162487302 \\
C71 & -2.5806079421 & 18.3832097367 & 12.7788000246 \\
C72 & -2.6382076660 & 19.3978912156 & 10.5579885513 \\
C73 & -3.4221002568 & 17.3383250933 & 12.3012873274 \\
\hline & -3.4734206252 & 18.3530009738 & 10.1117444187 \\
\hline & -2.2471183351 & 20.4370815860 & 9.6645973772 \\
C75 & -5.0655938715 & 17.2042126300 & 7.0175501842 \\
\hline
\end{tabular}




\begin{tabular}{llll} 
C78 & -3.9104540373 & 18.3554504773 & 8.7412239522 \\
C79 & -2.6814456295 & 20.4076366521 & 8.3681931888 \\
O80 & -4.7186193270 & 17.3278782236 & 8.3917340400 \\
C81 & -3.5139978710 & 19.3587818944 & 7.8890287564 \\
H82 & -0.3015252110 & 18.1817845110 & 6.2238314435 \\
H83 & 0.8668527245 & 16.9591876687 & 5.7009784506 \\
H84 & -0.2659588256 & 15.5217694213 & 12.3474376289 \\
H85 & 0.9800471201 & 14.3650265478 & 11.8572692130 \\
H86 & 9.0629973386 & 23.5603422090 & 7.9739568522 \\
H87 & 7.9714027020 & 24.8400289749 & 8.5270740989 \\
H88 & 7.9160112316 & 22.4744162787 & 14.6752229667 \\
H89 & 9.0331408962 & 21.1728774738 & 14.2634313923 \\
H90 & 5.3462814261 & 15.4099846448 & 10.9989187843 \\
H91 & 7.0933393404 & 15.2258508671 & 10.6485702568 \\
H92 & 6.8729226226 & 17.3050549772 & 9.1823642422 \\
H93 & 6.5177208171 & 17.5791616048 & 10.9056743499 \\
H94 & 4.9135320876 & 19.0506485467 & 11.0148004670 \\
H95 & 5.0441158093 & 19.5143049516 & 9.2912336685 \\
H96 & 2.6157317192 & 18.1311074582 & 10.5064800864 \\
H97 & 2.6770539559 & 19.0085535749 & 8.9606224621 \\
H98 & 0.2828765334 & 12.5329930155 & 4.7209112895 \\
H99 & 1.5030617584 & 11.2355096729 & 4.5760389482 \\
H100 & -0.5237450208 & 10.0793433897 & 4.4800760702 \\
H101 & -0.1095775672 & 9.9295695297 & 6.2033986308 \\
H102 & 1.0326097593 & 20.7010787816 & 9.6725584633 \\
\hline & 0.8099868459 & 19.6728771818 & 11.1016224646 \\
\hline 1.9956906087 & 22.4530979832 & 11.2933058479
\end{tabular}




\begin{tabular}{llll} 
H105 & 0.2351420385 & 22.2135890230 & 11.3000032442 \\
H106 & 0.3705846650 & 21.7918313505 & 14.7210314231 \\
H107 & -0.4409717361 & 22.5813789285 & 13.3548555339 \\
H108 & -0.3373076781 & 19.5710069041 & 13.8427594880 \\
H109 & -1.7004820923 & 20.6263176814 & 14.2985830274 \\
H110 & -4.1599730335 & 17.0994506141 & 6.4194234679 \\
H111 & -5.6190132446 & 18.0902153304 & 6.6957446193 \\
H112 & 0.3983269582 & 20.2428659338 & 6.9631686494 \\
H113 & 3.1440344316 & 17.3317702748 & 5.8908296519 \\
H114 & 2.1387102076 & 21.9287050978 & 7.2392558332 \\
H115 & 5.0204389335 & 18.8581569278 & 6.1219318258 \\
H116 & -1.1293728070 & 18.1609688450 & 8.5254480726 \\
H117 & 2.2868881784 & 15.7753601802 & 7.4792345227 \\
H118 & -1.1165655427 & 17.1683871580 & 10.7795593178 \\
H119 & 2.3376290773 & 14.8437922305 & 9.7468556125 \\
H120 & 3.7052641509 & 23.0997937599 & 7.9307837757 \\
H121 & 6.4953505937 & 20.3027940274 & 6.0806195053 \\
H122 & 5.6341197162 & 24.4274549958 & 8.5837440610 \\
H123 & 8.2946132383 & 21.7664358937 & 6.8200246223 \\
H124 & 0.4502363547 & 17.8101596723 & 12.7534759700 \\
H125 & 3.1388976209 & 14.6410486900 & 12.5718042071 \\
H126 & 2.1728851435 & 19.4032319646 & 13.3389762406 \\
H127 & 4.9971837331 & 16.0364008212 & 13.2339427211 \\
H128 & 9.3206559493 & 24.8201769987 & 10.7462109352 \\
\hline & 6.9967899656 & 21.4418672421 & 9.4568619284 \\
\hline
\end{tabular}




\begin{tabular}{llll} 
H132 & 3.7072949432 & 20.7068491575 & 13.5790985598 \\
H133 & 6.5969170447 & 17.3886203203 & 13.5363210210 \\
H134 & 5.5652616736 & 22.1628628297 & 14.1037208520 \\
H135 & 8.3120082162 & 19.0221015342 & 13.9953233901 \\
H136 & 5.0585080353 & 13.2397826760 & 11.0197624810 \\
H137 & 5.2460916878 & 15.4339913287 & 6.7939660602 \\
H138 & 3.1505881387 & 11.6636326601 & 10.8429965118 \\
H139 & 4.0213484117 & 15.1716941211 & 4.6476871512 \\
H140 & 1.8380775239 & 11.5035947133 & 8.7539502823 \\
H141 & 2.1465010581 & 13.5886124406 & 4.4519877969 \\
H142 & -2.2609235423 & 18.3740504930 & 13.8122138224 \\
H143 & -3.7112830255 & 16.5531125404 & 12.9881945908 \\
H144 & -1.6209119001 & 21.2384193080 & 10.0295763401 \\
H145 & -4.4831923587 & 16.5108948188 & 10.6371709339 \\
H146 & -2.4012189758 & 21.1977535641 & 7.6824590083 \\
H147 & -3.8316946418 & 19.3719530631 & 6.8562291946 \\
C148 & -5.9615872096 & 15.9920979452 & 6.8279107485 \\
H149 & -6.2919082823 & 16.0017980613 & 5.7837886157 \\
H150 & -6.8428938126 & 16.0803971874 & 7.4712475721 \\
C151 & -5.9461983459 & 13.6548677920 & 6.6593051123 \\
H152 & -7.0098204713 & 13.7310646599 & 6.9117672353 \\
H153 & -5.8605990942 & 13.5696877229 & 5.5710150552 \\
H154 & -3.9844671871 & 11.3893266794 & 5.7370205748 \\
\hline & -3.9090197225 & 12.1264838793 & 4.9346085971 \\
\hline & -4.8538345581 & 10.7504231615 & 5.5485399132 \\
H157 & -2.7416125425 & 10.5055350141 & 5.7200957611 \\
\hline
\end{tabular}




$\begin{array}{llll}\text { H159 } & -2.7556452029 & 9.8310078868 & 6.5812402072 \\ \text { C160 } & -5.4152999566 & 12.4051772649 & 7.3592988239 \\ \text { H161 } & -6.1146797383 & 11.5847827627 & 7.1646086391 \\ \text { H162 } & -5.3950821383 & 12.6031671293 & 8.4303061207\end{array}$

$\mathbf{1}^{0} \mathrm{CBPQT}$ circling glycol chain

-3759.603488 $-3759.537672-3760.586654-3760.652470$

$\begin{array}{llll}\text { N1 } & 1.4748766132 & 18.6740910583 & 6.4017412228 \\ \text { C2 } & 0.4502075558 & 17.6354571700 & 6.5425373519 \\ \text { C3 } & 1.2706265324 & 19.9193696944 & 6.9695569238 \\ \text { C4 } & 2.7865359748 & 18.2978792966 & 6.1775021351 \\ \text { C5 } & 0.4688465141 & 17.0154209205 & 7.9332926483 \\ \text { C6 } & 2.2693614235 & 20.7842025015 & 7.2279476190 \\ \text { C7 } & 3.8376680350 & 19.1049289882 & 6.4010552823 \\ \text { C8 } & -0.4721868561 & 17.3826506421 & 8.8879444540 \\ \text { C9 } & 1.4831458909 & 16.1213638567 & 8.2870671902 \\ \text { C10 } & 3.6790132984 & 20.4587533973 & 6.9536556043 \\ \text { C11 } & -0.3990701700 & 16.8704973037 & 10.1840630232 \\ \text { C12 } & 1.5657189027 & 15.6232620986 & 9.5797857937 \\ \text { C13 } & 4.7228464644 & 21.2998637110 & 7.1997034080 \\ \text { C14 } & 0.6231793892 & 15.9954510407 & 10.5423667770 \\ \text { C15 } & 4.5873534852 & 22.6185252756 & 7.8438176039 \\ \text { C16 } & 6.1245686051 & 20.9789367320 & 6.8709816219 \\ \text { C17 } & 0.7698209192 & 15.5061004808 & 11.9797111590 \\ \text { C18 } & 5.6504088267 & 23.3976447310 & 8.1042466957 \\ \text { C19 } & 7.1380458471 & 21.8154438129 & 7.1526512815 \\ \text { N20 } & 1.8185169035 & 16.2369175021 & 12.6958332108\end{array}$




\begin{tabular}{|c|c|c|c|}
\hline N21 & 6.9555765909 & 23.0552338119 & 7.7538552757 \\
\hline $\mathrm{C} 22$ & 1.6189332666 & 17.5808658799 & 12.9678888299 \\
\hline $\mathrm{C} 23$ & 3.1201644748 & 15.7790506802 & 12.7104654275 \\
\hline $\mathrm{C} 24$ & 8.0550232652 & 23.6458034473 & 8.5046388303 \\
\hline $\mathrm{C} 25$ & 2.6094641843 & 18.4383971715 & 13.2741699020 \\
\hline $\mathrm{C} 26$ & 4.1680575863 & 16.5613322452 & 13.0315267317 \\
\hline $\mathrm{C} 27$ & 8.1687702447 & 23.0747968430 & 9.9219194952 \\
\hline $\mathrm{C} 28$ & 4.0144369633 & 17.9951597396 & 13.3279270737 \\
\hline $\mathrm{C} 29$ & 8.9900181914 & 23.6862869270 & 10.8766655181 \\
\hline $\mathrm{C} 30$ & 7.4370173491 & 21.9489740258 & 10.2914756477 \\
\hline C31 & 5.0615195189 & 18.8221480278 & 13.6097557545 \\
\hline C32 & 9.0610687889 & 23.1859167224 & 12.1746223501 \\
\hline C33 & 7.5118302388 & 21.4457351874 & 11.5887523396 \\
\hline C34 & 4.9106883091 & 20.2595908559 & 13.8949790043 \\
\hline C35 & 6.4721457362 & 18.3891158693 & 13.6339070179 \\
\hline C36 & 8.3148014305 & 22.0612793387 & 12.5455512700 \\
\hline $\mathrm{C} 37$ & 5.9609248699 & 21.0436016931 & 14.1824926802 \\
\hline C38 & 7.4766172834 & 19.2392850869 & 13.9124096851 \\
\hline C39 & 8.3410132617 & 21.5386802541 & 13.9849642921 \\
\hline $\mathrm{N} 40$ & 7.2760569766 & 20.5759702032 & 14.2394668406 \\
\hline O41 & 5.9827366492 & 14.9481843997 & 8.9735688940 \\
\hline $\mathrm{C} 42$ & 4.9153393355 & 14.1072556226 & 9.0095865533 \\
\hline C43 & 6.3654833978 & 15.6635917558 & 10.1500724021 \\
\hline $\mathrm{C} 44$ & 4.1438334057 & 14.0490116900 & 7.8037144552 \\
\hline $\mathrm{C} 45$ & 4.6206214850 & 13.2875556943 & 10.0702966448 \\
\hline $\mathrm{C} 46$ & 6.1961058354 & 17.1553175194 & 9.9048677753 \\
\hline $\mathrm{C} 47$ & 4.4632926105 & 14.8790847617 & 6.6893414530 \\
\hline
\end{tabular}




\begin{tabular}{|c|c|c|c|}
\hline $\mathrm{C} 48$ & 3.0708329726 & 13.1344993725 & 7.7294517608 \\
\hline C49 & 3.5217093056 & 12.3918801857 & 9.9829620467 \\
\hline $\mathrm{O} 50$ & 4.8220719677 & 17.4066320867 & 9.7761747173 \\
\hline C51 & 3.7482504675 & 14.7485146496 & 5.5314537014 \\
\hline C52 & 2.3271734429 & 13.0452360370 & 6.5008674096 \\
\hline C53 & 2.7624875342 & 12.3085083349 & 8.8462028070 \\
\hline C54 & 4.4819824017 & 18.7636342368 & 10.0032447410 \\
\hline C55 & 2.6718634199 & 13.8232766574 & 5.4238888512 \\
\hline O56 & 1.3195986307 & 12.1382660568 & 6.4968849582 \\
\hline C57 & 2.9620467665 & 18.8606341416 & 10.0471849475 \\
\hline C58 & 0.7653516220 & 11.7845558442 & 5.2340828062 \\
\hline O59 & 2.6568238967 & 20.1074474492 & 10.6501042841 \\
\hline C60 & -0.3300737949 & 10.7437105706 & 5.4334977860 \\
\hline C61 & 1.2759447390 & 20.3809715291 & 10.7068854401 \\
\hline O62 & -1.5421821832 & 11.4079403366 & 5.7366184163 \\
\hline C63 & 1.0886814986 & 21.7255168672 & 11.4071429335 \\
\hline O64 & 1.1457971742 & 21.6189044555 & 12.8292950302 \\
\hline C65 & -0.0753965666 & 21.8225139082 & 13.5120148761 \\
\hline O66 & -4.0917672542 & 11.9972948185 & 6.9951368319 \\
\hline C67 & -1.0162371891 & 20.6238967369 & 13.4459531684 \\
\hline O68 & -1.5143543966 & 20.5273696952 & 12.1173256740 \\
\hline C69 & -2.3108551982 & 19.4764750765 & 11.7901066988 \\
\hline $\mathrm{O} 70$ & -5.2576902689 & 14.7541308436 & 7.0329119161 \\
\hline C71 & -2.7179190398 & 18.5030640120 & 12.6672438241 \\
\hline $\mathrm{C} 72$ & -2.7234795422 & 19.4456813613 & 10.4147858598 \\
\hline C73 & -3.5452670091 & 17.4414663350 & 12.2036318416 \\
\hline $\mathrm{C} 74$ & -3.5467913206 & 18.3843226123 & 9.9822770956 \\
\hline
\end{tabular}




\begin{tabular}{llll} 
C75 & -2.3133221670 & 20.4576936118 & 9.4995095608 \\
C76 & -5.0568700437 & 17.1489559423 & 6.8814163117 \\
C77 & -3.9495486286 & 17.3714397721 & 10.8995823187 \\
C78 & -3.9540307033 & 18.3469001658 & 8.6031701509 \\
C79 & -2.7151581985 & 20.3865582917 & 8.1947034578 \\
O80 & -4.7580605651 & 17.3110395995 & 8.2628582849 \\
C81 & -3.5361741809 & 19.3225203474 & 7.7297020469 \\
H82 & -0.5220473033 & 18.0867458703 & 6.3434717913 \\
H83 & 0.6417785893 & 16.8762816561 & 5.7836108951 \\
H84 & -0.1710529048 & 15.6356678745 & 12.5176410640 \\
H85 & 1.0328367839 & 14.4482019643 & 11.9992791555 \\
H86 & 8.9801586658 & 23.4661563575 & 7.9540001818 \\
H87 & 7.9019182026 & 24.7249345588 & 8.5575969534 \\
H88 & 8.2296412160 & 22.3769642753 & 14.6750501333 \\
H89 & 9.2965399260 & 21.0562853256 & 14.1965726894 \\
H90 & 5.7226912385 & 15.3904651058 & 10.9837501412 \\
H91 & 7.4030418073 & 15.4182335077 & 10.3754330440 \\
H92 & 6.7426842973 & 17.4606217542 & 9.0051220790 \\
H93 & 6.6114882797 & 17.6921895150 & 10.7664497898 \\
H94 & 4.8625829648 & 19.0874788956 & 10.9764988597 \\
H95 & 4.8840741598 & 19.4048326706 & 9.2124818860 \\
H96 & 2.5788314914 & 18.0347405790 & 10.6487928658 \\
H97 & 2.5408957922 & 18.7999147805 & 9.04204948661 \\
H98 & 0.3218520249 & 12.6556866505 & 4.7511702111 \\
H99 & 1.5586931106 & 11.3786097808 & 4.6023051963 \\
\hline & -0.4360280456 & 10.1834325484 & 4.4990415554 \\
\hline
\end{tabular}




\begin{tabular}{llll} 
H102 & 0.8646028021 & 20.4399064241 & 9.6949559502 \\
H103 & 0.7383078231 & 19.5972216138 & 11.2478378055 \\
H104 & 1.9031471614 & 22.3773823803 & 11.0903006971 \\
H105 & 0.1391320416 & 22.1728026885 & 11.1121597368 \\
H106 & 0.1742235640 & 21.9923632317 & 14.5592245885 \\
H107 & -0.5933781928 & 22.7062059664 & 13.1272132761 \\
H108 & -0.4688749062 & 19.7177376971 & 13.7134535658 \\
H109 & -1.8450105683 & 20.7692674196 & 14.1438333361 \\
H110 & -4.1290062411 & 17.0204160544 & 6.3240908920 \\
H111 & -5.5907591633 & 18.0276808494 & 6.5106872285 \\
H112 & 0.2377201109 & 20.1561818534 & 7.1920997169 \\
H113 & 2.9154836258 & 17.2930215951 & 5.8008801669 \\
H114 & 2.0020255093 & 21.7387827467 & 7.6583757255 \\
H115 & 4.8192592526 & 18.7065644521 & 6.1897776628 \\
H116 & -1.2675555339 & 18.0704250556 & 8.6269891852 \\
H117 & 2.2233048344 & 15.8201862722 & 7.5558323241 \\
H118 & -1.1499717988 & 17.1594520306 & 10.9100571674 \\
H119 & 2.3758796327 & 14.9520560858 & 9.8404430001 \\
H120 & 3.6170220806 & 22.9853557139 & 8.1448688512 \\
H121 & 6.3726497739 & 20.0536422722 & 6.3714133572 \\
H122 & 5.5495817827 & 24.3528596043 & 8.6045816451 \\
H123 & 8.1657158113 & 21.5688230615 & 6.9151768628 \\
H124 & 0.5871169116 & 17.8981749523 & 12.8982770546 \\
H125 & 3.2438305871 & 14.7312181393 & 12.4685215280 \\
\hline & 2.3413678365 & 19.4738151544 & 13.4295880683 \\
\hline
\end{tabular}




\begin{tabular}{llll} 
H129 & 6.7811913555 & 21.4631137535 & 9.5805741806 \\
H130 & 9.6864122724 & 23.6839183998 & 12.9087813440 \\
H131 & 6.9142451242 & 20.5819032725 & 11.8503152545 \\
H132 & 3.9349212963 & 20.7224562716 & 13.8520086809 \\
H133 & 6.7378637843 & 17.3609277053 & 13.4330075222 \\
H134 & 5.8475636014 & 22.1026213983 & 14.3773030222 \\
H135 & 8.5113288645 & 18.9197059988 & 13.9129072586 \\
H136 & 5.2358859612 & 13.2968606556 & 10.9612604333 \\
H137 & 5.2676539261 & 15.5946428166 & 6.7855273013 \\
H138 & 3.3004525977 & 11.7553465738 & 10.8305968350 \\
H139 & 3.9952851890 & 15.3527686775 & 4.6663377230 \\
H140 & 1.9313132678 & 11.6211165498 & 8.7756236202 \\
H141 & 2.1361051839 & 13.7500763971 & 4.4879205288 \\
H142 & -2.4206930578 & 18.5273650600 & 13.7069253862 \\
H143 & -3.8476735281 & 16.6761653444 & 12.9073593822 \\
H144 & -1.6962252431 & 21.2707214548 & 9.8534444352 \\
H145 & -4.5633243091 & 16.5572118150 & 10.5417953725 \\
H146 & -2.4169868933 & 21.1539327674 & 7.4907746369 \\
H147 & -3.8266326578 & 19.3022417605 & 6.6887869054 \\
C148 & -5.9519202781 & 15.9359204797 & 6.6957977666 \\
H149 & -6.2538701092 & 15.9193344338 & 5.6433934425 \\
H150 & -6.8503171625 & 16.0433033233 & 7.3120827991 \\
H151 & -5.9531849241 & 13.5975976159 & 6.6077933666 \\
\hline & -7.0152156045 & 13.6807145125 & 6.8648119143 \\
\hline & -5.8751690781 & 13.4865789470 & 5.5220989441 \\
\hline
\end{tabular}




$\begin{array}{llll}\text { H156 } & -4.7917041856 & 10.7441159353 & 5.5109329403 \\ \text { C157 } & -2.6733497934 & 10.5578694646 & 5.7007783174 \\ \text { H158 } & -2.6688370784 & 9.9700874182 & 4.7766178360 \\ \text { H159 } & -2.6711379166 & 9.8725243685 & 6.5533056781 \\ \text { C160 } & -5.4145023125 & 12.3676422533 & 7.3329088239 \\ \text { H161 } & -6.0978113082 & 11.5331145847 & 7.1460624587 \\ \text { H162 } & -5.4104732632 & 12.5857911284 & 8.3987848879\end{array}$

$2^{4+}$ CBPQT circling HQ

$-3451.877057-3451.211417-3452.161349-3452.826989$

O1 $10.2164558295000 \quad 5.7107605994000 \quad 22.0295659920000$

C2 $\quad 11.4910132629000 \quad 5.226347044500022 .4180424917000$

$\begin{array}{llll}\text { C3 } & 9.9964332581000 & 5.9430998576000 & 20.7044303025000\end{array}$

C4 $\quad 11.5103473349000 \quad 5.118519976900023 .9317042645000$

C5 $\quad 10.9007706158000 \quad 5.6168813503000 \quad 19.6859736033000$

$\begin{array}{lllll}\text { C6 } & 8.7728582584000 & 6.5221192035000 & 20.3723869028000\end{array}$

O7 $\quad 10.6537745121000 \quad 4.0621270083000 \quad 24.3250924894000$

C8 $\quad 10.5627869175000 \quad 5.8537028783000 \quad 18.3564580175000$

C9 $\quad 8.4263556823000 \quad 6.7429674897000 \quad 19.0406009238000$

$\begin{array}{llll}\mathrm{C} 10 & 10.6790078872000 & 3.8246697575000 & 25.7239033736000\end{array}$

$\begin{array}{lllll}\text { C11 } & 9.3234841621000 & 6.3990476525000 & 18.0226363520000\end{array}$

$\begin{array}{llll}\text { C12 } & 10.0791555420000 & 2.4589543880000 & 25.9956420047000\end{array}$

$\begin{array}{lllll}013 & 9.0662580193000 & 6.5308422413000 & 16.6902482560000\end{array}$

$\begin{array}{llll}014 & 8.7050620163000 & 2.4470833651000 & 25.6374177314000\end{array}$

$\begin{array}{lllll}\text { C15 } & 7.8114994012000 & 7.0568041183000 & 16.2932331376000\end{array}$

$\begin{array}{lllll}\text { C16 } & 8.1215411204000 & 1.1897185583000 & 25.9512651494000\end{array}$

$\begin{array}{lllll}\text { C17 } & 7.7805839622000 & 7.0981217077000 & 14.7756197740000\end{array}$ 

$\begin{array}{lllll}\mathrm{C} 18 & 6.6290303665000 & 1.2067763727000 & 25.6774392120000\end{array}$
$\begin{array}{lllll}019 & 7.7707405961000 & 5.7759289370000 & 14.2671126686000\end{array}$
$\begin{array}{llll}\text { O20 } & 6.4000894660000 & 1.2881231876000 & 24.2796325634000\end{array}$
$\begin{array}{lllll}\text { C21 } & 7.6070994369000 & 5.7466338193000 & 12.8563297113000\end{array}$
C22 $5.1804537191000 \quad 0.676736406800023 .8986018125000$
C23 $7.5106880511000 \quad 4.302229025200012 .4002983570000$
$\begin{array}{lllll}\text { C24 } & 5.0003928639000 & 0.8354446402000 & 22.4004174367000\end{array}$
O25 $8.7616123036000 \quad 3.6645451683000 \quad 12.6092059635000$
$\begin{array}{llll}\text { O26 } & 6.1038641749000 & 0.2071221466000 & 21.7733501643000\end{array}$
C27 $8.8875049971000 \quad 2.423129641000011 .9281568391000$
$\begin{array}{lllll}\text { C28 } & 6.2429632313000 & 0.3512786815000 & 20.4257013077000\end{array}$
$\begin{array}{lllll}\mathrm{C} 29 & 7.7540872355000 & 1.4532387130000 & 12.2584326561000\end{array}$
$\begin{array}{lllll}\text { C30 } & 5.2933066233000 & 0.9295543306000 & 19.5938570267000\end{array}$
C31 $7.4430612457000 \quad-0.1301399500000 \quad 19.8804354816000$
$\begin{array}{lllll}\text { O32 } & 7.5570543560000 & 1.4520933695000 & 13.6603725784000\end{array}$
C33 $5.5413206172000 \quad 1.0444763684000 \quad 18.2177937749000$
C34 $7.6795906805000 \quad-0.0308113213000 \quad 18.5224034578000$
$\begin{array}{llllll}\text { C35 } & 6.4792139799000 & 0.6157748578000 & 14.0387260373000\end{array}$
C36 $6.7289507904000 \quad 0.564001223700017 .6796444133000$
C37 $\quad 6.0252893996000 \quad 0.9686771570000 \quad 15.4427393545000$
$\begin{array}{lllll}\text { O38 } & 7.0591886084000 & 0.6318631603000 & 16.3568213096000\end{array}$
H39 $11.6791538362000 \quad 4.241630230200021 .9845888187000$
$\begin{array}{llll}H 40 & 12.2723622040000 & 5.9199979979000 & 22.0971834526000\end{array}$

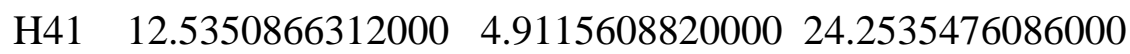
H42 $11.1873950627000 \quad 6.0666838772000 \quad 24.3718622455000$
H43 $11.8600716665000 \quad 5.1700258707000 \quad 19.9123918363000$
H44 $8.0879751828000 \quad 6.776416558900021 .1740961842000$ 


\begin{tabular}{|c|c|c|c|}
\hline 145 & 11.2470597445000 & 5.5961457915000 & 17.5549348542000 \\
\hline 46 & 7.4589107117000 & 7.1707839656000 & 18.8122321942000 \\
\hline 47 & 11.7102064658000 & 3.8231332535000 & 26.0887197887000 \\
\hline$[48$ & 10.1191839919000 & 4.5997027403000 & 26.2564077746000 \\
\hline$[49$ & 10.6223497691000 & 1.7052368512000 & 25.4188595869000 \\
\hline 50 & 10.1877698000000 & 2.2364044078000 & 27.0616026741000 \\
\hline 151 & 6.9968203620000 & 6.4274809953000 & 16.6596711145000 \\
\hline 52 & 7.6832780081000 & 8.0719832263000 & 16.6782942447000 \\
\hline $\mathrm{I} 53$ & 8.5867289564000 & 0.4022483256000 & 25.3529784569000 \\
\hline I54 & 8.2753508512000 & 0.9689011121000 & 27.0120487921000 \\
\hline $\mathrm{I} 55$ & 6.8761702429000 & 5264000 & 14.4636852622000 \\
\hline $\mathrm{I} 56$ & 8.6536081203000 & 7.6434644658000 & 14.4063391543000 \\
\hline H57 & 6.1542927971000 & 2.0474946172000 & 26.1921927292000 \\
\hline $\mathrm{H} 58$ & 6.2135389893000 & 6000 & 68000 \\
\hline I59 & 6.6853159 & 6.2656 & 58000 \\
\hline $\mathrm{H} 60$ & 8.4533231416000 & 6.2364202900000 & 12.3648344405000 \\
\hline H61 & 5.2010838968000 & -0.3869214701000 & 24.1514705163000 \\
\hline $\mathrm{H} 62$ & 4.3322238187000 & 1.1459475843000 & 24.4063685626000 \\
\hline I63 & 6.7291524497000 & 3.7927412322000 & 12.9689484927000 \\
\hline H64 & 7.2544741150000 & 4.2917884853000 & 11.3364830381000 \\
\hline H65 & 4.0610282151000 & 0.3662920575000 & 22.0979178624000 \\
\hline H66 & 4.9718688947000 & 1.8950532679000 & 22.1342890571000 \\
\hline H67 & 8.9080346031000 & 2.5848705968000 & 10.8461759196000 \\
\hline H68 & 9.8406725300000 & 1.9955656523000 & 12.2345050921000 \\
\hline H69 & 8.0273686477000 & 0.4572276537000 & 11.8957198644000 \\
\hline $\mathrm{H} 70$ & 6.8302177572000 & 1.7566968936000 & 11.7564770492000 \\
\hline 11 & 4.3544762659000 & 1.2993745710000 & 19.9848475689000 \\
\hline
\end{tabular}




\begin{tabular}{lllll} 
H72 & 8.1698053417000 & -0.5831197046000 & 20.5433991715000 \\
H73 & 4.7890041293000 & 1.5062099393000 & 17.5925322778000 \\
H74 & 8.5955857698000 & -0.4103977563000 & 18.0861240655000 \\
H75 & 5.6271746739000 & 0.7706100114000 & 13.3691301120000 \\
H76 & 6.7744821432000 & -0.4364522966000 & 13.9939959921000 \\
H77 & 5.1245833945000 & 0.3932969424000 & 15.6693865302000 \\
H78 & 5.8015964143000 & 2.0365494599000 & 15.4992981041000 \\
C79 & 10.4465772222000 & 9.6893645414000 & 20.4088772930000 \\
C80 & 9.0707230979000 & 9.9172013455000 & 20.2921858378000 \\
C81 & 11.0146601930000 & 9.7022961502000 & 21.6845917917000 \\
C82 & 11.2724636568000 & 9.3925250201000 & 19.1975909677000 \\
C83 & 8.3060641952000 & 10.0587637968000 & 21.4325289289000 \\
C84 & 10.1968037970000 & 9.8461294625000 & 22.7919151645000 \\
C85 & 12.4294362238000 & 8.6135443449000 & 19.3012381602000 \\
C86 & 10.8910436017000 & 9.8489330956000 & 17.9330677922000 \\
N87 & 8.8698342844000 & 9.9847181401000 & 22.6501461678000 \\
C88 & 13.1402540894000 & 8.2969557553000 & 18.1586091588000 \\
C89 & 11.6361513933000 & 9.4890495935000 & 16.8244475946000 \\
N90 & 12.7274510919000 & 8.7181138103000 & 16.9519582207000 \\
C91 & 7.5067960776000 & 8.4350082173000 & 23.9189018633000 \\
C92 & 8.3921407460000 & 7.4489592873000 & 24.3604952011000 \\
C93 & 6.2452531293000 & 8.0685314007000 & 23.4481861482000 \\
C94 & 12.8929367263000 & 6.8536873031000 & 15.3824914286000 \\
C95 & 8.0431265594000 & 6.1055646459000 & 24.2784342363000 \\
C96 & 5.8837258167000 & 6.7209372236000 & 23.3911313335000 \\
C98 & 13.5362403404000 & 5.6991680342000 & 15.8362453171000 \\
\hline
\end{tabular}



C99 $\quad 6.7908357948000 \quad 5.735438462900023 .7834856967000$
$\begin{array}{lllll}\text { C100 } & 12.9518253347000 & 4.4490231146000 & 15.6360890054000\end{array}$
$\begin{array}{lllll}\text { C101 } & 11.1006412319000 & 5.4987976888000 & 14.4929302524000\end{array}$
$\begin{array}{llll}\text { C102 } & 6.4707421267000 & 4.2549836202000 & 23.6402623384000\end{array}$
C103 $11.7169127369000 \quad 4.3507247487000 \quad 14.9905433213000$
N104 $7.0095174059000 \quad 3.775445326200022 .3316319662000$
$\begin{array}{llll}\text { C105 } & 10.9759212011000 & 3.0238409720000 & 14.9016666160000\end{array}$
$\begin{array}{llll}\text { C106 } & 8.1941066724000 & 3.1519293032000 & 22.2918480725000\end{array}$
$\begin{array}{lllll}\text { C107 } & 6.3365688596000 & 4.0623892418000 & 21.2027035022000\end{array}$
$\begin{array}{lllll}\text { N108 } & 10.1582870575000 & 2.8765686006000 & 16.1413626001000\end{array}$
$\begin{array}{lllll}\text { C109 } & 8.7759723440000 & 2.8409857975000 & 21.0707567643000\end{array}$
$\begin{array}{lllll}\text { C110 } & 6.8656643886000 & 3.7637780762000 & 19.9657718827000\end{array}$
$\begin{array}{lllll}\text { C111 } & 8.9294723165000 & 3.4087146515000 & 16.1725069897000\end{array}$
$\begin{array}{lllll}\text { C112 } & 10.7091252354000 & 2.3273400620000 & 17.2384455475000\end{array}$
$\begin{array}{lllll}\mathrm{C} 113 & 8.1321232550000 & 3.1718123316000 & 19.8762888512000\end{array}$
$\begin{array}{lllll}\text { C114 } & 8.2291469612000 & 3.4669185176000 & 17.3668400865000\end{array}$
$\begin{array}{lllll}\text { C115 } & 10.0524203538000 & 2.3589389952000 & 18.4487617926000\end{array}$
$\begin{array}{lllll}\text { C116 } & 8.7989191325000 & 2.9777249896000 & 18.5440099369000\end{array}$
$\begin{array}{lllll}H 117 & 8.5647002734000 & 9.9445316398000 & 19.3373057746000\end{array}$
H118 $12.0796110055000 \quad 9.606479182900021 .8464342446000$
H119 $7.2347479478000 \quad 10.204935523000021 .3967802034000$
H120 $10.5869702237000 \quad 9.845207413100023 .8002503116000$
H121 $12.7914089132000 \quad 8.2306205273000 \quad 20.2448729735000$
H122 $10.0387839683000 \quad 10.4976513353000 \quad 17.7836530035000$
$\begin{array}{lllll}H 123 & 14.0407918286000 & 7.6986429558000 & 18.1857338558000\end{array}$
H124 $11.3793486270000 \quad 9.8188851386000 \quad 15.8274823361000$
H125 $9.3596836451000 \quad 7.728504594800024 .7614163742000$ 

H126 $5.5390916560000 \quad 8.825856277500023 .1274091176000$

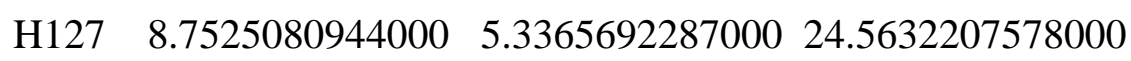
H128 $4.8955972820000 \quad 6.448898509800023 .0373621701000$
H129 $14.4902356816000 \quad 5.769114475100016 .3463535622000$
H130 $11.2102363835000 \quad 7.6331787935000 \quad 14.2894580869000$
H131 $13.4589757111000 \quad 3.5592712554000 \quad 15.9913733673000$
H132 $10.1458822308000 \quad 5.415190532600013 .9866086722000$
H133 $6.9594471156000 \quad 3.662487208700024 .4084375253000$
H134 $5.4001249209000 \quad 4.062493941700023 .6452305606000$
H135 $10.2743287004000 \quad 3.0297713119000 \quad 14.0731259988000$
H136 $11.6447366682000 \quad 2.1685339482000 \quad 14.8420998015000$
$\begin{array}{llll}H 137 & 8.6687187125000 & 2.9444888034000 & 23.2440487276000\end{array}$
$\begin{array}{llll}\mathrm{H} 138 & 5.3771877597000 & 4.5487109973000 & 21.3145408710000\end{array}$
$\begin{array}{llll}H 139 & 9.7428244839000 & 2.3605866393000 & 21.0964228123000\end{array}$
H140 $6.2733229264000 \quad 4.0179661150000 \quad 19.1001970753000$
H141 $8.5452108780000 \quad 3.8199477643000 \quad 15.2456569314000$
H142 $11.6863042822000 \quad 1.8778702658000 \quad 17.1251513300000$
H143 $7.2540515378000 \quad 3.9278033230000 \quad 17.3414760694000$
H144 $10.5480403061000 \quad 1.9045671708000 \quad 19.2937360667000$
$\begin{array}{llll}\text { C145 } & 7.9764269968000 & 9.8827190165000 & 23.8476126855000\end{array}$
H146 $8.5630845338000 \quad 10.173781394700024 .7153013072000$
H147 $7.1545949249000 \quad 10.579816620800023 .7064550478000$
$\begin{array}{lllll}\text { C148 } & 13.4415853875000 & 8.2303535284000 & 15.7272822141000\end{array}$
H149 $13.2566719506000 \quad 8.956679720200014 .9397080148000$
$\begin{array}{llll}\text { H150 } & 14.5030475063000 & 8.2071941661000 & 15.9608739574000\end{array}$

$\mathbf{2}^{3+}$ CBPQT circling BQ 


$\begin{array}{lccc}-3452.029752 & -3451.641784 & -3452.594904 & -3452.982873 \\ \text { O1 } & 6.4680313104 & 6.6758684336 & 16.4019533080 \\ \text { C2 } & 7.8184821200 & 6.8344131817 & 16.2715916426 \\ \text { C3 } & 5.9371256175 & 5.3661753678 & 16.3052979311 \\ \text { C4 } & 8.7380968540 & 5.7799500752 & 16.2373272887 \\ \text { C5 } & 8.2756742995 & 8.1478497088 & 16.2087756962 \\ \text { H6 } & 6.3269277658 & 4.7275140760 & 17.1017353620 \\ \text { H7 } & 6.1866893957 & 4.9268902612 & 15.3360967942 \\ \text { C8 } & 4.4277262693 & 5.4603932412 & 16.4466665762 \\ \text { C9 } & 10.0994377864 & 6.0548167927 & 16.1474375394 \\ \text { H10 } & 8.4105372791 & 4.7494048248 & 16.2703270710 \\ \text { H11 } & 7.5458975788 & 8.9483894447 & 16.2260920292 \\ \text { C12 } & 9.6376362551 & 8.4232215623 & 16.1226809472 \\ \text { O13 } & 4.1195510753 & 5.8081112857 & 17.7858114607 \\ \text { H14 } & 4.0393949720 & 6.2090734265 & 15.7510919417 \\ \text { H15 } & 3.9908450621 & 4.4878338547 & 16.2027891530 \\ \text { C16 } & 10.5564974983 & 7.3693734017 & 16.0918555669 \\ \text { H17 } & 10.8306403398 & 5.2546459054 & 16.1110571974 \\ \text { H18 } & 9.9647145643 & 9.4537690727 & 16.0743527995 \\ \text { C19 } & 2.7265913356 & 5.8511755397 & 18.0473806980 \\ \text { O20 } & 11.9118103274 & 7.5325103134 & 16.0449262582 \\ \text { H21 } & 2.2337092416 & 4.9666057291 & 17.6336556358 \\ \text { H22 } & 2.2770637400 & 6.7417432070 & 17.5985156225 \\ \text { H26 } & 1.4386678003 & 5.8793499037 & 19.7509689598\end{array}$




\begin{tabular}{lccc} 
H27 & 2.9289270349 & 4.9347111698 & 19.9757024082 \\
C28 & 13.9106593897 & 8.7048014244 & 15.5252459677 \\
H29 & 12.2331734297 & 9.5067141206 & 16.5966110068 \\
H30 & 11.9462213843 & 9.2261412624 & 14.8614606227 \\
C31 & 2.9161693386 & 7.0386268336 & 21.5294139242 \\
O32 & 14.5606311242 & 8.3697202735 & 16.7385170298 \\
H33 & 14.0967126102 & 7.9378533302 & 14.7682255754 \\
H34 & 14.2775246348 & 9.6669293607 & 15.1546002082 \\
H35 & 3.1905096402 & 6.0852834233 & 21.9915277368 \\
H36 & 1.8551217936 & 7.2255457285 & 21.7219365976 \\
C37 & 3.7440734731 & 8.1662142933 & 22.1320827510 \\
C38 & 15.9723050292 & 8.4511164236 & 16.6174132491 \\
O39 & 5.1354504277 & 7.8777689780 & 22.0784369254 \\
H40 & 3.5687509007 & 9.0851778856 & 21.5742071598 \\
H41 & 3.4260668290 & 8.3313151561 & 23.1648752666 \\
C42 & 16.6122112866 & 8.0269525515 & 17.9281351212 \\
H43 & 16.2683191208 & 9.4796457628 & 16.3885891055 \\
H44 & 16.3230240360 & 7.7941998342 & 15.8149115703 \\
C45 & 5.5902005142 & 7.2214184578 & 23.2516436982 \\
O46 & 16.3856584858 & 6.6402237024 & 18.1072514657 \\
H47 & 17.6856692836 & 8.2344909169 & 17.8731973204 \\
H48 & 16.1735900449 & 8.5955511760 & 18.7521662098 \\
H49 & 4.9417964000 & 6.3780810486 & 23.5034982498 \\
C50 & 5.5964480345 & 7.9212699819 & 24.0923428015 \\
\hline 6.9908086608 & 6.6770286030 & 23.0401067709 \\
\hline & 17.2194561548 & 6.0231851888 & 19.0725713231 \\
Ho & 7.8669293071 & 7.7734688669 & 22.8319004889
\end{tabular}




\begin{tabular}{|c|c|c|c|}
\hline H54 & 7.2782288809 & 6.1232824023 & 23.9370123053 \\
\hline H55 & 7.0139587348 & 6.0118133558 & 22.1749007518 \\
\hline C56 & 17.1239528080 & 6.6974573179 & 20.4398996007 \\
\hline H57 & 18.2636446955 & 6.0420921047 & 18.7444970222 \\
\hline H58 & 16.8907584096 & 4.9871063910 & 19.1409066889 \\
\hline C59 & 9.2049889439 & 7.5199019586 & 22.7510965662 \\
\hline O60 & 15.7554911577 & 6.8746947043 & 20.7565485515 \\
\hline H61 & 17.6244116641 & 6.0704572844 & 21.1843009960 \\
\hline H62 & 17.6264895008 & 7.6694095279 & 20.4195215311 \\
\hline C63 & 9.7862496807 & 6.2569429943 & 22.8719670333 \\
\hline C64 & 10.0206548739 & 8.6306341391 & 22.5256009260 \\
\hline C65 & 15.5771679748 & 7.6173918451 & 21.9482141730 \\
\hline H66 & 9.1887797689 & 5.3783292226 & 23.0757952453 \\
\hline C67 & 11.1653982366 & 6.1149153459 & 22.7137365959 \\
\hline C68 & 11.3906194574 & 8.4877463472 & 22.3513958186 \\
\hline H69 & 9.5598276031 & 9.6097188812 & 22.4773894556 \\
\hline $\mathrm{C} 70$ & 14.1411853733 & 8.0971667718 & 22.0298245835 \\
\hline $\mathrm{H} 71$ & 16.2221723359 & 8.5017150111 & 21.9419148822 \\
\hline $\mathrm{H} 72$ & 15.8204997343 & 7.0088656985 & 22.8240695071 \\
\hline H73 & 11.6273923355 & 5.1392049097 & 22.8010308055 \\
\hline C74 & 11.9683720149 & 7.2129404100 & 22.4200764114 \\
\hline $\mathrm{H} 75$ & 11.9893786800 & 9.3676876607 & 22.1606680596 \\
\hline $\mathrm{O} 76$ & 13.2957471679 & 6.9702379279 & 22.2079713835 \\
\hline $\mathrm{H} 77$ & 14.0442570338 & 8.7792824020 & 22.8777535500 \\
\hline $\mathrm{H} 78$ & 13.8828225250 & 8.6215675931 & 21.1063321121 \\
\hline N79 & 11.6815217401 & 4.5889475671 & 12.9001628894 \\
\hline C80 & 10.3863319064 & 4.1805510494 & 13.011667306 \\
\hline
\end{tabular}




$\begin{array}{lccc}\text { C81 } & 11.9438675252 & 5.9112523969 & 12.7082231116 \\ \text { C82 } & 12.7941212564 & 3.6587704427 & 13.1860601700 \\ \text { H83 } & 10.2328966089 & 3.1182277322 & 13.1419984042 \\ \text { C84 } & 9.3467479956 & 5.0606149880 & 12.9619484272 \\ \text { C85 } & 10.9514938533 & 6.8414249378 & 12.6623161075 \\ \text { H86 } & 12.9835172640 & 6.1763911230 & 12.5782366904 \\ \text { C87 } & 13.1610214031 & 3.7840336998 & 14.6595312315 \\ \text { H88 } & 13.6298881182 & 3.9312603063 & 12.5446357850 \\ \text { H89 } & 12.4643893262 & 2.6527718971 & 12.9358593620 \\ \text { H90 } & 8.3519421249 & 4.6535051140 & 13.0599607394 \\ \text { C91 } & 9.5776689065 & 6.4688469582 & 12.8239342953 \\ \text { H92 } & 11.2416101295 & 7.8634490503 & 12.4731950776 \\ \text { C93 } & 12.5502511195 & 2.9681180349 & 15.6161640710 \\ \text { C94 } & 14.0278696891 & 4.7931273346 & 15.0755588979 \\ \text { C95 } & 8.5261933210 & 7.4263142643 & 12.8527654520 \\ \text { C96 } & 12.7670542946 & 3.1957003291 & 16.9735086073 \\ \text { H97 } & 11.9026717826 & 2.1564983051 & 15.3039395800 \\ \text { H98 } & 14.5473611003 & 5.3986558065 & 14.3413734514 \\ \text { C99 } & 14.2291699163 & 5.0395287713 & 16.4323722533 \\ \text { C100 } & 7.1452551854 & 7.0462462943 & 12.8682853764 \\ \text { C101 } & 8.7628282597 & 8.8402666023 & 12.8742146264 \\ \text { C102 } & 13.5831305161 & 4.2537722993 & 17.3846236187 \\ \text { H103 } & 12.2906873788 & 2.5511777382 & 17.7038765856 \\ \text { H104 } & 14.8790713191 & 5.8422610365 & 16.7581315180 \\ \text { C107 } & 7.7298890109 & 9.7231497218 & 12.9816747446\end{array}$




\begin{tabular}{lccc} 
H108 & 9.7599396465 & 9.2515712616 & 12.8331703829 \\
C109 & 13.6967951224 & 4.6200270489 & 18.8570363993 \\
N110 & 6.4323054683 & 9.3106853605 & 13.0404218372 \\
H111 & 5.1129963988 & 7.7045241765 & 12.9641280965 \\
H112 & 7.8913734041 & 10.7915055179 & 13.0262672760 \\
N113 & 12.4719662099 & 5.3940904702 & 19.2188127748 \\
H114 & 13.7322487556 & 3.7475675794 & 19.5038728709 \\
H115 & 14.5441811174 & 5.2738221847 & 19.0512643297 \\
C116 & 5.3518943658 & 10.2597667901 & 13.3844270513 \\
C117 & 12.4486586095 & 6.7091087805 & 18.9570537831 \\
C118 & 11.3820251147 & 4.7473218930 & 19.6633046821 \\
H119 & 4.4520788524 & 9.9471645028 & 12.8575603854 \\
H120 & 5.6469205566 & 11.2467674364 & 13.0350432789 \\
C121 & 5.1511338913 & 10.2316727797 & 14.8949227042 \\
C122 & 11.2723445934 & 7.4234631005 & 19.1068948847 \\
H123 & 13.3638902190 & 7.1505942831 & 18.5790938903 \\
H124 & 11.4902056302 & 3.6933211820 & 19.8787230881 \\
C125 & 10.1807425221 & 5.4095613268 & 19.8172984145 \\
C126 & 5.8325238856 & 11.1269736263 & 15.7235896949 \\
C127 & 4.3780738584 & 9.2231408013 & 15.4684778735 \\
C128 & 10.1036191619 & 6.7683970797 & 19.5018596874 \\
H129 & 11.2804780968 & 8.4725435560 & 18.8491067957 \\
H130 & 9.3307258851 & 4.8477123402 & 20.1778556894 \\
H131 & 6.4088631284 & 11.9366594032 & 15.2905442513 \\
\hline & 5.7858826533 & 10.9780028562 & 17.1090082431 \\
H133 & 4.3580306276 & 9.0536641916 & 16.8495876223 \\
\hline
\end{tabular}




$\begin{array}{llll}\text { C135 } & 8.7996637569 & 7.4960751541 & 19.5365612108 \\ \text { H136 } & 6.3173863529 & 11.6824333615 & 17.7385467229 \\ \text { C137 } & 5.0767898745 & 9.9146878314 & 17.6740320180 \\ \text { H138 } & 3.8144372054 & 8.2284851747 & 17.2912615727 \\ \text { C139 } & 8.7625601148 & 8.8888421399 & 19.6791748014 \\ \text { C140 } & 7.5961152369 & 6.8135285508 & 19.3769734727 \\ \text { C141 } & 5.1502564605 & 9.6093237499 & 19.1622990397 \\ \text { C142 } & 7.5508924227 & 9.5372688298 & 19.6170058917 \\ \text { H143 } & 9.6525483593 & 9.4788044572 & 19.8416176720 \\ \text { H144 } & 7.5596551643 & 5.7408465513 & 19.2494939562 \\ \text { C145 } & 6.4006251036 & 7.5176950014 & 19.3169169167 \\ \text { N146 } & 6.4105200111 & 8.8521130922 & 19.4125085626 \\ \text { H147 } & 4.3299611947 & 8.9634285937 & 19.4671712491 \\ \text { H148 } & 5.1797996052 & 10.5078867766 & 19.7745950701 \\ \text { H149 } & 7.4652870149 & 10.6102799828 & 19.7169383397 \\ \text { H150 } & 5.4399961667 & 7.0382845978 & 19.1584633464\end{array}$

$\mathbf{2}^{2+}$ CBPQT circling BQ

\begin{tabular}{|c|c|c|c|}
\hline & $9-3451$ & -3452 & \\
\hline $\mathrm{O} 1$ & 6.5058767812 & 6.6605603914 & 16.3598848667 \\
\hline $\mathrm{C} 2$ & 7.8612998784 & 6.8051223032 & 16.2533488878 \\
\hline C3 & 5.9834368849 & 5.3492113047 & 16.3798734293 \\
\hline $\mathrm{C} 4$ & 8.7715121687 & 5.7428024616 & 16.2451744794 \\
\hline $\mathrm{C} 5$ & 8.3337964622 & 8.1104288693 & 16.1613966149 \\
\hline H6 & 6.3813054818 & 4.7839885313 & 17.2263369349 \\
\hline $\mathrm{H} 7$ & 6.2307354534 & 4.8282549803 & 15.4502669820 \\
\hline $\mathrm{C} 8$ & 4.4720261348 & 5.4361012679 & 16.5170126213 \\
\hline
\end{tabular}




\begin{tabular}{llll} 
C9 & 10.1356854058 & 5.9971373634 & 16.1390992506 \\
H10 & 8.4340486615 & 4.7176242285 & 16.3168886983 \\
H11 & 7.6148793586 & 8.9200648355 & 16.1782911550 \\
C12 & 9.6979419212 & 8.3650559131 & 16.0573822994 \\
O13 & 4.1531630916 & 5.8112271487 & 17.8448944443 \\
H14 & 4.0820727393 & 6.1638409853 & 15.7996645342 \\
H15 & 4.0413774612 & 4.4549155143 & 16.2944978686 \\
C16 & 10.6075978609 & 7.3027295279 & 16.0407031694 \\
H17 & 10.8563450957 & 5.1875829309 & 16.1337031989 \\
H18 & 10.0340382277 & 9.3910745833 & 15.9918624696 \\
C19 & 2.7612652870 & 5.8502858712 & 18.1012717091 \\
O20 & 11.9631180059 & 7.4498727219 & 15.9347735633 \\
H21 & 2.2677391942 & 4.9732120006 & 17.6710909463 \\
H22 & 2.3134119733 & 6.7498864429 & 17.6680418857 \\
C23 & 2.5420832033 & 5.8248054120 & 19.6040758942 \\
C24 & 12.4641792897 & 8.7601603915 & 15.7834571707 \\
O25 & 3.1125312493 & 6.9781744744 & 20.1970359257 \\
H26 & 1.4650656601 & 5.7911310071 & 19.7999050026 \\
H27 & 3.0060492754 & 4.9256526277 & 20.0189239575 \\
C28 & 13.9647418273 & 8.6880316159 & 15.5586521600 \\
H29 & 12.2578827163 & 9.3606489081 & 16.6731114221 \\
H30 & 12.0090150572 & 9.2422878986 & 14.9133495927 \\
C31 & 2.8683554136 & 7.0170754969 & 21.5938534519 \\
H32 & 14.6196234872 & 8.3377176606 & 16.7632377786 \\
\hline & 14.1768307035 & 7.9519365038 & 14.7778046140 \\
H3 3.1433684817 & 6.0591269896 & 22.0463034799
\end{tabular}




\begin{tabular}{lccc} 
H36 & 1.8045789206 & 7.1947661560 & 21.7837342262 \\
C37 & 3.6874389442 & 8.1426085956 & 22.2149996096 \\
C38 & 16.0292805596 & 8.4099166126 & 16.6447073852 \\
O39 & 5.0784189190 & 7.8711559102 & 22.1636858923 \\
H40 & 3.5115661020 & 9.0625929456 & 21.6587190477 \\
H41 & 3.3613417840 & 8.2977615591 & 23.2476589504 \\
C42 & 16.6596531463 & 7.9998278674 & 17.9653520085 \\
H43 & 16.3345990711 & 9.4340537171 & 16.4051845761 \\
H44 & 16.3814676765 & 7.7417162546 & 15.8518585455 \\
C45 & 5.5398048255 & 7.1300641886 & 23.2788465181 \\
O46 & 16.4335805053 & 6.6156290761 & 18.1590273214 \\
H47 & 17.7337153573 & 8.2089638230 & 17.9192455042 \\
H48 & 16.2109620285 & 8.5783858304 & 18.7769759551 \\
H49 & 4.8991909677 & 6.2651843642 & 23.4700570837 \\
H50 & 5.5467949966 & 7.7618957994 & 24.1723925975 \\
C51 & 6.9431441848 & 6.6141849340 & 23.0121883393 \\
C52 & 17.2273293950 & 6.0258883060 & 19.1731545497 \\
O53 & 7.8091062045 & 7.7287407974 & 22.8937733926 \\
H54 & 7.2407367720 & 5.9888852816 & 23.8577022873 \\
H55 & 6.9608018233 & 6.0271063886 & 22.0927984177 \\
C56 & 17.0988431256 & 6.7394925357 & 20.5183408315 \\
H57 & 18.2829557213 & 6.0256640095 & 18.8808535222 \\
H58 & 16.8878454336 & 4.9954062886 & 19.2651975863 \\
C59 & 9.1475149642 & 7.4945501238 & 22.7552298316 \\
\hline & 15.7259709703 & 6.9249724145 & 20.8028023476 \\
\hline
\end{tabular}




\begin{tabular}{llll} 
C63 & 9.7400131723 & 6.2323301285 & 22.8030500792 \\
C64 & 9.9477413208 & 8.6210906073 & 22.5629806239 \\
C65 & 15.5238019385 & 7.6887373133 & 21.9766101884 \\
H66 & 9.1480013052 & 5.3385390238 & 22.9473656259 \\
C67 & 11.1195337358 & 6.1113963005 & 22.6437893113 \\
C68 & 11.3210329607 & 8.4992727577 & 22.3925243352 \\
H69 & 9.4728135140 & 9.5933605583 & 22.5289145527 \\
C70 & 14.0779395791 & 8.1452227660 & 22.0448500255 \\
H71 & 16.1551884130 & 8.5831868796 & 21.9574555508 \\
H72 & 15.7729170040 & 7.1022857067 & 22.8665065307 \\
H73 & 11.5909097267 & 5.1370319603 & 22.6659508691 \\
C74 & 11.9137081936 & 7.2316384089 & 22.4243462354 \\
H75 & 11.9109156862 & 9.3903965116 & 22.2260619676 \\
O76 & 13.2522970192 & 7.0109055149 & 22.2464134238 \\
H77 & 13.9744089282 & 8.8434196545 & 22.8799097491 \\
H78 & 13.8090177662 & 8.6477382259 & 21.1125699419 \\
N79 & 11.6338136848 & 4.6192038507 & 12.8965816717 \\
C80 & 10.3416625432 & 4.2033481469 & 13.0183173860 \\
C81 & 11.8863210172 & 5.9446425425 & 12.7136845915 \\
C82 & 12.7552998302 & 3.6908464697 & 13.1597100447 \\
H83 & 10.1958072661 & 3.1409923022 & 13.1581141142 \\
C84 & 9.2953886831 & 5.0761263997 & 12.9691487599 \\
C85 & 10.8879832876 & 6.8683159474 & 12.6700627337 \\
H86 & 12.9241754920 & 6.2182098551 & 12.5894903947 \\
C87 & 13.1419133932 & 3.7878019752 & 14.6291633476 \\
\hline & 13.5816873466 & 3.9767814423 & 12.5109918955 \\
\hline
\end{tabular}




\begin{tabular}{lccc} 
H90 & 8.3034150207 & 4.6644086502 & 13.0775407292 \\
C91 & 9.5154127803 & 6.4850057456 & 12.8195930865 \\
H92 & 11.1731252121 & 7.8955065653 & 12.4986504731 \\
C93 & 12.5964875927 & 2.9121705595 & 15.5710141834 \\
C94 & 13.9613517679 & 4.8296187906 & 15.0643282881 \\
C95 & 8.4550223283 & 7.4328694438 & 12.8247386480 \\
C96 & 12.8310006379 & 3.1102802130 & 16.9312353550 \\
H97 & 11.9838582586 & 2.0781375549 & 15.2465812703 \\
H98 & 14.4286297278 & 5.48726655566 & 14.3402920146 \\
C99 & 14.1728703084 & 5.0472257407 & 16.4233548830 \\
C100 & 7.0764854172 & 7.0443227803 & 12.8262038249 \\
C101 & 8.6800066284 & 8.8487632425 & 12.8334398744 \\
C102 & 13.5924570607 & 4.1988142295 & 17.3656250787 \\
H103 & 12.4038183875 & 2.4211264583 & 17.6514495868 \\
H104 & 14.7681227323 & 5.8876637070 & 16.7606459947 \\
H105 & 6.7809671363 & 6.0095021413 & 12.7452409534 \\
C106 & 6.0834681748 & 7.9713246975 & 12.9054625664 \\
C107 & 7.6431739182 & 9.7258415979 & 12.9333085450 \\
H108 & 9.6756164952 & 9.2650971324 & 12.8031183708 \\
C109 & 13.6945587832 & 4.5424753093 & 18.8462670337 \\
N110 & 6.3475778710 & 9.3058471771 & 12.9824350907 \\
H111 & 5.0395211951 & 7.6922884717 & 12.8968895395 \\
H112 & 7.7989236996 & 10.7949033265 & 12.9821202428 \\
N113 & 12.5138741547 & 5.3334964749 & 19.2292821980 \\
H114 & 13.7202739768 & 3.6481871208 & 19.4671044272 \\
\hline & 14.5672417770 & 5.1591689451 & 19.0463394335 \\
\hline & 5.2701278749 & 10.2474677118 & 13.3529009532
\end{tabular}




\begin{tabular}{llll} 
C117 & 12.5356217977 & 6.6880498543 & 19.0905233464 \\
C118 & 11.3314448724 & 4.7018714231 & 19.4816599546 \\
H119 & 4.3570744279 & 9.9200882191 & 12.8579228872 \\
H120 & 5.5436486322 & 11.2335235359 & 12.9826105913 \\
C121 & 5.1213456438 & 10.2373156830 & 14.8695236579 \\
C122 & 11.3985362668 & 7.4291531356 & 19.2133785872 \\
H123 & 13.4979135933 & 7.1241312844 & 18.8540753266 \\
H124 & 11.3829300982 & 3.6291558136 & 19.6081155306 \\
C125 & 10.1623008033 & 5.3850262258 & 19.5836167228 \\
C126 & 5.7472124604 & 11.2027998405 & 15.6606794447 \\
C127 & 4.4572936504 & 9.1761955311 & 15.4878362655 \\
C128 & 10.1269231182 & 6.8097397398 & 19.4357780163 \\
H129 & 11.4853802578 & 8.4988088876 & 19.0888925243 \\
H130 & 9.2713271361 & 4.8177134423 & 19.8084622461 \\
H131 & 6.2480499182 & 12.0454260462 & 15.1961086784 \\
C132 & 5.7397614711 & 11.0853088010 & 17.0515169930 \\
C133 & 4.4829552217 & 9.0385983647 & 16.8715689794 \\
H134 & 3.9353333196 & 8.4408032021 & 14.8864699949 \\
C135 & 8.9116324705 & 7.5428851893 & 19.4809365691 \\
H136 & 6.2231485450 & 11.8469196136 & 17.6538294800 \\
C137 & 5.1345715114 & 9.9846762700 & 17.6625320388 \\
H138 & 4.0375985959 & 8.1700023169 & 17.3429785143 \\
C139 & 8.8768674759 & 8.9753221192 & 19.5027021649 \\
C140 & 7.6316522796 & 6.9056875397 & 19.4929767928 \\
\hline & 5.2582882309 & 9.7343694114 & 19.1608411353 \\
\hline H. & 9.7816505198 & 9.5625579153 & 19.5565472580
\end{tabular}




$\begin{array}{llll}\text { H144 } & 7.5402712412 & 5.8302657292 & 19.4849675362 \\ \text { C145 } & 6.4741340318 & 7.6262753740 & 19.4525399960 \\ \text { N146 } & 6.4959452606 & 8.9840689849 & 19.4278869178 \\ \text { H147 } & 4.4292082103 & 9.1219396282 & 19.5098423325 \\ \text { H148 } & 5.2916422440 & 10.6667583730 & 19.7227013458 \\ \text { H149 } & 7.6378084829 & 10.7196210531 & 19.4886118546 \\ \text { H150 } & 5.4972841602 & 7.1602065564 & 19.4222406017\end{array}$

$\mathbf{2}^{1+}$ CBPQT circling BQ

$\begin{array}{llll}-3452.259894-3452.163706 & -3453.131522 & -3453.227710 \\ \text { O1 } & 6.7350690609 & 6.5210899960 & 16.3800252879 \\ \text { C2 } & 8.0881749072 & 6.6917628451 & 16.2536421013 \\ \text { C3 } & 6.2517943347 & 5.2076905939 & 16.5409142332 \\ \text { C4 } & 9.0176791626 & 5.6486059764 & 16.2742976951 \\ \text { C5 } & 8.5329788696 & 8.0000858585 & 16.0936203891 \\ \text { H6 } & 6.6349304238 & 4.7610000373 & 17.4619341868 \\ \text { H7 } & 6.5460539353 & 4.5860265699 & 15.6896052063 \\ \text { C8 } & 4.7334288245 & 5.2634498380 & 16.6008654497 \\ \text { C9 } & 10.3721152646 & 5.9195905199 & 16.1159313872 \\ \text { H10 } & 8.7054693154 & 4.6238064645 & 16.4226135624 \\ \text { H11 } & 7.8022728961 & 8.7983016546 & 16.1105085729 \\ \text { C12 } & 9.8881246554 & 8.2720217917 & 15.9352693090 \\ \text { O13 } & 4.3299108917 & 5.7511583391 & 17.8676228950 \\ \text { H14 } & 4.3700663071 & 5.9120543536 & 15.7985267132 \\ \text { H15 } & 4.3311478198 & 4.2569572305 & 16.4477056248 \\ \text { C16 } & 10.8151171706 & 7.2258732115 & 15.9376244875 \\ \text { H17 } & 11.1067563922 & 5.1254990990 & 16.1534033043\end{array}$




\begin{tabular}{lccc} 
H18 & 10.2063696434 & 9.2993694503 & 15.8213473194 \\
C19 & 2.9304378938 & 5.9380712419 & 17.9701278405 \\
O20 & 12.1623204696 & 7.3893884794 & 15.7608693608 \\
H21 & 2.3993113428 & 5.1375137698 & 17.4456496490 \\
H22 & 2.6418382736 & 6.8973931129 & 17.5304107643 \\
C23 & 2.5238656997 & 5.8829271799 & 19.4334229093 \\
C24 & 12.6667242124 & 8.7030306938 & 15.7042866993 \\
O25 & 3.0798802138 & 6.9768366126 & 20.1361391741 \\
H26 & 1.4301476540 & 5.9099616652 & 19.4945600176 \\
H27 & 2.8761227707 & 4.9404924746 & 19.8630227080 \\
C28 & 14.1769951304 & 8.6346188487 & 15.5325926634 \\
H29 & 12.4269773022 & 9.2496094642 & 16.6197196740 \\
H30 & 12.2483365476 & 9.2388167596 & 14.8456637641 \\
C31 & 2.7455965693 & 6.9403455254 & 21.5118104328 \\
O32 & 14.7822314261 & 8.2232037129 & 16.7433112795 \\
H33 & 14.4156059361 & 7.9360632534 & 14.7247999594 \\
H34 & 14.5417335543 & 9.6291440918 & 15.2535118412 \\
H35 & 3.0061276257 & 5.9645242061 & 21.9337251402 \\
H36 & 1.6687800607 & 7.0939407365 & 21.6451461797 \\
C37 & 3.5008564474 & 8.0495053933 & 22.2363448484 \\
C38 & 16.1947550151 & 8.1964995397 & 16.6666788537 \\
O39 & 4.8996129433 & 7.8343068120 & 22.2233354469 \\
H40 & 3.3143216624 & 8.9945526994 & 21.7270024278 \\
H41 & 3.1279499209 & 8.1282974283 & 23.2624349109 \\
H43 & 16.7610710128 & 7.8838358470 & 18.0434338443 \\
\hline & 16.5730861689 & 9.1739853281 & 16.3480837813 \\
\hline
\end{tabular}




\begin{tabular}{lccc} 
C45 & 5.3558774274 & 6.9941248638 & 23.2663344000 \\
O46 & 16.5031034207 & 6.5278888407 & 18.3582502975 \\
H47 & 17.8407751541 & 8.0697463215 & 18.0276717598 \\
H48 & 16.2899793495 & 8.5427684798 & 18.7769514913 \\
H49 & 4.7331484555 & 6.0999852983 & 23.3572701023 \\
H50 & 5.3315530270 & 7.5318669105 & 24.2198055678 \\
C51 & 6.7759101092 & 6.5403086372 & 22.9714402758 \\
C52 & 17.2093397487 & 6.0539385715 & 19.4916890062 \\
O53 & 7.6101804123 & 7.6830346089 & 22.9661444769 \\
H54 & 7.0797953534 & 5.8453033414 & 23.7594895839 \\
H55 & 6.8199390534 & 6.0465319349 & 22.0006398432 \\
C56 & 16.9936084521 & 6.9069170338 & 20.7416849589 \\
H57 & 18.2841680645 & 6.0138941680 & 19.2831690412 \\
H58 & 16.8487372519 & 5.0431413832 & 19.6745577181 \\
C59 & 8.9482505779 & 7.5072356028 & 22.7408168113 \\
O60 & 15.6068040099 & 7.1027278155 & 20.9180471493 \\
H61 & 17.4332101184 & 6.3845070912 & 21.5986858592 \\
H62 & 17.4993943115 & 7.8728452101 & 20.6402598287 \\
C63 & 9.5911685248 & 6.2685507033 & 22.7373026145 \\
C64 & 9.6946015259 & 8.6654791282 & 22.5379047495 \\
C65 & 15.3173802296 & 7.9679795797 & 21.9972496507 \\
H66 & 9.0351872123 & 5.3485475930 & 22.8575967309 \\
C67 & 10.9711478606 & 6.2089665832 & 22.5654767533 \\
H69 & 11.0709416653 & 8.6047658880 & 22.3516982441 \\
\hline & 1.1767980017 & 9.6146563877 & 22.5038851631 \\
H70 & 13.8481928004 & 8.3529799364 & 21.9723830488 \\
Ho68629714 & 8.8874861836 & 21.9123886519 \\
\hline
\end{tabular}




\begin{tabular}{llll} 
H72 & 15.5566032032 & 7.4876689536 & 22.9518574661 \\
H73 & 11.4824544538 & 5.2557843819 & 22.5470208179 \\
C74 & 11.7195210639 & 7.3672165697 & 22.3826777789 \\
H75 & 11.6192654413 & 9.5202772140 & 22.1765552879 \\
O76 & 13.0745515248 & 7.2063071946 & 22.2691868054 \\
H77 & 13.6913013514 & 9.1260990183 & 22.7301129227 \\
H78 & 13.5793913582 & 8.7394142769 & 20.9878310167 \\
N79 & 11.3723799461 & 4.6387498121 & 12.8608021124 \\
C80 & 10.0811208929 & 4.2354113403 & 13.0112585009 \\
C81 & 11.6334734201 & 5.9599646270 & 12.6617272249 \\
C82 & 12.4908102093 & 3.6924679679 & 13.0742272457 \\
H83 & 9.9310186766 & 3.1771212720 & 13.1746190691 \\
C84 & 9.0403326733 & 5.1151681054 & 12.9663304040 \\
C85 & 10.6418049268 & 6.8919568244 & 12.6298056141 \\
H86 & 12.6705043014 & 6.2247558907 & 12.5163023918 \\
C87 & 12.9494283654 & 3.7610857516 & 14.5245046880 \\
H88 & 13.2906865094 & 3.9738950366 & 12.3909257707 \\
H89 & 12.1362286707 & 2.6976520143 & 12.8125503319 \\
H90 & 8.0467911997 & 4.7173242928 & 13.1075006721 \\
C91 & 9.2699294376 & 6.5190233303 & 12.7962655218 \\
H92 & 10.9294601785 & 7.9162853244 & 12.4465906609 \\
H93 & 12.4900647280 & 2.8424322960 & 15.4703134777 \\
C94 & 13.7540233035 & 4.8217115857 & 14.9423193619 \\
C95 & 8.2167831240 & 7.4746898399 & 12.7981877633 \\
\hline & 12.80539698862 & 3.0103643118 & 16.8196224345 \\
H96 & 11.8813395337 & 2.0004649740 & 15.1583586435 \\
Ho & 14.1405584754 & 5.5241698393 & 14.2140083111
\end{tabular}




\begin{tabular}{lrrr} 
C99 & 14.0416739748 & 5.0069912102 & 16.2909546080 \\
C100 & 6.8356194433 & 7.1005981226 & 12.7395769919 \\
C101 & 8.4571840416 & 8.8866347193 & 12.8714842020 \\
C102 & 13.5596123239 & 4.1086015379 & 17.2437168764 \\
H103 & 12.4468644544 & 2.2879907864 & 17.5449964209 \\
H104 & 14.6069588759 & 5.8733878557 & 16.6157582889 \\
H105 & 6.5364876210 & 6.0741469644 & 12.5904109882 \\
C106 & 5.8500607489 & 8.0341162942 & 12.8380577433 \\
C107 & 7.4286488607 & 9.7666007026 & 13.0025240378 \\
H108 & 9.4588531750 & 9.2884411260 & 12.8911878513 \\
C109 & 13.7803316361 & 4.4040472437 & 18.7242760339 \\
N110 & 6.1263843640 & 9.3591321553 & 13.0038543958 \\
H111 & 4.8026060650 & 7.7712440314 & 12.7826426841 \\
H112 & 7.5915572936 & 10.8288311156 & 13.1254380743 \\
N113 & 12.7102072880 & 5.2249281904 & 19.2782451091 \\
H114 & 13.8329958730 & 3.4725571067 & 19.2904817718 \\
H115 & 14.7093797030 & 4.9576347168 & 18.8499485210 \\
C116 & 5.0798261275 & 10.2768906080 & 13.4960072281 \\
C117 & 12.7519424735 & 6.6019085149 & 19.0755051419 \\
C118 & 11.4549483033 & 4.6494856591 & 19.4329312948 \\
H119 & 4.1259973428 & 9.9316647369 & 13.0999167222 \\
H120 & 5.2873535248 & 11.2726356457 & 13.1083961184 \\
C121 & 5.0973519002 & 10.2568846151 & 15.0215602396 \\
C122 & 11.6558413088 & 7.3777289273 & 19.1196656400 \\
H123 & 13.7412929564 & 7.0025934208 & 18.8935442258 \\
\hline & 11.4569356075 & 3.5728303353 & 19.5551996862 \\
\hline 10.3209901254 & 5.3629227465 & 19.4758471506
\end{tabular}




$\begin{array}{llcc}\text { C126 } & 5.4981344956 & 11.3628894234 & 15.7666155984 \\ \text { C127 } & 4.8015717298 & 9.0614918231 & 15.6856748449 \\ \text { C128 } & 10.3078137322 & 6.8257975680 & 19.3360090140 \\ \text { H129 } & 11.7939482376 & 8.4409954173 & 18.9819583226 \\ \text { H130 } & 9.3988565831 & 4.8263110155 & 19.6483152894 \\ \text { H131 } & 5.7330753603 & 12.2979173168 & 15.2685853916 \\ \text { C132 } & 5.6082233358 & 11.2684189429 & 17.1593713064 \\ \text { C133 } & 4.9353949436 & 8.9590213586 & 17.0624258002 \\ \text { H134 } & 4.4897350748 & 8.1932174352 & 15.1190113636 \\ \text { C135 } & 9.1678968432 & 7.5657582222 & 19.3963960396 \\ \text { H136 } & 5.9204086813 & 12.1366941656 & 17.7303252900 \\ \text { C137 } & 5.3406160829 & 10.0671754694 & 17.8139267129 \\ \text { H138 } & 4.7671886092 & 8.0045177887 & 17.5522749601 \\ \text { C139 } & 9.1361686770 & 9.0317474836 & 19.2955419451 \\ \text { C140 } & 7.8233792957 & 6.9940932618 & 19.5716726093 \\ \text { C141 } & 5.5511593062 & 9.8938208587 & 19.3170271804 \\ \text { C142 } & 7.9941722392 & 9.7314768644 & 19.3908352080 \\ \text { H143 } & 10.0534310898 & 9.5856307860 & 19.1542524586 \\ \text { H144 } & 7.6874201726 & 5.9239634528 & 19.6243373768 \\ \text { C145 } & 6.7185643951 & 7.7550842147 & 19.6589977914 \\ \text { N146 } & 6.7603525018 & 9.1409375291 & 19.6165828169 \\ \text { H147 } & 4.7137272835 & 9.3416303274 & 19.7413425508 \\ \text { H148 } & 5.6118667611 & 10.8714143518 & 19.7989201763 \\ \text { H150 } & 5.7317366609 & 7.3274197117 & 19.7861909917\end{array}$

$\mathbf{2}^{0} \mathrm{CBPQT}$ circling BQ 


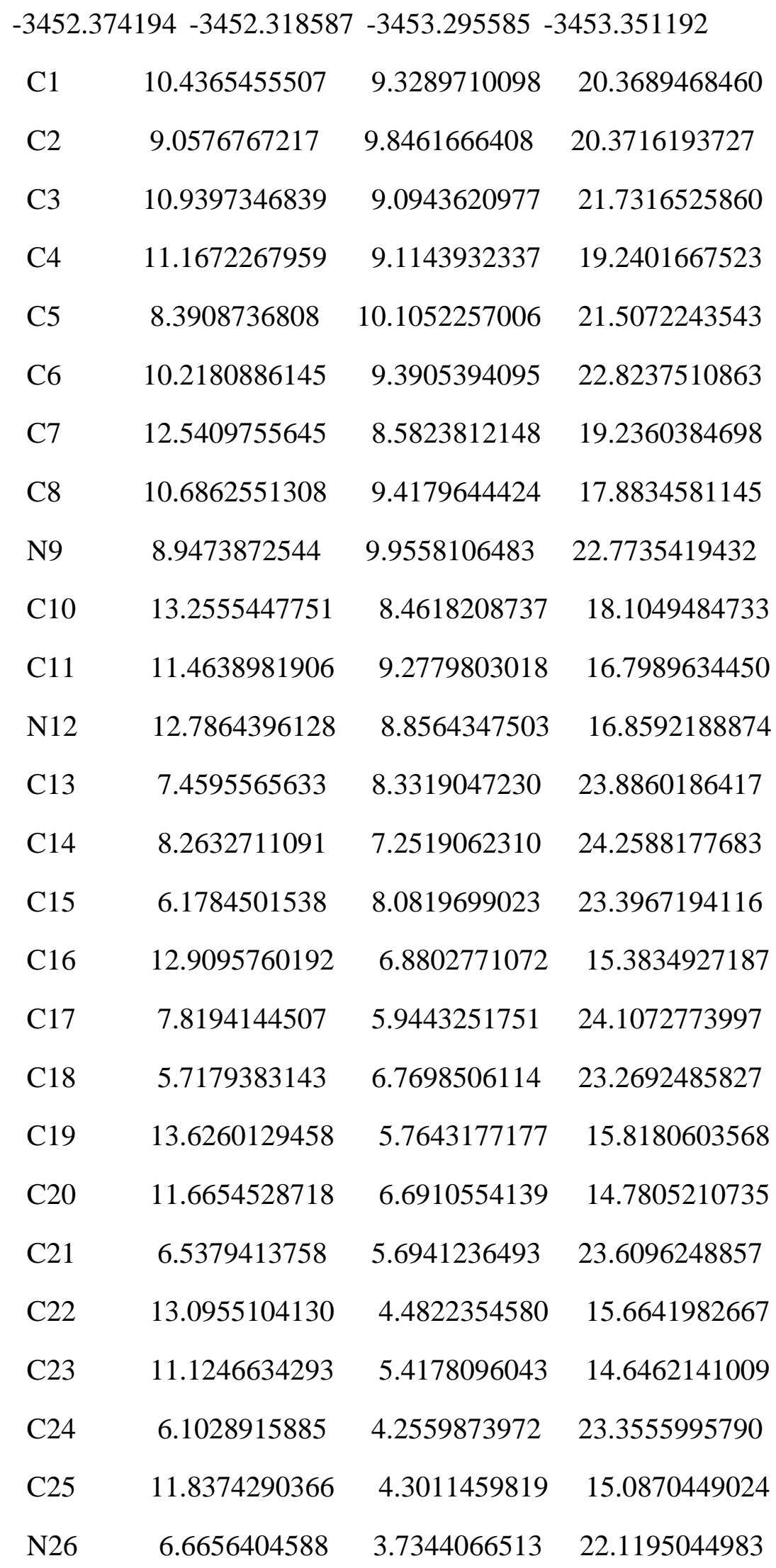




\begin{tabular}{lccc} 
C27 & 11.1949671178 & 2.9226034354 & 14.9772133572 \\
C28 & 7.9352104203 & 3.1706381433 & 22.1283997814 \\
C29 & 6.1634718542 & 4.1621977296 & 20.8991042522 \\
N30 & 10.2793472109 & 2.6303403687 & 16.0740508839 \\
C31 & 8.6309928693 & 2.9551012819 & 21.0024242032 \\
C32 & 6.7991162283 & 3.9620009542 & 19.7336746423 \\
C33 & 9.0191868290 & 3.2267311283 & 16.0606777256 \\
C34 & 10.8105259624 & 2.3161698585 & 17.3191263416 \\
C35 & 8.1200020834 & 3.3160497849 & 19.6716265682 \\
C36 & 8.3051503153 & 3.4381905474 & 17.1765014061 \\
C37 & 10.1417226228 & 2.4876227026 & 18.4694697445 \\
C38 & 8.8023111433 & 3.0936293120 & 18.5158004762 \\
O39 & 10.2111142095 & 5.8229811967 & 22.0738413355 \\
C40 & 11.4690392308 & 5.3282158373 & 22.4577934131 \\
C41 & 9.9801645640 & 6.0042501150 & 20.7362442820 \\
C42 & 11.5163853350 & 5.2846621383 & 23.9767040895 \\
C43 & 10.9053210188 & 5.7018397229 & 19.7338313966 \\
C44 & 8.7400189747 & 6.5262426408 & 20.3905230084 \\
O45 & 10.7058513128 & 4.2202411250 & 24.4388155405 \\
C46 & 10.5868259312 & 5.9253901192 & 18.4003929442 \\
C47 & 8.4204373199 & 6.7475211323 & 19.0554623170 \\
C48 & 10.6293323659 & 4.1535477304 & 25.8481687304 \\
C49 & 9.3449434964 & 6.4451567026 & 18.0538893001 \\
C50 & 10.1274317772 & 2.7771046610 & 26.2522578814 \\
\hline & 9.1230000648 & 6.6350819036 & 16.7153395341 \\
C51 & 8.7743966042 & 2.6074897077 & 25.8706836948 \\
\hline
\end{tabular}




\begin{tabular}{llll} 
C54 & 8.3281399596 & 1.2968305528 & 26.1699191566 \\
C55 & 7.8341937282 & 7.1628277970 & 14.7941711225 \\
C56 & 6.8620472023 & 1.1294114341 & 25.8064681749 \\
O57 & 7.8122179253 & 5.8613232703 & 14.2379342122 \\
O58 & 6.7155072099 & 1.1289538031 & 24.4004709974 \\
C59 & 7.7751154444 & 5.8657442517 & 12.8249413879 \\
C60 & 5.4658096623 & 0.6120250321 & 23.9892848220 \\
C61 & 7.5839086824 & 4.4384436323 & 12.3324829058 \\
C62 & 5.2836507988 & 0.8319228606 & 22.4968937764 \\
O63 & 8.7698841936 & 3.7003393620 & 12.5617789879 \\
O64 & 6.2788580855 & 0.1017711116 & 21.8007130484 \\
C65 & 8.7932345687 & 2.4266498619 & 11.9406710686 \\
C66 & 6.3621012818 & 0.2945436367 & 20.4497444215 \\
C67 & 7.5738396829 & 1.5642925793 & 12.2658866236 \\
C68 & 5.4310462000 & 0.9963366756 & 19.6990386495 \\
C69 & 7.4620976031 & -0.2895253225 & 19.8048026965 \\
O70 & 7.3937908562 & 1.5540149360 & 13.6653064685 \\
C71 & 5.5794411658 & 1.1057225277 & 18.3089291682 \\
C72 & 7.6023210507 & -0.1923739204 & 18.4346116979 \\
H73 & 6.1898792939 & 0.9270645144 & 14.0564672495 \\
C74 & 6.6519109439 & 0.4993456942 & 17.6706036434 \\
C75 & 5.9146874826 & 1.2067789659 & 15.5244155078 \\
O76 & 6.8427763602 & 0.4904616622 & 16.3156248478 \\
H77 & 8.5374398749 & 10.0166501735 & 19.4400936422 \\
\hline & 11.9290279238 & 8.6879661720 & 21.8860161727 \\
H.37188883978 & 10.4724188166 & 21.4978782324 \\
\hline
\end{tabular}




$\begin{array}{lccc}\text { H81 } & 13.0094885810 & 8.2669457726 & 20.1567813410 \\ \text { H82 } & 9.6785599875 & 9.7754833663 & 17.7284908866 \\ \text { H83 } & 14.2633364220 & 8.0656796063 & 18.1077015397 \\ \text { H84 } & 11.1022502010 & 9.5175591213 & 15.8073851135 \\ \text { H85 } & 9.2548093879 & 7.4378800402 & 24.6520959730 \\ \text { H86 } & 5.5376698025 & 8.9092166004 & 23.1093500829 \\ \text { H87 } & 8.4823188951 & 5.1163507097 & 24.3368399968 \\ \text { H88 } & 4.7178824150 & 6.5861162059 & 22.8906431657 \\ \text { H89 } & 14.5997759335 & 5.8940359135 & 16.2788323995 \\ \text { H90 } & 11.1072713749 & 7.5489861002 & 14.4263338194 \\ \text { H91 } & 13.6650691421 & 3.6218379155 & 15.9995070332 \\ \text { H92 } & 10.1334633079 & 5.2865821337 & 14.2263908541 \\ \text { H93 } & 6.4429689536 & 3.6032088105 & 24.1600908873 \\ \text { H94 } & 5.0131809028 & 4.2010329536 & 23.2950127267 \\ \text { H95 } & 10.6189724930 & 2.8751001367 & 14.0546965451 \\ \text { H96 } & 11.9663881252 & 2.1511375528 & 14.9572300630 \\ \text { H97 } & 8.3110751326 & 2.8967182007 & 23.1058200752 \\ \text { H98 } & 5.1960698959 & 4.6478798810 & 20.9501453898 \\ \text { H99 } & 9.6009698748 & 2.4929670451 & 21.1053496928 \\ \text { H100 } & 6.3132026125 & 4.3016491881 & 18.8302543118 \\ \text { H101 } & 8.6475281307 & 3.4907069240 & 15.0784823743 \\ \text { H102 } & 11.8058165969 & 1.8868144331 & 17.2951773579 \\ \text { H103 } & 7.3292850713 & 3.8878238732 & 17.0585395786 \\ \text { H104 } & 10.6290460792 & 2.1731209550 & 19.3815491508 \\ \text { H105 } & 11.6313993962 & 4.3218790821 & 22.0612138778 \\ 12.2681910259 & 5.9876024306 & 22.1028092863 \\ 12.5517838377 & 5.1300689630 & 24.3001873296\end{array}$




\begin{tabular}{llll} 
H108 & 11.1648384667 & 6.2434578957 & 24.3683793505 \\
H109 & 11.8758174795 & 5.2916240652 & 19.9767039146 \\
H110 & 8.0342192993 & 6.7499519260 & 21.1811519908 \\
H111 & 11.2901639973 & 5.6938245651 & 17.6098073351 \\
H112 & 7.4454848498 & 7.1476709193 & 18.8116036547 \\
H113 & 11.6218465827 & 4.2907199470 & 26.2909834145 \\
H114 & 9.9627152724 & 4.9302469539 & 26.2355136085 \\
H115 & 10.7542952961 & 2.0220518669 & 25.7697543871 \\
H116 & 10.2254194783 & 2.6718868669 & 27.3386342045 \\
H117 & 7.0754438191 & 6.3742122340 & 16.6425419063 \\
H118 & 7.6343215496 & 8.0653702999 & 16.7213887434 \\
H119 & 8.9172902964 & 0.5621518969 & 25.6148547412 \\
H120 & 8.4404930574 & 1.1026681141 & 27.2428466261 \\
H121 & 6.9405301920 & 7.7151750566 & 14.4824404765 \\
H122 & 8.7201094896 & 7.7109887585 & 14.4609629561 \\
H123 & 6.2689820605 & 1.9338028155 & 26.2536786358 \\
H124 & 6.5245148880 & 0.1741479975 & 26.2227268959 \\
H125 & 6.9326952490 & 6.4701191098 & 12.4700824855 \\
H126 & 8.7022359861 & 6.2792732981 & 12.4144005533 \\
H127 & 5.4069514326 & -0.4563212551 & 24.2220324597 \\
H128 & 4.6479048949 & 1.1280266415 & 24.5048263585 \\
H129 & 6.7474616538 & 3.9908724217 & 12.8752872250 \\
H130 & 7.3539106735 & 4.4634762339 & 11.2613424242 \\
H131 & 4.2866048824 & 0.4791063696 & 22.2159709525 \\
\hline & 5.3883715010 & 1.8920823841 & 22.2679287823 \\
\hline H132 & 9.8593306705 & 2.5331670530 & 10.8523568288 \\
\hline
\end{tabular}




$\begin{array}{lrrr}\text { H135 } & 7.7503005253 & 0.5545805779 & 11.8790942675 \\ \text { H136 } & 6.6803019739 & 1.9627838308 & 11.7740708772 \\ \text { H137 } & 4.5883686148 & 1.4839412930 & 20.1686355297 \\ \text { H138 } & 8.1947387335 & -0.8136961647 & 20.4049308624 \\ \text { H139 } & 4.8468347769 & 1.6762724467 & 17.7549751450 \\ \text { H140 } & 8.4510163419 & -0.6346549595 & 17.9302271899 \\ \text { H141 } & 5.3517651957 & 1.3313470968 & 13.4779609690 \\ \text { H142 } & 6.2413205393 & -0.1537638213 & 13.8886283879 \\ \text { H143 } & 4.8964710745 & 0.8733269874 & 15.7442543501 \\ \text { H144 } & 6.0054792649 & 2.2771421230 & 15.7171100214 \\ \text { C145 } & 8.0419139540 & 9.7399087078 & 23.9050695794 \\ \text { H146 } & 8.6085028438 & 9.9065093136 & 24.8218473017 \\ \text { H147 } & 7.2461414857 & 10.4831106089 & 23.8512635884 \\ \text { C148 } & 13.4045349100 & 8.2956238166 & 15.6560594213 \\ \text { H149 } & 13.1582617271 & 8.9493623734 & 14.8181639409 \\ \text { H150 } & 14.4860809313 & 8.3052670328 & 15.7937853914\end{array}$

$2^{4+}$ CBPQT circling glycol chain

$\begin{array}{llll}-3451.817540 & -3451.113300 & -3452.064319 & -3452.768559\end{array}$

$\begin{array}{llll}\text { O1 } & 24.9035691394 & 11.3130056851 & 20.5497214771 \\ \text { C2 } & 24.1003002645 & 12.3358544713 & 20.9453595488 \\ \text { C3 } & 25.7988406337 & 11.5247939133 & 19.4648930594 \\ \text { C4 } & 23.1697427558 & 12.0266682682 & 21.9508799007 \\ \text { C5 } & 24.1487575316 & 13.6263742506 & 20.4323728375 \\ \text { H6 } & 26.5068384003 & 12.3186434202 & 19.7092120639 \\ \text { H7 } & 25.2405639072 & 11.7907269835 & 18.5646577334 \\ \text { C8 } & 26.5670695562 & 10.2316879112 & 19.2360816873\end{array}$




\begin{tabular}{llll} 
C9 & 22.3058393341 & 12.9935018862 & 22.4267873681 \\
H10 & 23.1420216306 & 11.0166027175 & 22.3392628859 \\
H11 & 24.8453024234 & 13.8925157574 & 19.6488585908 \\
C12 & 23.2756019636 & 14.6100197024 & 20.9217991597 \\
O13 & 27.4548638452 & 10.0523423940 & 20.3212528670 \\
H14 & 25.8661091302 & 9.3950065430 & 19.1595074384 \\
H15 & 27.1206540076 & 10.3181009433 & 18.2961140614 \\
C16 & 22.3528532735 & 14.2959150693 & 21.9090014425 \\
H17 & 21.5783623992 & 12.7597852246 & 23.1940784145 \\
H18 & 23.3387787299 & 15.6072814153 & 20.5098430951 \\
C19 & 28.2633170602 & 8.8942403815 & 20.2107907438 \\
O20 & 21.4752715129 & 15.1961955070 & 22.4675589075 \\
H21 & 28.8713004404 & 8.9368810001 & 19.3023102588 \\
H22 & 27.6374788077 & 7.9970067684 & 20.1759630155 \\
C23 & 29.1736176524 & 8.8091021889 & 21.4283562797 \\
C24 & 21.4053086356 & 16.4919824111 & 21.8973964711 \\
O25 & 30.1388372460 & 9.8435830030 & 21.3632334040 \\
H26 & 28.5745995083 & 8.9010952283 & 22.3389960835 \\
H27 & 29.6650932551 & 7.8308547852 & 21.4292662917 \\
C28 & 20.2512130361 & 17.2349648503 & 22.5988401465 \\
H29 & 21.2107224646 & 16.4128289653 & 20.8263883564 \\
H30 & 22.3493300014 & 17.0260051656 & 22.0525508443 \\
C31 & 31.0633833037 & 9.8044277636 & 22.4357958717 \\
H32 & 20.4826538294 & 18.6358986042 & 22.5998947392 \\
\hline & 19.3128218429 & 17.0597113990 & 22.0784822867 \\
H3 & 20.1552485513 & 16.8603529215 & 23.6218307370 \\
\hline
\end{tabular}




\begin{tabular}{llll} 
H36 & 30.5502056644 & 9.9672205019 & 23.3882377857 \\
C37 & 32.1167262494 & 10.8848713215 & 22.2329231853 \\
C38 & 21.3790893670 & 19.0333366792 & 23.6215519493 \\
O39 & 31.5083669317 & 12.1576628614 & 22.3663703752 \\
H40 & 32.8979974726 & 10.7555427495 & 22.9885881748 \\
H41 & 32.5654082305 & 10.7780136626 & 21.2405376843 \\
C42 & 21.8224771143 & 20.4636177328 & 23.3412043986 \\
H43 & 22.2681608771 & 18.3979500870 & 23.6299906774 \\
H44 & 20.8950691662 & 18.9683403314 & 24.6007457266 \\
C45 & 32.4230819440 & 13.2310066982 & 22.2553127175 \\
O46 & 22.9562624025 & 20.7172887892 & 24.1392380596 \\
H47 & 22.0694900214 & 20.5440368856 & 22.2773590684 \\
H48 & 21.0149556763 & 21.1643608493 & 23.5718091260 \\
H49 & 32.9394850055 & 13.2005068862 & 21.2909848812 \\
H50 & 33.1681542329 & 13.1909841784 & 23.0559036526 \\
C51 & 31.6455830106 & 14.5357685537 & 22.3446200212 \\
C52 & 23.4064250331 & 22.0608397098 & 24.0454908173 \\
O53 & 31.1540238795 & 14.6573833603 & 23.6726396328 \\
H54 & 32.3038519939 & 15.3743771248 & 22.1075394325 \\
H55 & 30.8147435231 & 14.5091129423 & 21.6386999204 \\
C56 & 24.6412742401 & 22.2174091359 & 24.9172738821 \\
H57 & 22.6274858641 & 22.7457329917 & 24.3905096428 \\
H58 & 23.6576822160 & 22.3006855190 & 23.0070510456 \\
\hline & 24.4044260440 & 21.8944063696 & 25.9324876862
\end{tabular}




$\begin{array}{llll}\text { C63 } & 29.9308066703 & 16.6824645380 & 23.0779023695 \\ \text { C64 } & 29.8316691238 & 15.7147530186 & 25.2875237045 \\ \text { C65 } & 26.2849595944 & 20.5343662085 & 25.2758294383 \\ \text { H66 } & 30.3071583231 & 16.7000183217 & 22.0642477264 \\ \text { C67 } & 29.0393000826 & 17.6861145429 & 23.4836800849 \\ \text { C68 } & 28.9597081872 & 16.7094203489 & 25.6903633922 \\ \text { H69 } & 30.1509861132 & 14.9421158880 & 25.9754176346 \\ \text { C70 } & 27.3775864319 & 19.7766293581 & 24.5197058722 \\ \text { H71 } & 25.5394740094 & 19.8267120425 & 25.6219535030 \\ \text { H72 } & 26.6959324407 & 21.0473203264 & 26.1373200957 \\ \text { H73 } & 28.7491475300 & 18.4457298116 & 22.7702720971 \\ \text { C74 } & 28.5460020313 & 17.6927180452 & 24.7818569144 \\ \text { H75 } & 28.5827337497 & 16.7402289500 & 26.7060740771 \\ \text { O76 } & 27.5938254732 & 18.5709826035 & 25.2449640583 \\ \text { H77 } & 27.0152691175 & 19.5610159259 & 23.5147124657 \\ \text { H78 } & 28.3014163343 & 20.3537840500 & 24.4622132062 \\ \text { N79 } & 23.5659767399 & 19.8198838680 & 19.3086947744 \\ \text { C80 } & 23.2567168664 & 21.1200191332 & 19.4134736918 \\ \text { C81 } & 22.6035217869 & 18.8795487848 & 19.2948792810 \\ \text { C82 } & 24.9887274559 & 19.3642576115 & 19.3353048303 \\ \text { H83 } & 24.0724183823 & 21.8275221596 & 19.3520596823 \\ \text { C84 } & 21.9446271711 & 21.5200609540 & 19.6275127171 \\ \text { C85 } & 21.2869242306 & 19.2141188585 & 19.5214967913 \\ \text { H86 } & 22.9261982908 & 17.8601292700 & 19.1317382983 \\ \text { H88 } & 25.6128586558 & 20.2539128717 & 19.2962773195 \\ \text { H8 } & 25.1629459329 & 18.7598697829 & 18.4476668188\end{array}$




$\begin{array}{llll}\text { H90 } & 21.7413211081 & 22.5788213338 & 19.7166348104 \\ \text { C91 } & 20.9489652131 & 20.5520208073 & 19.7622181954 \\ \text { H92 } & 20.5524652798 & 18.4220310146 & 19.5532852001 \\ \text { C93 } & 25.8390162329 & 17.3118559483 & 20.5530703282 \\ \text { C94 } & 24.7704434041 & 19.0306566545 & 21.8521046122 \\ \text { C95 } & 19.5914779976 & 20.8750582824 & 20.3031336864 \\ \text { C96 } & 25.9958640986 & 16.5432711066 & 21.7082544045 \\ \text { H97 } & 26.1963582018 & 16.9330931853 & 19.6032677245 \\ \text { H98 } & 24.3160436184 & 20.0103317019 & 21.9502559310 \\ \text { C99 } & 24.9147041772 & 18.2575964586 & 23.0013793065 \\ \text { C100 } & 19.4371268083 & 21.9508938226 & 21.1850666883 \\ \text { C101 } & 18.4925842039 & 20.0490360198 & 20.0615637982 \\ \text { C102 } & 25.5197048053 & 16.9983855752 & 22.9365231305 \\ \text { H103 } & 26.4728410738 & 15.5731088664 & 21.6421665569 \\ \text { H104 } & 24.5478888310 & 18.6718468210 & 23.9345123016 \\ \text { H105 } & 20.2344931485 & 22.6516264141 & 21.3898725981 \\ \text { C106 } & 18.2488226074 & 22.1009034547 & 21.8714936438 \\ \text { C107 } & 17.3255423089 & 20.2473806607 & 20.7796261155 \\ \text { H108 } & 18.5170697885 & 19.2428669756 & 19.3412516824 \\ \text { C109 } & 25.6998307903 & 16.1125331233 & 24.1677990381 \\ \text { N110 } & 17.2401503620 & 21.2322113862 & 21.6875569388 \\ \text { H111 } & 18.0896781545 & 22.8874342600 & 22.5968890935 \\ \text { H112 } & 16.4586825731 & 19.6117733999 & 20.6606932342 \\ \text { N113 } & 24.5752195707 & 16.3127311554 & 25.1168667273 \\ \text { H114 } & 25.6988831119 & 15.0610141290 & 23.8907187542 \\ \text { H116 } & 26.6224112186 & 16.3405252801 & 24.6953194676 \\ 16.0734634718 & 21.3010252204 & 22.6037800343\end{array}$




\begin{tabular}{llll} 
C117 & 23.4033536720 & 15.7239319613 & 24.8295851134 \\
C118 & 24.7092824035 & 17.1230561770 & 26.1790640796 \\
H119 & 15.2590692726 & 20.7501695548 & 22.1376323732 \\
H120 & 15.7903919932 & 22.3470589145 & 22.7007303625 \\
C121 & 16.4441050868 & 20.6991930625 & 23.9610622255 \\
C122 & 22.2810737730 & 15.9898035570 & 25.5837500201 \\
H123 & 23.3924382376 & 15.0561927269 & 23.9789874324 \\
H124 & 25.6980598213 & 17.5283416334 & 26.3583357071 \\
C125 & 23.6127510576 & 17.4393268578 & 26.9688614401 \\
C126 & 15.6639067329 & 21.0316049708 & 25.0733205111 \\
C127 & 17.5053677394 & 19.8125278473 & 24.1220232453 \\
C128 & 22.3656886992 & 16.8987387689 & 26.6456104099 \\
H129 & 21.3532386993 & 15.5107226979 & 25.3026808550 \\
H130 & 23.7546444355 & 18.0961610251 & 27.8168129319 \\
H131 & 14.8396327740 & 21.7276115360 & 24.9674114765 \\
C132 & 15.9420825378 & 20.4862364984 & 26.3226225474 \\
C133 & 17.7755760079 & 19.2593108812 & 25.3743347754 \\
H134 & 18.1483175572 & 19.5369667668 & 23.2931993867 \\
C135 & 21.0932728436 & 17.3612273138 & 27.2841218313 \\
H136 & 15.3329416843 & 20.7662160778 & 27.1744124576 \\
C137 & 17.0045593572 & 19.5914816129 & 26.4849234939 \\
H138 & 18.5988372467 & 18.5597175474 & 25.4554725426 \\
C139 & 19.9836483415 & 16.5179800252 & 27.3795404146 \\
C140 & 20.9477262195 & 18.7068017231 & 27.6414375234 \\
C141 & 17.2686942426 & 19.0083355936 & 27.8778293174 \\
\hline & 18.7456110310 & 17.0664072184 & 27.6742824431 \\
20.0439870513 & 15.4549110748 & 27.1896679260 \\
H143 & & & \\
H & &
\end{tabular}




$\begin{array}{llll}\text { H144 } & 21.7762272471 & 19.4014619687 & 27.6270649542 \\ \text { C145 } & 19.6894193171 & 19.1932972783 & 27.9384613220 \\ \text { N146 } & 18.6166558452 & 18.3847386295 & 27.8922189652 \\ \text { H147 } & 17.2509646638 & 19.7863638748 & 28.6382319653 \\ \text { H148 } & 16.5438053866 & 18.2378209880 & 28.1316804417 \\ \text { H149 } & 17.8406742988 & 16.4747882610 & 27.6915211334 \\ \text { H150 } & 19.5063715278 & 20.2358235461 & 28.1613142725\end{array}$

$\mathbf{2}^{3+}$ CBPQT circling glycol chain

-3451.979604 -3451.544953 -3452.501423 -3452.936074

$\begin{array}{llll}\text { O1 } & 24.7525504923 & 11.5235480797 & 20.8998544749 \\ \text { C2 } & 23.9178343975 & 12.5395042743 & 21.2568847222 \\ \text { C3 } & 25.7521174390 & 11.7828677823 & 19.9206748394 \\ \text { C4 } & 22.8169776147 & 12.1841214717 & 22.0542311463 \\ \text { C5 } & 24.0894452286 & 13.8674103092 & 20.8866926156 \\ \text { H6 } & 26.4705702514 & 12.5164493838 & 20.2933978511 \\ \text { H7 } & 25.2850949705 & 12.1526930504 & 19.0052776511 \\ \text { C8 } & 26.4853816803 & 10.4803564450 & 19.6361825899 \\ \text { C9 } & 21.9072521906 & 13.1428424166 & 22.4601778300 \\ \text { H10 } & 22.6931156287 & 11.1460000938 & 22.3362629247 \\ \text { H11 } & 24.9172524944 & 14.1718241041 & 20.2617322440 \\ \text { C12 } & 23.1747833890 & 14.8427829505 & 21.3110722269 \\ \text { O13 } & 27.3152951454 & 10.1880202985 & 20.7417361796 \\ \text { H14 } & 25.7588452669 & 9.6799332158 & 19.4652579358 \\ \text { H15 } & 27.0862230188 & 10.6107528831 & 18.7312474942 \\ \text { C16 } & 22.0827824309 & 14.4847211205 & 22.0895710257 \\ \text { H17 } & 21.0526047996 & 12.8744204644 & 23.0689128976\end{array}$




\begin{tabular}{llll} 
H18 & 23.3488767654 & 15.8698509350 & 21.0212558049 \\
C19 & 28.0902414197 & 9.0131777885 & 20.5738996741 \\
O20 & 21.1480969861 & 15.3676237469 & 22.5586831199 \\
H21 & 28.6498471760 & 9.0551514956 & 19.6346827755 \\
H22 & 27.4432863272 & 8.1307827977 & 20.5613800404 \\
C23 & 29.0716610065 & 8.9015609878 & 21.7327282802 \\
C24 & 21.3695289401 & 16.7411694223 & 22.3044160899 \\
O25 & 30.0688864850 & 9.8947252096 & 21.5794532752 \\
H26 & 28.5372527105 & 9.0329671458 & 22.6786422684 \\
H27 & 29.5222671576 & 7.9039254530 & 21.7194607719 \\
C28 & 20.2881532116 & 17.5210253674 & 23.0587908128 \\
H29 & 21.2941807188 & 16.9418689244 & 21.2342684678 \\
H30 & 22.3632204022 & 17.0306553076 & 22.6554454179 \\
C31 & 31.0654764261 & 9.8535779908 & 22.5848022755 \\
O32 & 20.5596088882 & 18.9131332954 & 22.9859144703 \\
H33 & 19.3132152871 & 17.3480760184 & 22.6065190121 \\
H34 & 20.2643400484 & 17.1852959500 & 24.0980634040 \\
H35 & 31.5379675158 & 8.8663354015 & 22.6158166467 \\
H36 & 30.6283503111 & 10.0664580247 & 23.5652821477 \\
C37 & 32.1290468479 & 10.8955295793 & 22.2656967785 \\
C38 & 21.5155396861 & 19.3392508119 & 23.9462060306 \\
O39 & 31.5469520319 & 12.1822319145 & 22.3638504965 \\
H40 & 32.9502462040 & 10.7892840490 & 22.9817005810 \\
H41 & 32.5170859732 & 10.7279097019 & 21.2563071729 \\
\hline & 22.0015817081 & 20.7255809493 & 23.5361127761 \\
\hline
\end{tabular}




\begin{tabular}{llll} 
C45 & 32.4640749696 & 13.2387138124 & 22.1626841971 \\
O46 & 23.2112692061 & 20.9739875981 & 24.2197021529 \\
H47 & 22.1592441987 & 20.7260621764 & 22.4513676478 \\
H48 & 21.2517901585 & 21.4826106730 & 23.7861167547 \\
H49 & 32.9481169004 & 13.1503746401 & 21.1852509040 \\
H50 & 33.2344154780 & 13.2391509758 & 22.9399932026 \\
C51 & 31.6908058819 & 14.5485449275 & 22.2048242779 \\
C52 & 23.6854940988 & 22.2978909287 & 24.0230811904 \\
O53 & 31.2415200995 & 14.7402432435 & 23.5393525695 \\
H54 & 32.3400177948 & 15.3729600451 & 21.9014312816 \\
H55 & 30.8382657797 & 14.4828995813 & 21.5271398434 \\
C56 & 24.9456250223 & 22.5261324686 & 24.8470975216 \\
H57 & 22.9243504862 & 23.0185182962 & 24.3367989234 \\
H58 & 23.9149721796 & 22.4599352704 & 22.9645662855 \\
C59 & 30.3759989402 & 15.7582215794 & 23.8018986497 \\
O60 & 26.0379665225 & 21.7728380212 & 24.3424511169 \\
H61 & 25.2093257591 & 23.5818744604 & 24.7790474095 \\
H62 & 24.7543144255 & 22.2794905384 & 25.8937141867 \\
C63 & 29.9431574933 & 16.6951861166 & 22.8685188801 \\
C64 & 29.9151342401 & 15.8360247807 & 25.1246569887 \\
C65 & 26.3106284458 & 20.5845380268 & 25.0598540196 \\
H66 & 30.3005538299 & 16.6730982831 & 21.8478856435 \\
C67 & 29.0259031089 & 17.6889650738 & 23.2423367252 \\
\hline & 29.0177853872 & 16.8207830262 & 25.4943586809 \\
H69 & 30.2730488039 & 15.1095397696 & 25.8430479029 \\
25.4077119155 & 19.9786822738 & 25.1502583415
\end{tabular}




\begin{tabular}{llll} 
H72 & 26.6896096339 & 20.8138571642 & 26.0589630011 \\
H73 & 28.6909313780 & 18.3996798499 & 22.4983050400 \\
C74 & 28.5527746988 & 17.7427432165 & 24.5464923449 \\
H75 & 28.6536458100 & 16.8883083285 & 26.5128138163 \\
O76 & 27.5818904493 & 18.6095585983 & 24.9840791200 \\
H77 & 26.9965908164 & 19.6213557638 & 23.2649996527 \\
H78 & 28.2981655399 & 20.3928512863 & 24.2137671736 \\
N79 & 23.5739769985 & 19.7681294851 & 19.2999578741 \\
C80 & 23.3061767968 & 21.0708789293 & 19.4740086234 \\
C81 & 22.5858467710 & 18.8560704042 & 19.2742036302 \\
C82 & 24.9822719957 & 19.2764813539 & 19.2638872731 \\
H83 & 24.1418071179 & 21.7556632707 & 19.4258143194 \\
C84 & 22.0120490947 & 21.4996076652 & 19.7319902743 \\
C85 & 21.2831100628 & 19.2210042957 & 19.5382710733 \\
H86 & 22.8747341364 & 17.8330659660 & 19.0711127624 \\
C87 & 25.2450824739 & 18.4586224238 & 20.5286694325 \\
H88 & 25.6268232269 & 20.1490995733 & 19.1943939047 \\
H89 & 25.0996290700 & 18.6688235195 & 18.3691859554 \\
H90 & 21.8443940610 & 22.5593322737 & 19.8684484586 \\
C91 & 20.9864756731 & 20.5576787962 & 19.8348228975 \\
H92 & 20.5287226856 & 18.4473200343 & 19.5478265829 \\
C93 & 26.0017168224 & 17.2869647208 & 20.4537678815 \\
C94 & 24.7296402484 & 18.8509063806 & 21.7659652288 \\
H95 & 19.6333192897 & 20.9091522199 & 20.3702897511 \\
C96 & 26.2076592847 & 16.5056860626 & 21.5916666360 \\
\hline & 26.4194624930 & 16.9738443451 & 19.5040400210 \\
H97 & 24.1732383585 & 19.7761616731 & 21.8789880939
\end{tabular}




\begin{tabular}{llll} 
C99 & 24.9169295257 & 18.0538212584 & 22.8925076847 \\
C100 & 19.4796055584 & 22.0087191790 & 21.2206593609 \\
C101 & 18.5259648836 & 20.0889008331 & 20.1370841356 \\
C102 & 25.6393045588 & 16.8618872742 & 22.8163750256 \\
H103 & 26.7968517703 & 15.5990687565 & 21.5148449356 \\
H104 & 24.4812946363 & 18.3809345583 & 23.8277402385 \\
H105 & 20.2797355791 & 22.7055761699 & 21.4213049298 \\
C106 & 18.2814131638 & 22.1958756357 & 21.8821857606 \\
C107 & 17.3557220955 & 20.3155184787 & 20.8400276515 \\
H108 & 18.5470920201 & 19.2656497661 & 19.4377395323 \\
C109 & 25.8169733678 & 15.9447898609 & 24.0283856073 \\
N110 & 17.2669513254 & 21.3316912696 & 21.7134342797 \\
H111 & 18.1226134598 & 23.0049945305 & 22.5822855571 \\
H112 & 16.4883746178 & 19.6767312992 & 20.7406470434 \\
N113 & 24.6873905427 & 16.0578986029 & 24.9574118193 \\
H114 & 25.8733936859 & 14.9100491963 & 23.6994520384 \\
H115 & 26.7295913599 & 16.1914187280 & 24.5657878335 \\
C116 & 16.1076476792 & 21.4070886164 & 22.6350981367 \\
C117 & 23.5954307880 & 15.2621027531 & 24.7854855034 \\
C118 & 24.6468800477 & 17.0544043241 & 25.8879262404 \\
H119 & 15.2548618289 & 20.9577282377 & 22.1307661988 \\
H120 & 15.9012785739 & 22.4591900113 & 22.8222752701 \\
C121 & 16.4407077455 & 20.6654750842 & 23.9322331689 \\
C122 & 22.4285426333 & 15.4887705826 & 25.4503008400 \\
H123 & 23.7136393024 & 14.4433456210 & 24.0894854744 \\
H124 & 25.5699419438 & 17.6048967543 & 26.0238115148 \\
\hline & 23.5054351484 & 17.3436384672 & 26.5772535684
\end{tabular}




$\begin{array}{llll}\text { C126 } & 15.4896936677 & 20.6719251044 & 24.9606754647 \\ \text { C127 } & 17.6373012487 & 19.9839640623 & 24.1243894189 \\ \text { C128 } & 22.2961013375 & 16.6024433208 & 26.3448398985 \\ \text { H129 } & 21.6093984691 & 14.8141425658 & 25.2540185277 \\ \text { H130 } & 23.5517395304 & 18.1434299088 & 27.3015614133 \\ \text { H131 } & 14.5518964586 & 21.1995516856 & 24.8268687023 \\ \text { C132 } & 15.7364557594 & 20.0062985219 & 26.1553681046 \\ \text { C133 } & 17.8802196379 & 19.3171388467 & 25.3272927849 \\ \text { H134 } & 18.4120135299 & 19.9430164366 & 23.3655254139 \\ \text { C135 } & 21.0410721503 & 16.9711504390 & 26.9083787033 \\ \text { H136 } & 14.9882522695 & 20.0223356189 & 26.9401528176 \\ \text { C137 } & 16.9399900985 & 19.3176503939 & 26.3510454865 \\ \text { H138 } & 18.8202740130 & 18.7941059674 & 25.4415153980 \\ \text { C139 } & 19.8423671547 & 16.2121703353 & 26.6867593117 \\ \text { C140 } & 20.8615000327 & 18.1723947937 & 27.6713817090 \\ \text { C141 } & 17.1872676486 & 18.5884408053 & 27.6764745111 \\ \text { C142 } & 18.6241295005 & 16.7088558826 & 27.0434944309 \\ \text { H143 } & 19.8601813272 & 15.2394291869 & 26.2190312177 \\ \text { H144 } & 21.6966724011 & 18.7837716453 & 27.9779432313 \\ \text { C145 } & 19.6146884965 & 18.6178784970 & 27.9948641751 \\ \text { N146 } & 18.4942609537 & 17.9223227115 & 27.6517154228 \\ \text { H147 } & 17.1790378661 & 19.2928754698 & 28.5072831455 \\ \text { H148 } & 16.4195809862 & 17.8347632322 & 27.8473779482 \\ \text { H149 } & 17.7038175222 & 16.1770341773 & 26.8413455683 \\ & 19.4548976601 & 19.5525200102 & 28.5158828095\end{array}$

$\mathbf{2}^{2+} \mathrm{CBPQT}$ circling glycol chain 


$\begin{array}{llll}-3452.138738-3451.919671 & -3452.880485 & -3453.099552 \\ \text { O1 } & 24.7200472822 & 11.5377436444 & 20.9014824304 \\ \text { C2 } & 23.8746112714 & 12.5545610188 & 21.2329796541 \\ \text { C3 } & 25.7185095482 & 11.7735165880 & 19.9170468165 \\ \text { C4 } & 22.7718718476 & 12.1966438949 & 22.0251499480 \\ \text { C5 } & 24.0359750183 & 13.8799843848 & 20.8468033912 \\ \text { H6 } & 26.4422231683 & 12.5093380886 & 20.2751304027 \\ \text { H7 } & 25.2537136665 & 12.1285399496 & 18.9946957481 \\ \text { C8 } & 26.4396370856 & 10.4582597146 & 19.6592265186 \\ \text { C9 } & 21.8530448991 & 13.1519446737 & 22.4154776918 \\ \text { H10 } & 22.6554332692 & 11.1602343981 & 22.3167342119 \\ \text { H11 } & 24.8660140279 & 14.1836775103 & 20.2238245637 \\ \text { C12 } & 23.1101682078 & 14.8543285418 & 21.2543647319 \\ \text { O13 } & 27.2656953124 & 10.1781778952 & 20.7712868152 \\ \text { H14 } & 25.7035775377 & 9.6632121293 & 19.5045141783 \\ \text { H15 } & 27.0427304841 & 10.5626128799 & 18.7523697801 \\ \text { C16 } & 22.0203418505 & 14.4923823959 & 22.0356770950 \\ \text { H17 } & 20.9963908319 & 12.8838902161 & 23.0216385385 \\ \text { H18 } & 23.2693118063 & 15.8812919034 & 20.9499676457 \\ \text { C19 } & 28.0150345606 & 8.9831564318 & 20.6327147993 \\ \text { O20 } & 21.0737448567 & 15.3613753310 & 22.4992306787 \\ \text { H21 } & 28.5603710925 & 8.9820550429 & 19.6843321374 \\ \text { H22 } & 27.3520383569 & 8.1129621966 & 20.6605023659 \\ \text { C23 } & 29.0137825812 & 8.8943638664 & 21.7786328657 \\ \text { H26 } & 21.2911841013 & 16.7498271663 & 22.3135843490 \\ & 30.0081849175 & 9.8826366006 & 21.5836944999 \\ \text { O25 } & 2857752895 & 9.0514349351 & 22.7297614131\end{array}$




\begin{tabular}{llll} 
H27 & 29.4613719853 & 7.8952606809 & 21.7829233083 \\
C28 & 20.2576771573 & 17.4647767666 & 23.1895407790 \\
H29 & 21.1546928330 & 17.0280758890 & 21.2667014701 \\
H30 & 22.3047722800 & 17.0132586391 & 22.6258182403 \\
C31 & 31.0193518842 & 9.8761589613 & 22.5742872441 \\
O32 & 20.4761094599 & 18.8622877398 & 23.1624437280 \\
H33 & 19.2551491905 & 17.2830289089 & 22.8053702438 \\
H34 & 20.3249505843 & 17.0773731600 & 24.2094985533 \\
H35 & 31.5236731854 & 8.9047314740 & 22.6016213089 \\
H36 & 30.5912235525 & 10.0776988735 & 23.5608025562 \\
C37 & 32.0417245140 & 10.9525475700 & 22.2340310376 \\
C38 & 21.5075492923 & 19.3017269110 & 24.0285915268 \\
O39 & 31.4182814551 & 12.2192058938 & 22.3437458660 \\
H40 & 32.8811295274 & 10.8761242416 & 22.9324381806 \\
H41 & 32.4137482905 & 10.7970969438 & 21.2166760994 \\
C42 & 21.9535831973 & 20.6713695050 & 23.5339079793 \\
H43 & 22.3614334155 & 18.6223446374 & 23.9973699777 \\
H44 & 21.1515848462 & 19.3600887983 & 25.0605768119 \\
C45 & 32.2914501905 & 13.3055248187 & 22.1084572163 \\
O46 & 23.1351920429 & 21.0109343587 & 24.2320829337 \\
H47 & 22.1378091838 & 20.6062959545 & 22.4581693148 \\
H48 & 21.1662837101 & 21.4105039658 & 23.7149738839 \\
H49 & 32.7476478886 & 13.2264381713 & 21.1168085193 \\
C50 & 33.0854517273 & 13.3376412969 & 22.8609083190 \\
\hline 32 & 23.5982648873 & 22.3150661311 & 23.9239426398 \\
\hline
\end{tabular}




\begin{tabular}{|c|c|c|c|}
\hline H54 & 32.0921865286 & 15.4303011712 & 21.8318283808 \\
\hline H55 & 30.6051467667 & 14.4929230597 & 21.5180020284 \\
\hline C56 & 24.8385716831 & 22.6232436175 & 24.7523921977 \\
\hline H57 & 22.8233313582 & 23.0529335686 & 24.1546753195 \\
\hline H58 & 23.8504152866 & 22.3847872432 & 22.8613819095 \\
\hline C59 & 30.2172318563 & 15.8127884115 & 23.7941442782 \\
\hline O60 & 25.9563852752 & 21.8587596136 & 24.3259805750 \\
\hline H61 & 25.0915164820 & 23.6739990494 & 24.6106771627 \\
\hline H62 & 24.6314250731 & 22.4493874732 & 25.8106614089 \\
\hline C63 & 29.7574682135 & 16.7410048628 & 22.8651093927 \\
\hline C64 & 29.8053721354 & 15.9126353021 & 25.1317755382 \\
\hline C65 & 26.1700543229 & 20.6599609498 & 25.0482533618 \\
\hline H66 & 30.0755492721 & 16.7025122001 & 21.8319412541 \\
\hline C67 & 28.8626624155 & 17.7470614154 & 23.2581221120 \\
\hline C68 & 28.9273293409 & 16.9086313544 & 25.5197474446 \\
\hline H69 & 30.1853996004 & 15.1947907258 & 25.8475516842 \\
\hline $\mathrm{C} 70$ & 27.2581839317 & 19.8962730709 & 24.2978797195 \\
\hline H71 & 25.2560812578 & 20.0655201746 & 25.0860695215 \\
\hline $\mathrm{H} 72$ & 26.5032745025 & 20.8768026917 & 26.0666206195 \\
\hline $\mathrm{H} 73$ & 28.5062096926 & 18.4480026585 & 22.5149696186 \\
\hline C74 & 28.4327379674 & 17.8199740655 & 24.5763292600 \\
\hline $\mathrm{H} 75$ & 28.6005349322 & 16.9928430741 & 26.5494074252 \\
\hline $\mathrm{O} 76$ & 27.4843968169 & 18.7000183597 & 25.0335954012 \\
\hline $\mathrm{H} 77$ & 26.9043078892 & 19.6752186688 & 23.2913896047 \\
\hline H78 & 28.1765490359 & 20.4847263734 & 24.2475496337 \\
\hline N79 & 23.7821995027 & 19.8827544728 & 19.3152360611 \\
\hline $\mathrm{C} 80$ & 23.4713234652 & 21.1434883268 & 19.7304708053 \\
\hline
\end{tabular}




\begin{tabular}{llll} 
C81 & 22.7728710996 & 18.9901023403 & 19.0909185830 \\
C82 & 25.1676591518 & 19.3890820897 & 19.3292641165 \\
H83 & 24.3005545051 & 21.8321798875 & 19.8237123517 \\
C84 & 22.1886705825 & 21.5095440649 & 20.0164916050 \\
C85 & 21.4748189559 & 19.2945986601 & 19.3550339262 \\
H86 & 23.0718500876 & 18.0267379848 & 18.6989524277 \\
C87 & 25.3708616026 & 18.4859373261 & 20.5477736962 \\
H88 & 25.8301733816 & 20.2527540413 & 19.3590934601 \\
H89 & 25.3522330584 & 18.8367313632 & 18.4088650425 \\
H90 & 22.0243096648 & 22.5299499066 & 20.3306514005 \\
C91 & 21.1141071941 & 20.5613033298 & 19.9258040004 \\
H92 & 20.7347200811 & 18.5391909902 & 19.1361301829 \\
C93 & 26.1161890092 & 17.3091761173 & 20.4472266590 \\
C94 & 24.7925672168 & 18.8118987388 & 21.7757266477 \\
C95 & 19.8081652089 & 20.8211150235 & 20.4273501071 \\
C96 & 26.2661486331 & 16.4702882314 & 21.5515382513 \\
H97 & 26.5716297241 & 17.0397881729 & 19.5010636818 \\
H98 & 24.2195679808 & 19.7257481608 & 21.8974785965 \\
C99 & 24.9400498668 & 17.9679073559 & 22.8738599595 \\
C100 & 19.4734813844 & 22.0408450616 & 21.0996159554 \\
C101 & 18.7497114032 & 19.8570744016 & 20.3545652388 \\
C102 & 25.6652517979 & 16.7816371983 & 22.7725582411 \\
H103 & 26.8490746710 & 15.5607883802 & 21.4583335466 \\
H104 & 24.4760671775 & 18.2545867543 & 23.8086324679 \\
C105 & 20.1737228256 & 22.8594569889 & 21.1750013190 \\
\hline & 18.2639445385 & 22.1984773429 & 21.7088426570 \\
\hline
\end{tabular}




\begin{tabular}{llll} 
H108 & 18.8614006994 & 18.9237889544 & 19.8237984318 \\
C109 & 25.8272151908 & 15.8269417837 & 23.9554744335 \\
N110 & 17.3113530085 & 21.2279971387 & 21.6690255521 \\
H111 & 18.0113310797 & 23.0927120399 & 22.2628842875 \\
H112 & 16.7519320906 & 19.3583579299 & 20.9546240069 \\
N113 & 24.7188160524 & 15.9537187330 & 24.9081440114 \\
H114 & 25.8350526802 & 14.8007524649 & 23.5971327345 \\
H115 & 26.7604278078 & 16.0233869342 & 24.4809579117 \\
C116 & 16.1568806520 & 21.2965567663 & 22.5737393766 \\
C117 & 23.6077031145 & 15.1828610599 & 24.7494637548 \\
C118 & 24.7089293907 & 16.9513304918 & 25.8383311419 \\
H119 & 15.3179351592 & 20.7946660830 & 22.0926884121 \\
H120 & 15.9002796867 & 22.3450305580 & 22.7188312211 \\
C121 & 16.4874989339 & 20.6261896183 & 23.9110326455 \\
C122 & 22.4565094646 & 15.4279983295 & 25.4343384837 \\
H123 & 23.6936622073 & 14.3691086568 & 24.0440104733 \\
H124 & 25.6368989824 & 17.4998228563 & 25.9518146578 \\
C125 & 23.5839697763 & 17.2552736169 & 26.5476382508 \\
C126 & 15.5298556710 & 20.6101305855 & 24.9333749534 \\
C127 & 17.7140780781 & 20.0108217603 & 24.1310333604 \\
C128 & 22.3563843610 & 16.5396842227 & 26.3342139506 \\
H129 & 21.6192779793 & 14.7741021064 & 25.2415179108 \\
H130 & 23.6543366373 & 18.0563642290 & 27.2688117895 \\
H131 & 14.5691307889 & 21.0903439583 & 24.7818754960 \\
C132 & 15.7957584512 & 19.9766140038 & 26.1431179319 \\
\hline 17.9758644986 & 19.3728437908 & 25.3444623291 \\
\hline & 18.4932655648 & 19.9958652713 & 23.3775136831
\end{tabular}




$\begin{array}{llll}\text { C135 } & 21.1146189338 & 16.9363337589 & 26.9093989783 \\ \text { H136 } & 15.0400687199 & 19.9705503159 & 26.9213286178 \\ \text { C137 } & 17.0261435688 & 19.3425165270 & 26.3586753576 \\ \text { H138 } & 18.9380672492 & 18.8943910909 & 25.4708649949 \\ \text { C139 } & 19.8992398264 & 16.1994808136 & 26.7046863373 \\ \text { C140 } & 20.9639367216 & 18.1497931421 & 27.6590258318 \\ \text { C141 } & 17.2929387110 & 18.6338000736 & 27.6914359944 \\ \text { C142 } & 18.6933080282 & 16.7268270040 & 27.0588938568 \\ \text { H143 } & 19.89498855953 & 15.2212205494 & 26.2479604237 \\ \text { H144 } & 21.8138718077 & 18.7497684217 & 27.9484180966 \\ \text { C145 } & 19.7276018192 & 18.6247301126 & 27.9829822821 \\ \text { N146 } & 18.5911075576 & 17.9485168123 & 27.6548545315 \\ \text { H147 } & 17.3116454966 & 19.3494526306 & 28.5118995023 \\ \text { H148 } & 16.5200997879 & 17.8926997328 & 27.8915451069 \\ \text { H149 } & 17.7609909746 & 16.2128952881 & 26.8663865850 \\ \text { H150 } & 19.5900876196 & 19.5698120881 & 28.4911378058\end{array}$

$2^{1+}$ CBPQT circling glycol chain

$\begin{array}{llll}-3452.269082 & -3452.153287 & -3453.123480 & -3453.239274\end{array}$

$\begin{array}{llll}\text { O1 } & 24.7227782513 & 11.5405764494 & 20.8804341920 \\ \text { C2 } & 23.8681936129 & 12.5483066734 & 21.2265703830 \\ \text { C3 } & 25.7431036366 & 11.8162754304 & 19.9301843706 \\ \text { C4 } & 22.7490372801 & 12.1700254993 & 21.9836477596 \\ \text { C5 } & 24.0416668798 & 13.8841214942 & 20.8848492864 \\ \text { H6 } & 26.4564257929 & 12.5395061969 & 20.3317579529 \\ \text { H7 } & 25.2982756023 & 12.2057542291 & 19.0115447357 \\ \text { C8 } & 26.4763755429 & 10.5155492587 & 19.6366180538\end{array}$




\begin{tabular}{llll} 
C9 & 21.8247195535 & 13.1173710024 & 22.3836275365 \\
H10 & 22.6248971259 & 11.1260600577 & 22.2435324847 \\
H11 & 24.8889523493 & 14.2047600782 & 20.2952421328 \\
C12 & 23.1107574991 & 14.8482417243 & 21.2999477518 \\
O13 & 27.2840962860 & 10.1962092207 & 20.7517335553 \\
H14 & 25.7477060877 & 9.7231336052 & 19.4389214698 \\
H15 & 27.0952382971 & 10.6559317192 & 18.7453983089 \\
C16 & 22.0022443218 & 14.4671978142 & 22.0455743702 \\
H17 & 20.9575362070 & 12.8362264870 & 22.9684496578 \\
H18 & 23.2812877563 & 15.8845656380 & 21.0362914210 \\
C19 & 28.0363248505 & 9.0075835878 & 20.5805112533 \\
O20 & 21.0419945116 & 15.3269520021 & 22.4931214996 \\
H21 & 28.5808623227 & 9.0335848783 & 19.6318683915 \\
H22 & 27.3750855110 & 8.1355609153 & 20.5848977434 \\
C23 & 29.0366808524 & 8.8910708178 & 21.7226371650 \\
C24 & 21.3174513913 & 16.7178569878 & 22.4391035916 \\
O25 & 30.0305940567 & 9.8830100860 & 21.5473361730 \\
H26 & 28.5195754254 & 9.0290386784 & 22.6772614972 \\
H27 & 29.4845539177 & 7.8922917977 & 21.7056800772 \\
C28 & 20.2814765257 & 17.4051716391 & 23.3313077742 \\
H29 & 21.2288849994 & 17.0924560379 & 21.4164044102 \\
H30 & 22.3259497944 & 16.9079495957 & 22.8108213058 \\
C31 & 31.0356134007 & 9.8667543704 & 22.5431318286 \\
H33 & 20.4863916063 & 18.8054293598 & 23.3017870947 \\
\hline & 20.3596927456 & 17.0199344269 & 24.3497019417 \\
H3 3.5505755751 & 8.9003187377 & 22.5537841534
\end{tabular}




\begin{tabular}{|c|c|c|c|}
\hline H36 & 30.5991008534 & 10.0438914396 & 23.5303186051 \\
\hline C37 & 32.0467266991 & 10.9621382000 & 22.2305739002 \\
\hline C38 & 21.5042870948 & 19.2650386528 & 24.1782600456 \\
\hline O39 & 31.4068754807 & 12.2191746491 & 22.3585230999 \\
\hline $\mathrm{H} 40$ & 32.8832442605 & 10.8830563478 & 22.9323248022 \\
\hline H41 & 32.4267682860 & 10.8300784660 & 21.2128930228 \\
\hline $\mathrm{C} 42$ & 21.9689781139 & 20.6010573366 & 23.6141794871 \\
\hline H43 & 22.3458214754 & 18.5704162332 & 24.2080101989 \\
\hline H44 & 21.1210895498 & 19.3781046838 & 25.1953138333 \\
\hline $\mathrm{C} 45$ & 32.2659905119 & 13.3198632774 & 22.1387642082 \\
\hline $\mathrm{O} 46$ & 23.1284740036 & 21.0051399937 & 24.3150893148 \\
\hline $\mathrm{H} 47$ & 22.1831580514 & 20.4669281089 & 22.5495941654 \\
\hline H48 & 21.1721489235 & 21.3448273490 & 23.7219027430 \\
\hline H49 & 32.7321986910 & 13.2559989520 & 21.1507206361 \\
\hline H50 & 33.0531187469 & 13.3548694535 & 22.8984876169 \\
\hline C51 & 31.4372921880 & 14.5949648374 & 22.2019565322 \\
\hline C52 & 23.5841138517 & 22.2841512433 & 23.9122663622 \\
\hline O53 & 31.0074327940 & 14.7724378956 & 23.5440307642 \\
\hline H54 & 32.0449059780 & 15.4454539104 & 21.8840432548 \\
\hline H55 & 30.5730232762 & 14.4947999120 & 21.5440184023 \\
\hline C56 & 24.8326665846 & 22.6565486978 & 24.7012777783 \\
\hline H57 & 22.8077448768 & 23.0351213263 & 24.0939476749 \\
\hline H58 & 23.8283346444 & 22.2787020270 & 22.8450679547 \\
\hline C59 & 30.1735557402 & 15.8144300554 & 23.8268220372 \\
\hline O60 & 25.9573086542 & 21.8891692474 & 24.3009782086 \\
\hline H61 & 25.0673663719 & 23.7014488470 & 24.4967801976 \\
\hline H62 & 24.6454267666 & 22.5404521918 & 25.7713143184 \\
\hline
\end{tabular}




\begin{tabular}{llll} 
C63 & 29.7248925392 & 16.7464197427 & 22.8975373999 \\
C64 & 29.7669283304 & 15.9232121247 & 25.1651661489 \\
C65 & 26.1610807724 & 20.6949469500 & 25.0374358786 \\
H66 & 30.0330291707 & 16.6970616629 & 21.8618301031 \\
C67 & 28.8503053325 & 17.7702068608 & 23.2912874686 \\
C68 & 28.9087409657 & 16.9353131093 & 25.5535657422 \\
H69 & 30.1346446182 & 15.1984812921 & 25.8806038363 \\
C70 & 27.2418804403 & 19.9162882258 & 24.2924389401 \\
H71 & 25.2440117307 & 20.1067057036 & 25.0857394277 \\
H72 & 26.5013245577 & 20.9242458216 & 26.0510228522 \\
H73 & 28.5007225544 & 18.4718543613 & 22.5458246888 \\
C74 & 28.4286686772 & 17.8561215694 & 24.6110823889 \\
H75 & 28.5839982764 & 17.0256952561 & 26.5832630056 \\
O76 & 27.5180540287 & 18.7655542862 & 25.0783155678 \\
H77 & 26.8625317482 & 19.6371626952 & 23.3099302780 \\
H78 & 28.1447698764 & 20.5216665264 & 24.1837970364 \\
N79 & 23.8666700378 & 19.7680619391 & 19.3399733036 \\
C80 & 23.5782326575 & 21.0269931411 & 19.7773773256 \\
C81 & 22.8419725050 & 18.8921866805 & 19.1183508596 \\
C82 & 25.2427799378 & 19.2477738354 & 19.3624562882 \\
H83 & 24.4183475206 & 21.7024927063 & 19.8695832629 \\
C84 & 22.3048412676 & 21.4050373514 & 20.0892080669 \\
C85 & 21.5511250116 & 19.2111245918 & 19.4018495231 \\
H86 & 23.1239136431 & 17.9271866601 & 18.7179294909 \\
C87 & 25.4225830761 & 18.3593259360 & 20.5949503812 \\
H88 & 25.9210121758 & 20.0994246415 & 19.3793405840 \\
\hline & 25.4169841723 & 18.6781354462 & 18.4506235394
\end{tabular}




\begin{tabular}{llll} 
H90 & 22.1555756330 & 22.4240472452 & 20.4150595008 \\
C91 & 21.2164040968 & 20.4730155955 & 19.9993763451 \\
H92 & 20.7992385857 & 18.4647643282 & 19.1912424433 \\
C93 & 26.1691570161 & 17.1809859824 & 20.5243431507 \\
C94 & 24.8168311789 & 18.6990182433 & 21.8059143147 \\
C95 & 19.9230558858 & 20.7433253420 & 20.5261885557 \\
C96 & 26.2956486043 & 16.3592728770 & 21.6436815509 \\
H97 & 26.6428328821 & 16.8983620705 & 19.5908059827 \\
H98 & 24.2411796427 & 19.6130590180 & 21.9019746911 \\
C99 & 24.9344051338 & 17.8671621448 & 22.9168847703 \\
C100 & 19.6313224293 & 21.9353106012 & 21.2651758607 \\
C101 & 18.8357701496 & 19.8164606325 & 20.4120789405 \\
C102 & 25.6662102280 & 16.6826668133 & 22.8471529191 \\
H103 & 26.8817856779 & 15.4493867580 & 21.5763478445 \\
H104 & 24.4410581239 & 18.1506761104 & 23.8390287402 \\
H105 & 20.3652864027 & 22.7175426266 & 21.3939513046 \\
C106 & 18.4260771776 & 22.1069367804 & 21.8786619976 \\
C107 & 17.6457369190 & 20.0561951079 & 21.0326475923 \\
H108 & 18.9183399261 & 18.9055581857 & 19.8385748832 \\
C109 & 25.7910157996 & 15.7527309296 & 24.0545395664 \\
N110 & 17.4354590148 & 21.1781699635 & 21.7761134175 \\
H111 & 18.2040394078 & 22.9777146755 & 22.4812628935 \\
H112 & 16.8173328178 & 19.3619422580 & 20.9772764942 \\
N113 & 24.6975773501 & 15.8869503927 & 25.0022001370 \\
H114 & 25.8031158173 & 14.7220041221 & 23.7038364951 \\
H116 & 16.2548152965 & 21.2661662505 & 22.6446589277 \\
\hline
\end{tabular}




\begin{tabular}{llll} 
C117 & 23.5424724058 & 15.1493869751 & 24.8197448593 \\
C118 & 24.6393439663 & 16.9592689524 & 25.8747492415 \\
H119 & 15.4217478470 & 20.7877579848 & 22.1307251839 \\
H120 & 16.0165099965 & 22.3192973534 & 22.7874428772 \\
C121 & 16.5269280594 & 20.5826112443 & 23.9866936583 \\
C122 & 22.3849590583 & 15.4311038014 & 25.4433721275 \\
H123 & 23.6374597233 & 14.3065189664 & 24.1475245548 \\
H124 & 25.5695555476 & 17.5025450087 & 25.9942895481 \\
C125 & 23.5108207410 & 17.3175846507 & 26.5181295679 \\
C126 & 15.5424599220 & 20.5987741601 & 24.9831310895 \\
C127 & 17.7289571706 & 19.9315793065 & 24.2382083309 \\
C128 & 22.2381380005 & 16.5954042994 & 26.3331211111 \\
H129 & 21.5488328375 & 14.7761456204 & 25.2458077903 \\
H130 & 23.5717257372 & 18.1597332004 & 27.1931329471 \\
H131 & 14.5987870695 & 21.1030257133 & 24.8033615782 \\
C132 & 15.7647436744 & 19.9680065763 & 26.2033085990 \\
C133 & 17.9467226150 & 19.2977227421 & 25.4628016018 \\
H134 & 18.5231970556 & 19.8877638342 & 23.5025239675 \\
C135 & 21.0539337561 & 16.9664715185 & 26.9000923815 \\
H136 & 14.9915624462 & 19.9878018472 & 26.9645108676 \\
C137 & 16.9738120098 & 19.3068748045 & 26.4556200181 \\
H138 & 18.8885283201 & 18.7898109439 & 25.6255714749 \\
C139 & 19.7947366221 & 16.2130810358 & 26.7345176788 \\
C140 & 20.8733495734 & 18.1808415426 & 27.7175947948 \\
C141 & 17.2147770405 & 18.6054759722 & 27.7968307148 \\
H143 & 18.6138124525 & 16.6954910011 & 27.1595555264 \\
\hline 19.7932074794 & 15.2376636431 & 26.2688001411 \\
\hline
\end{tabular}




$\begin{array}{llll}\text { H144 } & 21.7237559786 & 18.7747427306 & 28.0196062744 \\ \text { C145 } & 19.6555136511 & 18.6033756260 & 28.1046125884 \\ \text { N146 } & 18.4878610810 & 17.9025987565 & 27.8334673843 \\ \text { H147 } & 17.2036411315 & 19.3390551019 & 28.6041389984 \\ \text { H148 } & 16.4132595568 & 17.8885315727 & 27.9838483489 \\ \text { H149 } & 17.6873781762 & 16.1526903732 & 27.0158237197 \\ \text { H150 } & 19.5207604809 & 19.5145814861 & 28.6746142106\end{array}$

$2^{0} \mathrm{CBPQT}$ circling glycol chain

$-3452.394624-3452.325341-3453.305409-3453.374692$

O1 $6.4240351 .074150-5.287101$

C2 $5.1998560 .883213-4.708588$

C3 $7.4603520 .144848-5.002758$

C4 $4.1648801 .700345-5.186791$

C5 $4.931521-0.042256-3.706340$

H6 $7.7522090 .206724-3.951594$

H7 7.125144 -0.868834 -5.233474

C8 $8.6573360 .494788-5.875048$

C9 $2.8884371 .585037-4.666901$

H10 4.385979 2.417950 -5.967156

H11 $5.707103-0.686655-3.317249$

C12 $3.638544-0.155571-3.172697$

O13 9.259828 1.668633 -5.367144

H14 $8.3226990 .639993-6.906866$

H15 9.365815 -0.338555 -5.848993

C16 2.616620 0.656327 -3.651068

H17 2.083345 2.213426 -5.027117 
H18 3.461224 -0.872354 -2.379491

C19 10.388979 2.090039 -6.112915

O20 1.330554 0.628257-3.196450

H21 11.087798 1.259467 -6.251901

H22 10.079931 2.458161 -7.095810

C23 11.093254 3.205313 -5.351746

C24 1.071846-0.028520 - 1.960156

O25 11.723772 2.641195-4.217552

H26 10.362616 3.963216 -5.051781

H27 11.833966 3.672365 -6.008726

C28 -0.293000 $0.473854-1.478621$

H29 $1.050043-1.112637-2.088224$

H30 1.849616 0.234788 - 1.242939

C31 12.429014 3.580025 -3.427504

O32 -0.641669-0.143763-0.257867

H33 -1.059553 0.197807 -2.201341

H34 -0.265087 1.560077-1.375634

H35 13.217174 4.063832 -4.013560

H36 11.749396 4.349818 -3.049776

C37 13.062143 2.843524 -2.255142

C38 -0.002319 0.3857220.890697

O39 12.028641 2.333121-1.433104

H40 13.691164 3.542168 -1.694462

H41 13.688818 2.028469 -2.629821

C42 $0.015251-0.7375991 .918227$

H43 1.0231880 .6876290 .673818

H44 -0.538646 1.259983 1.267722 
C45 12.500469 1.656839-0.284822

O46 $0.815462-0.3264923 .013035$

H47 0.428486 -1.631093 1.443764

H48 -1.005639 -0.959550 2.246319

H49 13.179993 0.846213 -0.565620

H50 13.029365 2.344765 0.381677

C51 11.3062331 .0564280 .440628

C52 0.931725 -1.339261 3.994741

O53 10.5443872 .1229920 .987545

H54 11.653613 0.392327 1.235438

H55 10.701859 0.491779 -0.270730

C56 1.834566 -0.862753 5.125304

H57 -0.055518 - 1.5825584 .404024

H58 1.363122 -2.243187 3.554238

C59 9.353544 1.8274361.584506

O60 $3.202192-0.8472524 .746656$

H61 1.745198 -1.564949 5.954182

H62 1.516572 0.126050 5.465896

C63 8.8447390 .5440091 .747595

C64 8.622502 2.926005 2.061057

C65 3.6450380 .3627104 .157670

H66 9.395376 -0.323453 1.409870

C67 7.592259 0.352493 2.351293

C68 7.390387 2.737190 2.658087

H69 9.039065 3.9183391 .943624

C70 5.068257 0.106740 3.667271

H71 3.0014720 .6494783 .325798 
H72 3.6598191 .1650154 .901527

H73 7.209226 -0.653835 2.450990

C74 6.855915 1.445927 2.785897

H75 6.812696 3.578615 3.021051

O76 5.594746 1.377084 3.309392

H77 $5.047258-0.5618602 .806081$

H78 $5.665668-0.3337434 .468893$

N79 2.880585 -4.2162840.093896

C80 2.079874 -4.302000 1.224101

C81 2.247196 -3.947530 -1.113624

C82 4.299658 -3.911980 0.206476

H83 2.602957 -4.555342 2.137948

C84 $0.745918-4.1220841 .194682$

C85 $0.922391-3.755068-1.219087$

H86 2.900268 -3.930537-1.977539

C87 4.569687 -2.406627 0.139975

H88 4.660996 -4.324228 1.148927

H89 4.834725 -4.410404-0.603795

H90 0.204695 -4.265364 2.119332

C91 0.034559-3.746247 -0.042788

H92 $0.520507-3.608702-2.211537$

C93 $5.775838-1.920229-0.375379$

C94 3.607826-1.493251 0.570895

C95 -1.281913-3.400755 -0.095708

C96 $6.007089-0.549454-0.466287$

H97 $6.530846-2.618262-0.721690$

H98 2.661768-1.8433120.969508 
C99 $3.842944-0.1215050 .476387$

C100 -2.168502 -3.354486 1.078428

C101 -1.977161 -3.013486-1.334355

C102 $5.0352220 .365991-0.054155$

H103 6.944829 -0.185481 -0.874366

H104 3.0809350 .5702980 .813937

H105 - $1.794551-3.6091442 .060127$

C106 -3.457044 -2.977153 0.988922

C107 -3.274639-2.660799-1.347425

H108 - $1.452083-2.995879-2.278230$

C109 $5.2834331 .866145-0.206864$

N110 -4.067856 -2.650991 -0.210202

H111 -4.096796 -2.918965 1.860584

H112 -3.777092 -2.361175 -2.258594

N113 4.062923 2.654955-0.150412

H114 $5.7551612 .047120-1.172239$

H115 5.9636552 .2091860 .573977

C116 -5.292747 - $1.866121-0.206333$

C117 3.293253 2.789106 -1.293551

C118 3.442286 2.8895341.065049

H1 19 -5.834197 -2.063174 -1.133413

H120 -5.916784 -2.205230 0.621805

C121 -5.023697-0.364087-0.077869

C122 2.005822 $3.179110-1.274796$

H123 $3.8078182 .565740-2.219133$

H124 4.069479 2.731562 1.934794

C125 2.1521413.2626331.167687 
C126 -6.090546 $0.543541-0.050850$

C127-3.722852 0.123873 -0.005293

C128 1.290766 3.449492-0.014502

H129 $1.5033323 .260555-2.227603$

H130 1.7625293 .4267812 .162256

H131 -7.111002 0.178205 -0.110086

C132 -5.853780 1.9113970.044337

C133 -3.488342 1.4979720 .085813

H134 -2.866028 -0.540784 -0.032976

C135 -0.024242 3.8040510 .048927

H136 -6.690878 2.6024010.059876

C137 -4.544062 2.403808 0.113019

H138 -2.464632 1.8485990 .125077

C139-0.872766 4.017628 -1.138855

C140 -0.773097 3.9841541 .306958

C141 -4.290344 3.9119550.206723

C142 -2.202750 4.189478 -1.045389

H143 -0.436712 4.046076 -2.127542

H144 -0.263313 3.9745422 .259131

C145 -2.108685 4.150143 1.321749

N146 -2.874283 4.2418350.167611

H147 -4.708570 4.3022791 .136010

H148 -4.788436 4.418814 -0.622024

H149 -2.827459 $4.318471-1.920850$

H150 -2.663216 4.243071 2.247617 


\section{References}

S1. (a) Odell, B.; Reddington, M. V.; Slawin, A. M. Z.; Spencer, N.; Stoddart, J. F.; Williams, D. J. Angew. Chem. Int. Ed. Engl. 1988, 27, 1547-1550. (b) Sue, C.-H.; Basu, S.; Fahrenbach, A.; Shveyd, A. K.; Dey, S. K.; Botros, Y. Y.; Stoddart, J. F. Chem. Sci. 2010, 1, 119-125. (c) Barnes, J. C.; Juríček, M.; Vermeulen, N. A.; Dale, E. J.; Stoddart, J. F.; J. Org. Chem. 2013, 78, 11962-11969.

S2. (a) Helgeson, R. C.; Tarnowski, T. L.; Timko, J. M.; Cram, D. J.; J. Am. Chem. Soc. 1977, 99, 6411-6418. (b) Anelli, P.-L.; Ashton, P. R.; Ballardini, R.; Balzani, V.; Delgado, M.; Gandolfi, M. T.; Goodnow, T. T.; Kaifer, A. E.; Philp, D.; Pietraszkiewicz, M.; Prodi, L.; Reddington, M. V.; Slawin, A. M. Z.; Spencer, N.; Stoddart, J. F.; Vicent, C.; Williams D. J. J. Am. Chem. Soc. 1992, 114, 193-218.

S3. Hamilton, D. G.; Davies, J. E.; Prodi, L.; Sanders, J. K. M. Chem. Eur. J. 1998, 4, 608-620.

S4. (a) Ashton, P. R.; Brown, C. L.; Chrystal, E. J. T.; Goodnow, T.; Kaifer, A. E.; Parry, K. P.; Philp, D.; Slawin, A. M. Z.; Spencer, N.; Stoddart, J. F.; Williams, D. J. J. Chem. Soc., Chem. Commun. 1991, 9, 634-639. (b) Miljanić, O. Š.; Stoddart, J. F. Proc. Natl. Acad. Sci. USA 2007, 104, 12966-12970.

S5. Ashton, P. R.; Goodnow, T. T.; Kaifer, A. E.; Reddington, M. V.; Slawin, A. M. Z.; Spencer, N.; Stoddart, J. F.; Vicent, C.; Williams, D. J. Angew. Chem. Int. Ed. Engl. 1989, 28, 13961399.

S6. D. Jacquemin, V. Wathelet, E. A. Perpete, C. Adamo, J. Chem. Theory Comput. 2009, 5, $2420-2435$.

S7. Hartlieb, K. J.; Liu, W.-G.; Fahrenbach, A. C.; Blackburn, A. K.; Frasconi, M.; Hafezi, N.; Dey, S. K.; Sarjeant, A. A.; Stern, C. L.; Goddard, W. A.; Stoddart, J. F., Chem. Eur. J. 2016, 22 (8), 2736-2745. 
S6. Shao, Y.; Gan, Z.; Epifanovsky, E.; Gilbert, A. T. B.; Wormit, M.; Kussmann, J.; Lange, A. W.; Behn, A.; Deng, J.; Feng, X.; Ghosh, D.; Goldey, M.; Horn, P. R.; Jacobson, L. D.; Kaliman, I.; Khaliullin, R. Z.; Kuś, T.; Landau, A.; Liu, J.; Proynov, E. I.; Rhee, Y. M.; Richard, R. M.; Rohrdanz, M. A.; Steele, R. P.; Sundstrom, E. J.; Woodcock, H. L.; Zimmerman, P. M.; Zuev, D.; Albrecht, B.; Alguire, E.; Austin, B.; Beran, G. J. O.; Bernard, Y. A.; Berquist, E.; Brandhorst, K.; Bravaya, K. B.; Brown, S. T.; Casanova, D.; Chang, C.M.; Chen, Y.; Chien, S. H.; Closser, K. D.; Crittenden, D. L.; Diedenhofen, M.; DiStasio, R. A.; Do, H.; Dutoi, A. D.; Edgar, R. G.; Fatehi, S.; Fusti-Molnar, L.; Ghysels, A.; GolubevaZadorozhnaya, A.; Gomes, J.; Hanson-Heine, M. W. D.; Harbach, P. H. P.; Hauser, A. W.; Hohenstein, E. G.; Holden, Z. C.; Jagau, T.-C.; Ji, H.; Kaduk, B.; Khistyaev, K.; Kim, J.; Kim, J.; King, R. A.; Klunzinger, P.; Kosenkov, D.; Kowalczyk, T.; Krauter, C. M.; Lao, K. U.; Laurent, A. D.; Lawler, K. V.; Levchenko, S. V.; Lin, C. Y.; Liu, F.; Livshits, E.; Lochan, R. C.; Luenser, A.; Manohar, P.; Manzer, S. F.; Mao, S.-P.; Mardirossian, N.; Marenich, A. V.; Maurer, S. A.; Mayhall, N. J.; Neuscamman, E.; Oana, C. M.; OlivaresAmaya, R.; O’Neill, D. P.; Parkhill, J. A.; Perrine, T. M.; Peverati, R.; Prociuk, A.; Rehn, D. R.; Rosta, E.; Russ, N. J.; Sharada, S. M.; Sharma, S.; Small, D. W.; Sodt, A.; Stein, T.; Stück, D.; Su, Y.-C.; Thom, A. J. W.; Tsuchimochi, T.; Vanovschi, V.; Vogt, L.; Vydrov, O.; Wang, T.; Watson, M. A.; Wenzel, J.; White, A.; Williams, C. F.; Yang, J.; Yeganeh, S.; Yost, S. R.; You, Z.-Q.; Zhang, I. Y.; Zhang, X.; Zhao, Y.; Brooks, B. R.; Chan, G. K. L.; Chipman, D. M.; Cramer, C. J.; Goddard, W. A.; Gordon, M. S.; Hehre, W. J.; Klamt, A.; Schaefer, H. F.; Schmidt, M. W.; Sherrill, C. D.; Truhlar, D. G.; Warshel, A.; Xu, X.; Aspuru-Guzik, A.; Baer, R.; Bell, A. T.; Besley, N. A.; Chai, J.-D.; Dreuw, A.; Dunietz, B. D.; Furlani, T. R.; Gwaltney, S. R.; Hsu, C.-P.; Jung, Y.; Kong, J.; Lambrecht, D. S.; Liang, W.; Ochsenfeld, C.; Rassolov, V. A.; Slipchenko, L. V.; Subotnik, J. E.; Van Voorhis, T.; Herbert, J. M.; Krylov, A. I.; Gill, P. M. W.; Head-Gordon, M., Mol. Phys. 2015, 113, 184215. 